\title{
Studies on the Photochemical Rearrangements of Enantiomerically Pure, Polysubstituted and Variously Annulated Bicyclo[2.2.2]octenones
}

\author{
Qiao Yan, Benoit Bolte, Yuhua Bai, Martin G. Banwell,* Anthony C. Willis \\ and Paul D. Carr
}

Research School of Chemistry, Institute of Advanced Studies, The Australian National University, Canberra, ACT 2601, Australia

A series of enantiomerically pure bicyclo[2.2.2]octenones, including the lactoneannulated system 26, has been prepared by engaging derivatives of an enzymaticallyderived and homochiral cis-1,2-dihydrocatechol in inter- or intra-molecular DielsAlder reactions. Systems such as $\mathbf{2 6}$ readily participate in photochemically promoted oxa-di- $\pi$-methane rearrangement or 1,3-acyl migration processes to give products such as diquinane $\mathbf{3 4}$ or mixtures of cyclobutanone $\mathbf{3 6}$ and cyclopropane $\mathbf{3 8}$, respectively. 


\section{INTRODUCTION}

Bicyclo[2.2.2] octenones including the parent system 1 (Scheme 1) are excellent substrates for certain photochemically promoted rearrangement reactions. ${ }^{1}$ Specifically, on irradiation in the presence of photosensitizers such as acetophenone they participate oxa-di- $\pi$-methane rearrangements and so affording, via a triplet pathway, cyclopropannulated diquinanes such as $\mathbf{2}$. In contrast, on direct irradiation they engage, now via a singlet pathway, in a 1,3-acyl migration reaction (Givens rearrangement) to give bicyclo[4.2.0]oct-4-en-7-ones such as 3. Upon sustained irradiation photoproduct $\mathbf{3}$ and many of its derivatives can undergo decarbonylation to give the corresponding $\Delta^{2}$-norcarene, e.g. 4.

Scheme 1: The oxa-di- $\pi$-methane, 1,3-acyl migration and photodecarbonylation reactions of the parent bicyclo[2.2.2] octenone (1).

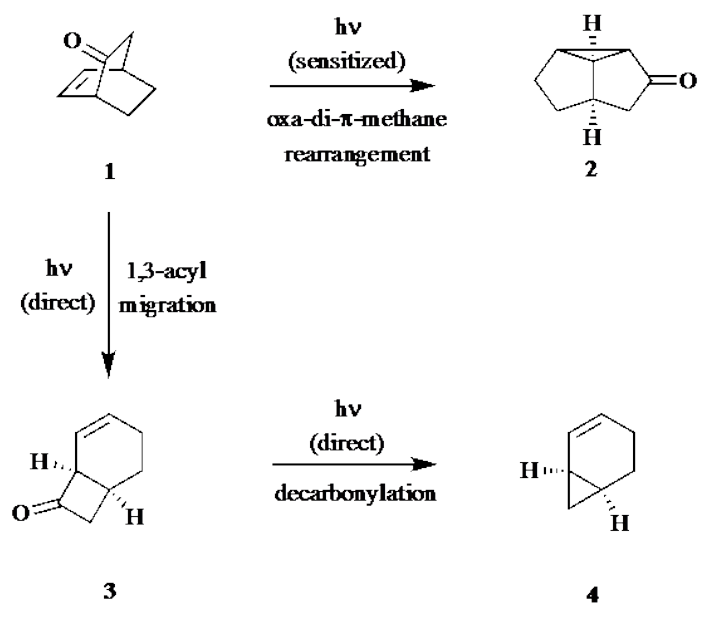

The capacity to effectively exploit these valuable transformations is contingent on having ready access to bicyclo[2.2.2]octenones, especially enantiomerically pure ones. While various methods are available for the synthesis of such systems, ${ }^{2}$ a particularly useful approach involves the facially-selective intermolecular 
cycloaddition of dienophiles to enantiomerically pure cis-1,2-dihydrocatechols such as 5 (Figure 1) that are readily obtained through the whole-cell biotransformation of the corresponding arene (toluene in the case of compound 5). ${ }^{3}$ Indeed, we have exploited such adducts in the photochemically-mediated total synthesis of a range of sesquiterpenoid natural products including various triquinanes, ${ }^{4}$ protoilludanes ${ }^{5}$ and the structure assigned to a sterpurene. ${ }^{6}$ In seeking to extend such studies we became interested in establishing the degree to which the bicyclo[2.2.2]octenone framework could be substituted and/or annulated and continue to engage in the above-mentioned photochemical processes. Herein we detail research that serves to emphasize the remarkable extent to which these processes can be applied in a reliable fashion.

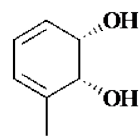

5

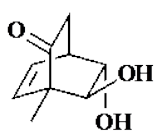

6

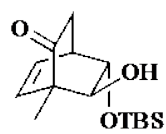

7

Figure 1: Homochiral cis-1,2-dihydrocatechol 5 and the derived bicyclo[2.2.2]octenone-based photosubstrates $\mathbf{6}$ and $\mathbf{7 .}$

In connection with our recently reported studies ${ }^{7}$ on the synthesis of the sorbicillinoid-derived isolate rezishanone $\mathrm{C}$ we prepared, ${ }^{7}$ using intermolecular DielsAlder cycloaddition processes, the oxygenated bicyclo[2.2.2]octenones $\mathbf{6}$ and $\mathbf{7}$ in gram quantities from cis-1,2-dihydrocatechol 5. Accordingly, compounds 6 and 7 became the substrates used in the opening stages of the present work. 


\section{RESULTS AND DISCUSION}

In keeping with expectations, independent irradiation (using a medium pressure mercury lamp) of an acetone solution of each of compounds $\mathbf{6}$ and $\mathbf{7}$ held in a roundbottomed flask made from borosilicate glass and containing ca. 1.4 mole equivalents of acetophenone (Scheme 2) afforded the anticipated oxa-di- $\pi$-methane rearrangement products 8 ( $87 \%$ or $98 \%$ brsm) and 9 (90\% or $99 \%$ brsm), respectively. All of the spectral data acquired on compounds $\mathbf{8}$ and $\mathbf{9}$ were entirely consistent with the assigned structures but final confirmation of these followed from single-crystal Xray analyses (see the Experimental Section and Supporting Information - SI - for details).

Scheme 2: Photochemical behaviors of bicyclo[2.2.2]octenones 6 and 7 under either direct irradiation or photosensitized conditions.

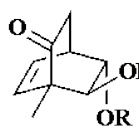

$$
6 \mathrm{R}=\mathrm{H}
$$

$7 \mathrm{R}=\mathrm{TBS}$

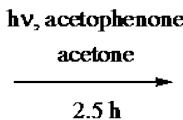

$2.5 \mathrm{~h}$

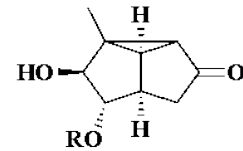

$\mathbf{8} \mathrm{R}=\mathbf{H}(\mathbf{8 7} \%)$

$9 \mathrm{R}=$ TBS $(90 \%)$

\section{(a) hv, $\mathrm{CH}_{2} \mathrm{Cl}_{2}$}

0.67 to $0.83 \mathrm{~h}$

(b) hv, $\mathrm{CH}_{2} \mathrm{Cl}$

$5 \mathrm{~h}$
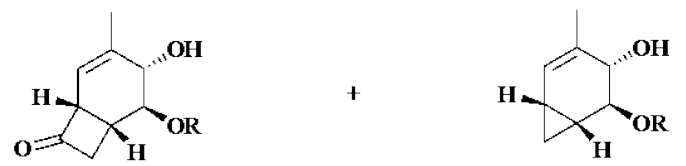

$10 \mathrm{R}=\mathrm{H}(70 \%$ after $0.83 \mathrm{~h})$

$11 \mathrm{R}=$ TBS $(67 \%$ after $0.67 \mathrm{~h})$

$12 \mathrm{R}=\mathrm{H}(32 \%$ after $5 \mathrm{~h})$

$13 \mathrm{R}=$ TBS (33\% after $5 \mathrm{~h}$ )

When dichloromethane solutions of substrates 6 and 7 were subjected to direct irradiation for brief periods (less than one hour) with the same type of lamp then the anticipated Givens rearrangement products 10 (70\% or 84\% brsm) and 11 (67\%), 
respectively, were obtained. Upon sustained irradiation (5 hours) of the same substrates then significant quantities of corresponding decarbonylated systems $\mathbf{1 2}$ (32\%) and 13 (33\%) were obtained along with reduced amounts of the corresponding and chromatographically separable cyclobutenones. Once again, all the spectroscopic data acquired on compounds $\mathbf{1 0}$ to $\mathbf{1 3}$ were in according with the assigned structures but those of the first and the last of these were confirmed by single-crystal X-ray analysis.

Establishing the impact of various modes of ring-fusion on the capacity of bicyclo[2.2.2] octenones to engage in oxa-di- $\pi$-methane and 1,3-acyl migration reactions was another topic of interest. So, building upon our earlier studies ${ }^{8}$ on the assembly of the relevant frameworks through the intramolecular Diels-Alder (IMDA) reactions of crotonate esters derived from cis-1,2-dihydrocatechols, compound $\mathbf{5}$ was converted (Scheme 3) into the diesters 14 (81\%) and 15 (74\%) under previously established ${ }^{8}$ conditions. When each of compounds $\mathbf{1 4}$ and $\mathbf{1 5}$ was heated in refluxing toluene these engaged in the two possible modes of cycloaddition and thus giving rise to the corresponding pair of adducts, viz. lactones 16 (40\% from 14), 17 (41\% from 15), 18 (26\% from 14) and 19 (12\% from 15) that could be separated from one another by conventional chromatographic means. The remaining ester residues within each of adducts 18 and 19 were cleaved by standard methods and the resulting alcohols 20 (74\%) and 21 (76\%) oxidized to the corresponding ketones 22 (86\%) and 23 (82\%), respectively, using the Dess-Martin periodinane. ${ }^{9}$ Similarly, esters $\mathbf{1 6}$ and $\mathbf{1 7}$ were cleaved using potassium carbonate in methanol and the product alcohols 24 (79\%) and 25 (94\%), respectively, oxidized to the corresponding ketones, namely compounds 26 (94\%) and 27 (78\%). Single-crystal X-ray analyses were conducted on 
compounds 23 and 27, details of which are presented in the Experimental Section and the SI.

Scheme 3: Synthesis of lactone-annulated bicyclo[2.2.2]octenones 22, 23, 26 and 27

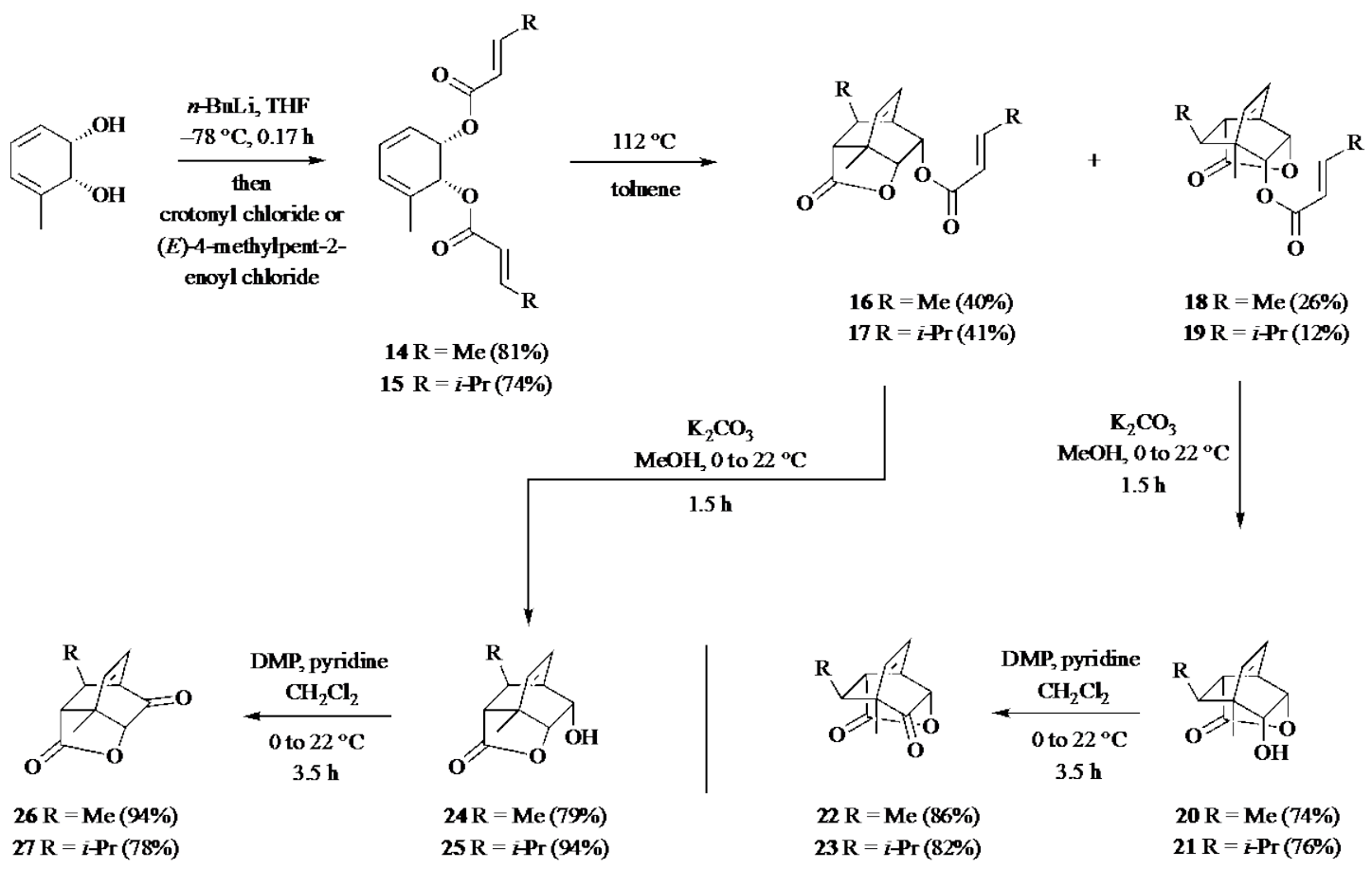

The oxa-di- $\pi$-methane rearrangement of the lactone-annulated bicyclo[2.2.2]octenones 22 and 23 took place readily when acetone solutions of each of these was irradiated in the presence of acetophenone (Scheme 4). By such means the cyclopropane-annulated oxa-triquinanes 28 (40\%) and 29 (43\%) were obtained. In contrast, direct irradiation of substrates 22 and 23 led to the corresponding mixtures of cyclobutanones $\mathbf{3 0}$ (up to 75\%) and $\mathbf{3 1}$ (up to 73\%), respectively, as well as their decarbonylated counterparts 32 (up to $37 \%$ ) and 33 (up to 52\%), respectively. 
Scheme 4: Photochemical behaviors of bicyclo[2.2.2]octenones 22 and 23 under either direct irradiation or sensitized conditions.

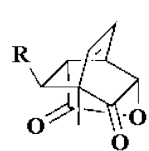

$$
22 \mathrm{R}=\mathrm{Me}
$$$$
23 \mathrm{R}=\boldsymbol{i} \mathbf{P r}
$$

hv, acetophenone

acetone

0.67 to $0.83 \mathrm{~h}$$$
\text { ( }
$$

$$
28 \mathrm{R}=\mathrm{Me}(40 \%)
$$$$
29 \mathrm{R}=\overline{-} \operatorname{Pr}(43 \%)
$$

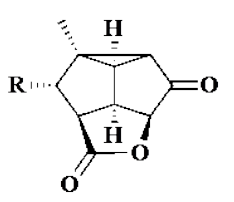

$29 \mathrm{R}=\operatorname{Tr}(43 \%)$

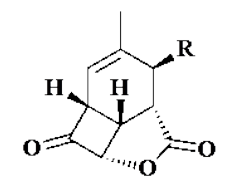

$30 \mathrm{R}=\mathrm{Me}$ (75\% after $0.5 \mathrm{~h})$ $31 \mathrm{R}=\boldsymbol{i} \operatorname{Pr}(73 \%$ after $0.5 \mathrm{~h})$

The structures of compounds $\mathbf{3 0}$ and $\mathbf{3 2}$ were confirmed by single-crystal X-ray analyses.

Equivalent studies on the photochemical behaviors of the isomeric lactone annulated bicyclo[2.2.2]octenones 26 and 27 (Scheme 5) led to analogous outcomes. Specifically, the anticipated oxa-triquinanes 34 (45\%) and 35 (49\%) were obtained on photosensitized irradiation of these substrates while direct irradiation afforded mixtures of cyclobutanones 36 (up to $70 \%$ ) and 37 (up to $71 \%$ ) as well as their decarbonylated congeners 38 (up to $35 \%$ ) and 39 (up to 40\%), respectively. The structures of compounds 34, 35, 36 and 38 were each confirmed by single-crystal Xray analyses. 
Scheme 5: Photochemical behaviors of bicyclo[2.2.2]octenones 26 and 27 under either direct irradiation or sensitized conditions.

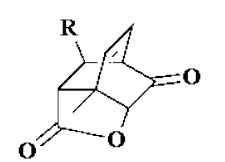

$26 \mathrm{R}=\mathrm{Me}$

$27 \mathrm{R}=i \mathbf{P r}$

(a) hv, $\mathrm{CH}_{2} \mathrm{Cl}_{2}, 0.55$ to $0.58 \mathrm{~h}$

(b) hv, $\mathrm{CH}_{2} \mathrm{Cl}_{2}, 3.1 \mathrm{~h}$
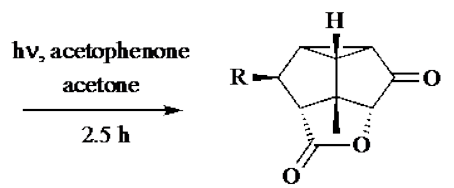

$34 \mathrm{R}=\mathrm{Me}(45 \%)$

$35 \mathrm{R}=\bar{i} \operatorname{Pr}(49 \%)$

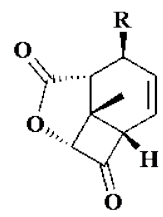

$36 \mathrm{R}=\mathrm{Me}(70 \%$ after $0.58 \mathrm{~h})$

$37 \mathrm{R}=i-\operatorname{Pr}(71 \%$ after $0.55 \mathrm{~h})$

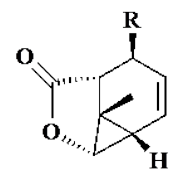

$38 \mathrm{R}=$ Me (35\% after $3.1 \mathrm{~h})$

$39 \mathrm{R}=\bar{t} \operatorname{Pr}(40 \%$ after $3.1 \mathrm{~h})$

It is worth noting that the photoproducts shown in Scheme 5 are pseudo-enantiomers of their counterparts shown in Scheme 4. Or, to put matters another way, and for the purposes of illustrating the point, if each of compounds 30 (Scheme 4) and $\mathbf{3 6}$ (Scheme 5) lacked the two methyl groups then they would be enantiomers. Accordingly, the protocols just described provide a means by which the single enantiomeric form of starting material $\mathbf{5}$ can be converted into either enantiomeric form of a range of relatively complex molecular frameworks.

In seeking to establish the effects of alternate modes of ring fusion and other substituents on the capacity of bicyclo[2.2.2]octenones to engage in photochemicallypromoted rearrangements, the behaviors of various polyhydro-4,7-ethanoindenone derivatives were explored. The routes shown in Scheme 6 were used to prepare these

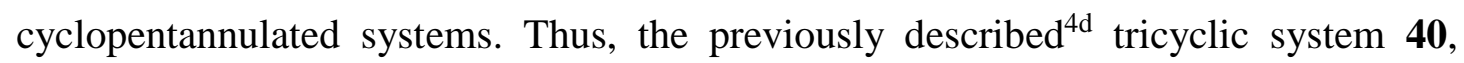


prepared by a reaction sequence involving an initial Diels-Alder cycloaddition reaction between 2-cyclopenten-1-one and the acetonide derived from cis-diol 5, was reduced with $\mathrm{LiAlH}_{4}$ and previously reported ${ }^{4 d, 5 b}$ alcohol 41 (39\%) obtained in pure form after chromatographic separation from it co-produced epimer. ${ }^{4 \mathrm{~d}, 5 \mathrm{~b}}$ Compound 41, the structure of which was confirmed by single-crystal X-ray analysis, was acetylated under standard conditions to give ester 42 (97\%) and the acetonide moiety associated with this last compound was hydrolyzed by treating a methanol/water solution of it with acidified AG-50W-X8 resin. The product diol 43 (97\%) was selectively oxidized using the sterically demanding oxammonium salt derived from $p$ $\mathrm{TsOH} \cdot \mathrm{H}_{2} \mathrm{O}$-promoted disproportionation of 4 -acetamido-TEMPO ${ }^{10}$ and thus producing acyloin 44 (97\%) that was also subject to single-crystal X-ray analysis. Compound 44 was converted into derivatives 45 (91\%), 46 (77\%), 47 (82\%) and 48 (93\%) by standard methods while samarium iodide-mediated deoxygenation of benzoate 48 also allowed for the preparation of bicyclo[2.2.2]octenone 49 (85\%). Once again, the structures of compounds 45,47 and 48 were confirmed by singlecrystal X-ray analyses.

On direct irradiation of dichloromethane solutions of each of compounds $\mathbf{4 5}$ to $\mathbf{4 9}$ they engaged in 1,3-acyl migration reactions to give the cyclobutanones $\mathbf{5 0}$ (43\% or 99\% brsm), 51 (27\% or 98\% brsm), 52 (18\% or 99\% brsm), 53 (17\% or quant. brsm) and 54 (23\% or $92 \%$ brsm), respectively. Irradiation of compounds $\mathbf{5 0}$ and $\mathbf{4 7}$ led to cyclopropanes 55 (98\%) and 56 (80\%), respectively. The structures of all of these photoproducts were determined through comprehensive spectroscopic analyses. In particular, the infra-red spectrum of each of cyclobutanones 50-54 exhibited a characteristic carbonyl absorption band in the range $1778-1792 \mathrm{~cm}^{-1}$ as well as a 
carbonyl carbon resonance above $\delta 200 \mathrm{ppm}$ in the corresponding ${ }^{13} \mathrm{C}$ NMR spectrum. The structure of compound 53 was confirmed by single-crystal X-ray analysis.

Scheme 6: Synthesis of cyclopentannulated bicyclo[2.2.2]octenones 45-49 and their photochemical behaviors under direct irradiation conditions

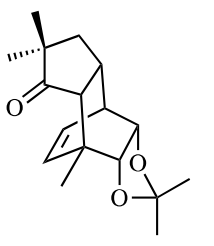

40

$$
\begin{gathered}
\underset{-30 \text { to } 22{ }^{\circ} \mathrm{C}}{\stackrel{\mathrm{LiAlH}_{4}, \mathrm{THF}}{\longrightarrow}} \\
\begin{array}{c}
6.5 \mathrm{~h}, 39 \%
\end{array}
\end{gathered}
$$

$$
\begin{gathered}
\stackrel{\left(\mathrm{H}^{+} \text {form }\right)}{\mathrm{MeOH} / \text { water }} \\
65^{\circ} \mathrm{C}, 67 \mathrm{~h} \\
97 \%
\end{gathered}
$$

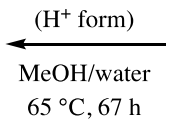

AG-50W-X8 resin

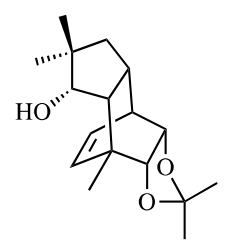

41

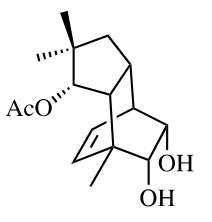

43

$$
\begin{gathered}
\mathrm{Ac}_{2} \mathrm{O}, \text { DMAP } \\
\text { pyridine } \\
0 \text { to } 22{ }^{\circ} \mathrm{C}, 4 \mathrm{~h} \\
97 \%
\end{gathered}
$$

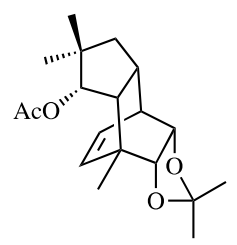

42

$\downarrow \begin{gathered}\text { 4-acetamido-TEMPO } \\ p \text {-TsOH} \cdot \mathrm{H}_{2} \mathrm{O} \\ \mathrm{CH}_{2} \mathrm{Cl}_{2}, 0^{\circ} \mathrm{C}, 2.5 \mathrm{~h}, 97 \%\end{gathered}$

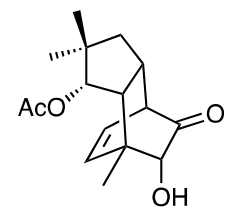

44

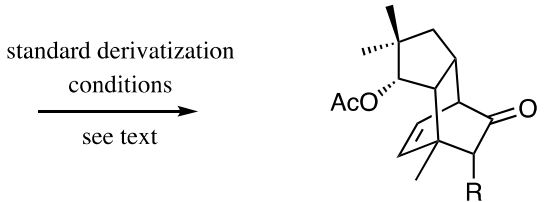<smiles>[R]C1[C@H]2C=C[C@@H]3CC(C)(C)C(OC(C)C)[C@]3(C)[C@]12C</smiles>

$\frac{\mathrm{hv}, \mathrm{CH}_{2} \mathrm{Cl}_{2}}{0.5-1.67 \mathrm{~h}}$

$\mathbf{5 5} \mathrm{R}=$ OAc $(98 \%)$

$56 \mathrm{R}=$ OTBS $(80 \%)$

$45 \mathrm{R}=\mathrm{OAc}(91 \%)$ $46 \mathrm{R}=\mathrm{OMOM}(77 \%)$ $47 \mathrm{R}=$ OTBS $(82 \%)$

$\mathrm{SmI}_{2}, \mathrm{THF} / \mathrm{methanol}-\mathbf{4 8} \mathrm{R}=\mathrm{OBz}(93 \%)$ $-78{ }^{\circ} \mathrm{C}, 1.25 \mathrm{~h} \longrightarrow 49 \mathrm{R}=\mathrm{H}(85 \%)$

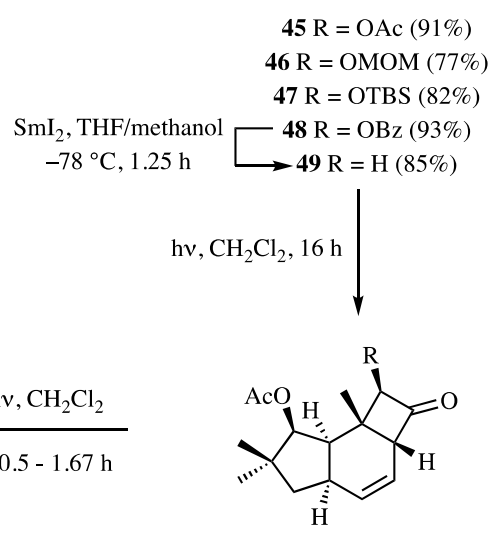

$\mathbf{5 0} \mathrm{R}=$ OAc $(43 \%$ or $99 \%$ brsm $)$ $51 \mathrm{R}=$ OMOM $(27 \%$ or $98 \%$ brsm $)$ $52 \mathrm{R}=$ OTBS $(18 \%$ or $99 \%$ brsm $)$ $53 \mathrm{R}=\mathrm{OBz}$ (17\% or quant. brsm) $54 \mathrm{R}=\mathrm{H}(23 \%$ or $92 \%$ brsm $)$ 
Given our extensive earlier studies ${ }^{4}$ on the oxa-di- $\pi$-methane rearrangements of compounds very closely related to compounds $\mathbf{4 5 - 4 9}$ we have not subjected these photosensitized irradiation but would fully expect them to behave in a similar manner and so leading the corresponding cyclopropannulated triquinanes.

In contrast to outcomes noted above (Scheme 6), when the mesylate 57 (Scheme 7), that was readily prepared in 56\% yield from acyloin 44 , was subject (as a solution in dichloromethane) to direct irradiation it engaged in an oxa-di- $\pi$-methane rearrangement reaction rather than a 1,3-acyl migration reaction and so affording the cyclopentannulated and stereochemically pure triquinane $\mathbf{5 8}$ in $48 \%$ yield (or $98 \%$ yield brsm). The configuration at the carbon bearing the mesyloxy group is tentatively assigned as illustrated although the alternate one cannot be discounted as a result of the intervention of a photo-epimerization process. ${ }^{4 \mathrm{~b}-\mathrm{d}}$ The precise origins of this seemingly anomalous behavior of sulfonate ester $\mathbf{5 7}$ remain unclear at the present time and serve to highlight the very limited understanding of the photochemical properties of such systems. ${ }^{11}$ These matters are the subject of ongoing $\rightarrow \rightarrow$ studies in our laboratories. 
Scheme 7: Synthesis and anomalous photochemical behavior of the mesyloxysubstituted bicyclo[2.2.2]octenone 57.

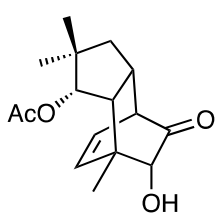

44

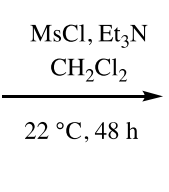

${ }^{\circ} \mathrm{C}, 48 \mathrm{~h}$

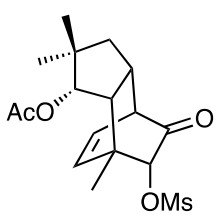

$57(56 \%)$
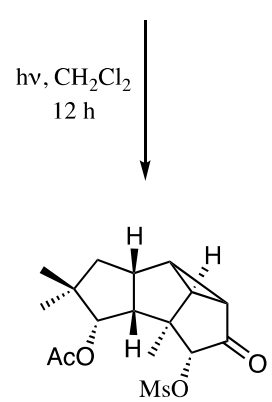

( $48 \%$ or $98 \%$ brsm)

\section{CONCLUSIONS}

The studies reported here have established that a wide range of extensively substituted and variously annulated bicyclo[2.2.2]octenones are capable of engaging in either oxa-di- $\pi$-methane or 1,3-acyl migration reaction depending upon the mode of photoactivation (sensitized vs direct irradiation). The 1,3-acyl migration reactions leading to the isomeric cyclobutanones can be followed by rapid photodecarbonylation processes and so co-producing the corresponding (but normally readily separable) cyclopropanes. A noteworthy feature of the photochemical behaviors of the annulated bicyclo[2.2.2]octenones studied here is that the mode of ring fusion (annulation) can have a significant impact on the rates of both types of processes. So, for example, the lactone-annulated systems 22, 23, 26 and 27 engage in more rapid 1,3-acyl migration reactions than their cyclopentannulated counterparts $\mathbf{4 5 - 4 9}$. The same could be said of the corresponding oxa-di- $\pi$-methane process, especially when the outcomes of our 
earlier studies ${ }^{4}$ are taken into account. That is to say, the lactone annulated compounds just mentioned also participate in more rapid triplet-sensitized rearrangement reactions than their cyclopentannulated counterparts. The origins of these variations are the subject of ongoing studies, the outcomes of which will be reported in due course.

\section{EXPERIMENTAL SECTION}

\section{General Experimental Procedures}

Unless otherwise specified, proton $\left({ }^{1} \mathrm{H}\right)$ and carbon $\left({ }^{13} \mathrm{C}\right)$ NMR spectra were recorded at $18{ }^{\circ} \mathrm{C}$ in base-filtered $\mathrm{CDCl}_{3}$ on a spectrometer operating at $400 \mathrm{MHz}$ for proton and $100 \mathrm{MHz}$ for carbon nuclei. ${ }^{1} \mathrm{H}$ NMR data are recorded as follows: chemical shift

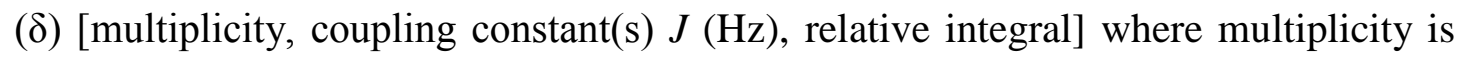
defined as $\mathrm{s}=$ singlet; $\mathrm{d}=$ doublet; $\mathrm{t}=$ triplet; $\mathrm{q}=$ quartet; $\mathrm{m}=$ multiplet or combinations of the above. In relevant cases, the signal due to residual $\mathrm{CHCl}_{3}$ appearing at $\delta_{\mathrm{H}} 7.26$ and the central resonance of the $\mathrm{CDCl}_{3}$ "triplet" appearing at $\delta_{\mathrm{C}}$ 77.0 were used to reference ${ }^{1} \mathrm{H}$ and ${ }^{13} \mathrm{C}$ NMR spectra, respectively. Samples were analyzed by infrared spectroscopy $\left(v_{\max }\right)$ as thin films on $\mathrm{KBr}$ plates. Optical rotations were recorded using the sodium D-line (589 nm) in a cell with a path length of $1 \mathrm{dm}$, at the concentrations indicated and in the specified solvent at $22{ }^{\circ} \mathrm{C}$. Specific rotations were then calculated in the usual manner. Low- and high-resolution electron impact (EI) mass spectra were recorded on a double focusing, triple sector machine. Lowand high-resolution ESI mass spectra were recorded on a triple-quadrupole mass spectrometer operating in positive ion mode. Melting points are uncorrected. Analytical thin layer chromatography (TLC) was performed on aluminum-backed 0.2 mm thick silica gel $60 \mathrm{~F}_{254}$ plates. Eluted plates were visualized using a $254 \mathrm{~nm}$ UV 
lamp and/or by treatment with a suitable dip followed by heating. These dips included phosphomolybdic acid/ceric sulfate/sulfuric acid (conc.)/water (37.5 g : $7.5 \mathrm{~g}: 37.5 \mathrm{~g}$ : $720 \mathrm{~mL}$ ), potassium permanganate/potassium carbonate/5\% sodium hydroxide aqueous solution/water (3 g : $20 \mathrm{~g}: 5 \mathrm{~mL}: 300 \mathrm{~mL}$ ), and $p$-anisaldehyde or vanillin/sulfuric acid (conc.)/ethanol (15 g : $2.5 \mathrm{~mL}: 250 \mathrm{~mL}$ ). Flash chromatographic separations were carried out following protocols defined by Still et al. ${ }^{12}$ with silica gel $60(40-63 \mu \mathrm{m})$ as the stationary phase and using the AR- or HPLC-grade solvents indicated. The melting points of solids purified by such means were recorded directly (ie after they had crystallized from the concentrated chromatographic fractions). Starting materials, reagents, drying agents, and other inorganic salts were generally commercially available and were used as supplied. Tetrahydrofuran (THF), methanol and dichloromethane were dried using a solvent purification system that is based upon a technology originally described by Grubbs et al. ${ }^{13}$ Where necessary, reactions were performed under a nitrogen atmosphere.

\section{Specific Chemical Transformations}

\section{(2a'R,3S,4S,4aR)-3,4-Dihydroxy-2b-methylhexahydrocyclopropa[cd]pentalen-}

2(1H)-one (8). A deoxygenated solution of compound $\mathbf{6}^{7}$ (94 mg, $0.56 \mathrm{mmol}$ ) and acetophenone $(90 \mu \mathrm{L}, 0.77 \mathrm{mmol})$ in acetone $(100 \mathrm{~mL})$ maintained under nitrogen was subjected to irradiation with a Hanovia 450W medium pressure mercury-vapor lamp for $2.5 \mathrm{~h}$ then cooled and concentrated under reduced pressure to give a yellow oil. This material was subjected to flash column chromatography (silica, 1:1 $\rightarrow$ 1:0 v/v ethyl acetate/40-60 petroleum ether gradient elution) to afford two fractions, A and B.

Concentration of fraction A ( $R_{\mathrm{f}}=0.3$ in ethyl acetate) afforded the starting compound 6 (10 mg, 11\% recovery) as a colorless, crystalline solid that was identical, 
in all respects, with an authentic sample.

Concentration of fraction B ( $R_{\mathrm{f}}=0.1$ in ethyl acetate) afforded compound 8 (82 mg, $87 \%$ or $98 \%$ brsm) as a white, crystalline solid, $\mathrm{mp}=169-171{ }^{\circ} \mathrm{C}:[\alpha]_{\mathrm{D}}=$ +71.4 (c 1.0, methanol). ${ }^{1} \mathrm{H}$ NMR (400 MHz, CD $\left.{ }_{3} \mathrm{OD}\right) \delta 4.15(\mathrm{~d}, J=2.6 \mathrm{~Hz}, 1 \mathrm{H})$, $3.74(\mathrm{~s}, 1 \mathrm{H}), 2.81$ (t, $J=5.0 \mathrm{~Hz}, 1 \mathrm{H}), 2.72(\mathrm{~m}, 1 \mathrm{H}), 2.42$ (m, 1H), 2.08 (m, 1H), 1.82 (d, $J=5.0 \mathrm{~Hz}, 1 \mathrm{H}$ ), 1.37 (s, 3H) (signals due to $\mathrm{O}-\mathrm{H}$ group protons not observed); ${ }^{13} \mathrm{C}$ NMR (100 MHz, CD 3 OD) $\delta$ 217.3, 88.2, 86.3, 47.8, 47.0, 46.3(0), 46.2(8), 44.2, 21.5; IR $v_{\max }$ 3356, 2962, 2904, 1706, 1693, 1290, 1040, $898 \mathrm{~cm}^{-1}$; MS (ESI, +ve) m/z 191 $\left[(\mathrm{M}+\mathrm{Na})^{+}, 100 \%\right]$; HRMS $(\mathrm{M}+\mathrm{Na})^{+}$calcd for $\mathrm{C}_{9} \mathrm{H}_{12} \mathrm{O}_{3}$ 191.0684, found 191.0685 .

(2a'R,3S,4S,4aR)-4-((tert-Butyldimethylsilyl)oxy)-3-hydroxy-2bmethylhexahydro -cyclopropa[cd]pentalen-2(1H)-one (9). A deoxygenated solution of compound $7^{7}$ (158 $\mathrm{mg}, 0.56 \mathrm{mmol})$ and acetophenone $(90 \mu \mathrm{L}, 0.77 \mathrm{mmol})$ in acetone $(100 \mathrm{~mL})$ maintained under nitrogen was subjected to irradiation with a Hanovia 450W medium pressure mercury-vapor lamp for $2.5 \mathrm{~h}$. The cooled reaction mixture was concentrated under reduced pressure to give a clear, yellow oil and this was subjected to flash column chromatography (silica, 1:9 $\rightarrow$ 3:7 v/v ethyl acetate/40-60 petroleum ether gradient elution) to afford two fractions, A and B.

Concentration of fraction A $\left(R_{\mathrm{f}}=0.4\right.$ in 3:7 v/v ethyl acetate $/ 40-60$ petroleum ether) afforded compound 7 (13 mg, 8\% recovery) as a colorless crystalline solid that was identical, in all respects, with an authentic sample.

Concentration of fraction B $\left(R_{\mathrm{f}}=0.2\right.$ in 3:7 v/v ethyl acetate/40-60 petroleum ether) afforded compound 9 (143 mg, 90\% or 99\% brsm) as a white, crystalline solid, $\mathrm{mp}=99-100{ }^{\circ} \mathrm{C}:[\alpha]_{\mathrm{D}}=+41.8\left({ }^{c} 1.0, \mathrm{CHCl}_{3}\right) .{ }^{1} \mathrm{H} \mathrm{NMR}\left(800 \mathrm{MHz}, \mathrm{CDCl}_{3}\right) \delta 4.20(\mathrm{~d}$, $J=2.5 \mathrm{~Hz}, 1 \mathrm{H}), 3.81(\mathrm{~s}, 1 \mathrm{H}), 2.76(\mathrm{t}, J=5.1 \mathrm{~Hz}, 1 \mathrm{H}), 2.66(\mathrm{~m}, 1 \mathrm{H}), 2.38(\mathrm{~m}, 1 \mathrm{H})$, $2.13(\mathrm{~d}, J=17.9 \mathrm{~Hz}, 1 \mathrm{H}), 1.86$ (d, $J=5.0 \mathrm{~Hz}, 1 \mathrm{H}), 1.79$ (broad s, 1H), 1.35 (s, 3H), 
0.90 (s, 9H), 0.08 (s, 6H); ${ }^{13} \mathrm{C}$ NMR (200 MHz, $\left.\mathrm{CDCl}_{3}\right) \delta$ 213.3, 86.8, 85.8, 45.3, 45.0(4), 44.9(8), 44.4, 42.2, 24.9, 20.4, 17.2, -5.6; IR $v_{\max }$ 3351, 2957, 2929, 2886, 2857, 1719, 1254, 1092, 867, 836, $771 \mathrm{~cm}^{-1}$; MS (ESI, +ve) m/z $305\left[(\mathrm{M}+\mathrm{Na})^{+}\right.$, 100\%], $283\left[(\mathrm{M}+\mathrm{H})^{+}, 30\right]$; HRMS $(\mathrm{M}+\mathrm{Na})^{+}$calcd for $\mathrm{C}_{15} \mathrm{H}_{26} \mathrm{O}_{3} \mathrm{Si} 305.1549$, found 305.1548.

$(1 R, 2 S, 3 S, 6 R)-2,3-D i h y d r o x y-4-m e t h y l b i c y c l o[4.2 .0]$ oct-4-en-7-one $\quad(10) . \quad$ A magnetically stirred and deoxygenated solution of compound 6 (100 mg, $0.59 \mathrm{mmol}$ ) in dichloromethane $(100 \mathrm{~mL})$ maintained under nitrogen was subjected to direct irradiation with a Hanovia 450W medium pressure mercury-vapor lamp for $0.83 \mathrm{~h}$. The cooled reaction mixture was concentrated under reduced pressure to give a yellow solid that was subjected to flash column chromatography (silica, $2: 3 \rightarrow 1: 0 \mathrm{v} / \mathrm{v}$ ethyl acetate/40-60 petroleum ether gradient elution) to afford two fractions, A and B.

Concentration of fraction A ( $R_{\mathrm{f}}=0.4$ in ethyl acetate) afforded compound $\mathbf{1 0}$ (70 mg, $70 \%$ or $84 \%$ brsm) as a white, crystalline solid, $\mathrm{mp}=83-85{ }^{\circ} \mathrm{C}:[\alpha]_{\mathrm{D}}=$ -234.4 (c 1.0, MeOH). ${ }^{1} \mathrm{H}$ NMR (400 MHz, CD $\left.{ }_{3} \mathrm{OD}\right) \delta 5.40$ (m, 1H), 3.96 (d, $J=6.9$ Hz, 1H), 3.88 (m, 1H), 3.47 (t, $J=7.5$ Hz, 1H), 3.24 (m, 1H), 2.98 (m, 1H), 2.57 (m, 1H), 1.81 (s, 3H) (signals due to $\mathrm{O}-\mathrm{H}$ group protons not observed); ${ }^{13} \mathrm{C}$ NMR (100 $\left.\mathrm{MHz}, \mathrm{CD}_{3} \mathrm{OD}\right) \delta 209.0,140.2,116.7,76.2,73.6,61.0,50.1,30.2,20.0$; IR $v_{\max } 3391$, 2882, 1769, 1066, 1047, 1014, $884 \mathrm{~cm}^{-1}$; MS (ESI, +ve) m/z $223\left[(\mathrm{M}+\mathrm{MeOH}+\mathrm{Na})^{+}\right.$, 40\%], $191\left[(\mathrm{M}+\mathrm{Na})^{+}, 100\right]$; HRMS $(\mathrm{M}+\mathrm{Na})^{+}$calcd for $\mathrm{C}_{9} \mathrm{H}_{12} \mathrm{O}_{3}$ 191.0684, found 191.0684

Concentration of fraction B ( $R_{\mathrm{f}}=0.3$ in ethyl acetate) afforded compound 6 (14 mg, 14\% recovery) as a colorless, crystalline solid that was identical, in all respects, with an authentic sample. 
(1R,2S,3S,6R)-2-((tert-Butyldimethylsilyl)oxy)-3-hydroxy-4-methylbicyclo[4.2.0]-

oct-4-en-7-one (11). The magnetically stirred and deoxygenated solution of compound 7 (100 mg, $0.35 \mathrm{mmol})$ in dichloromethane $(100 \mathrm{~mL})$ maintained under nitrogen was subjected to direct irradiation with a Hanovia 450W medium pressure mercury-vapor lamp for $0.67 \mathrm{~h}$. The cooled reaction mixture was concentrated under reduced pressure to give a pale-yellow oil. This was subjected to flash column chromatography (silica, 1:9 v/v ethyl acetate/40-60 petroleum ether elution) to give, after concentration of the appropriate fractions $\left(R_{\mathrm{f}}=0.6\right.$ in $3: 7 \mathrm{v} / \mathrm{v}$ ethyl acetate/40-60 petroleum ether), compound 11 (67 mg, 67\%) as a white, crystalline solid, $\mathrm{mp}=67$ $69{ }^{\circ} \mathrm{C}:[\alpha]_{\mathrm{D}}=-139.6\left(\right.$ c 1.0, $\left.\mathrm{CHCl}_{3}\right) .{ }^{1} \mathrm{H}$ NMR (400 MHz, $\left.\mathrm{CDCl}_{3}\right) \delta 5.38(\mathrm{~m}, 1 \mathrm{H})$, 4.04 (d, $J=7.0 \mathrm{~Hz}, 1 \mathrm{H}), 3.86$ (m, 1H), 3.57 (t, $J=7.7 \mathrm{~Hz}, 1 \mathrm{H}), 3.24$ (m, 1H), 2.94 (m, 1H), 2.57 (m, 1H), 2.18 (broad s, 1H), 1.82 (s, 3H), 0.90 (s, 9H), 0.13 (s, 3H), 0.10 (s, 3H); ${ }^{13} \mathrm{C}$ NMR (100 MHz, $\left.\mathrm{CDCl}_{3}\right) \delta 207.0,138.4,115.1,77.9,73.4,60.6$, 50.2, 29.9, 25.9, 19.1, 18.3, -3.8, -4.2; IR $v_{\max } 3499,2954,2930,2857,1779,1126$, 1087, $836 \mathrm{~cm}^{-1}$; MS (ESI, +ve) m/z $337\left[(\mathrm{M}+\mathrm{MeOH}+\mathrm{Na})^{+}, 30 \%\right], 305\left[(\mathrm{M}+\mathrm{Na})^{+}\right.$, 100]; HRMS (M+Na) ${ }^{+}$calcd for $\mathrm{C}_{15} \mathrm{H}_{26} \mathrm{O}_{3} \mathrm{Si} 305.1549$, found 305.1554.

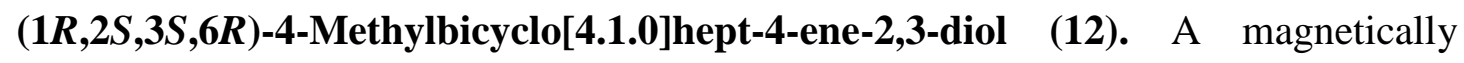
stirred and deoxygenated solution of compound 6 (100 mg, $0.59 \mathrm{mmol})$ in dichloromethane (100 mL) maintained under a nitrogen atmosphere was subjected to direct irradiation with a Hanovia 450W medium pressure mercury-vapor lamp for $5 \mathrm{~h}$. The cooled reaction mixture was concentrated under reduced pressure to give a brown oil that was subjected to flash column chromatography (silica, 1:4 $\rightarrow 1: 1 \mathrm{v} / \mathrm{v}$ ethyl acetate/40-60 petroleum ether gradient elution) to afford two fractions, A and B.

Concentration of fraction A ( $R_{\mathrm{f}}=0.6$ in ethyl acetate) afforded compound 12 (27 mg, 32\%) as a pale-yellow oil, $[\alpha]_{\mathrm{D}}=+93.2\left(\mathrm{c} 0.8, \mathrm{CHCl}_{3}\right) .{ }^{1} \mathrm{H} \mathrm{NMR}(400 \mathrm{MHz}$, 
$\left.\mathrm{CDCl}_{3}\right) \delta 6.00$ (m, 1H), 4.04-3.99 (complex m, 2H), 1.92 (broad s, 2H), 1.76 (s, 3H), 1.48-1.35 (complex m, 2H), 1.03 (m, 1H), 0.64 (m, 1H); ${ }^{13} \mathrm{C}$ NMR (100 MHz, $\left.\mathrm{CDCl}_{3}\right)$ $\delta$ 130.3, 127.8, 73.3, 70.1, 21.4, 19.6, 18.7, 11.0; IR $v_{\max } 3367,2920,1445,1260$, 1048, 1001, $724 \mathrm{~cm}^{-1}$; MS (EI, $\left.70 \mathrm{eV}\right) \mathrm{m} / \mathrm{z} 140\left(\mathrm{M}^{+\bullet}, 30 \%\right), 122$ [(M-H $\left.\left.\mathrm{H}_{2} \mathrm{O}\right)^{+\bullet}, 60\right], 93$ (100), 79 (90), 77 (70), 70 (60), 55 (60); HRMS $\mathrm{M}^{+\bullet}$ calcd for $\mathrm{C}_{8} \mathrm{H}_{12} \mathrm{O}_{2}$ 140.0837, found 140.0842 .

Concentration of fraction B $\left(R_{\mathrm{f}}=0.4\right.$ in ethyl acetate) afforded compound $\mathbf{1 0}$ (48 mg, 48\%) as a white, crystalline solid that was identical, in all respects, with an authentic sample.

(1R,2S,3S,6R)-2-((tert-Butyldimethylsilyl)oxy)-4-methylbicyclo[4.1.0]hept-4-en-3ol (13). The magnetically stirred and deoxygenated solution of compound 7 (100 mg, $0.35 \mathrm{mmol})$ in dichloromethane $(100 \mathrm{~mL})$ maintained under a nitrogen atmosphere was subjected to irradiation with a Hanovia $450 \mathrm{~W}$ medium pressure mercury-vapor lamp for $5 \mathrm{~h}$. The cooled reaction mixture was concentrated under reduced pressure to give a brown oil that was subjected to flash column chromatography (silica, 1:9 $\rightarrow$ 1:4 v/v ethyl acetate/40-60 petroleum ether gradient elution) and thus affording two fractions, A and B.

Concentration of fraction A $\left(R_{\mathrm{f}}=0.7\right.$ in $3: 7 \mathrm{v} / \mathrm{v}$ ethyl acetate $/ 40-60$ petroleum ether) afforded compound 13 (30 mg, 33\%) as a colorless, crystalline solid, $\mathrm{mp}=32-$ $33{ }^{\circ} \mathrm{C}:[\alpha]_{\mathrm{D}}=+35.3\left(c\right.$ 1.0, $\left.\mathrm{CHCl}_{3}\right) .{ }^{1} \mathrm{H}$ NMR $\left(400 \mathrm{MHz}, \mathrm{CDCl}_{3}\right) \delta 5.71(\mathrm{~m}, 1 \mathrm{H}), 4.08$ (d, $J=5.9 \mathrm{~Hz}, 1 \mathrm{H}), 3.59$ (m, 1H), 1.97 (broad s, 1H), 1.74 (s, 3H), 1.33-1.17 (complex m, 2H), 0.99-0.94 (complex m, 1H), 0.92 (s, 9H), 0.32 (m, 1H), 0.14 (s, 3H), 0.12 (s, 3H); ${ }^{13} \mathrm{C}$ NMR (100 MHz, $\left.\mathrm{CDCl}_{3}\right) \delta 134.4,124.7,75.1,74.0,26.0,19.6$, 19.3, 18.7, 18.3, 12.0, -4.1, -4.6; IR $v_{\max } 3458,2929,2857,1254,1075,1003,835$, $775 \mathrm{~cm}^{-1}$; MS (EI, $\left.70 \mathrm{eV}\right) \mathrm{m} / \mathrm{z} 239\left[\left(\mathrm{M}-\mathrm{CH}_{3} \bullet\right)^{+}, 30 \%\right.$ ], $211(35), 197\left[\left(\mathrm{M}-\mathrm{C}_{4} \mathrm{H}_{9} \bullet\right)^{+}\right.$, 
35], 105 (60), 75 (100), 73 (95); HRMS (M- $\left.\mathrm{CH}_{3} \bullet\right)^{+}$calcd for $\mathrm{C}_{14} \mathrm{H}_{26} \mathrm{O}_{2} \mathrm{Si} 239.1467$, found 239.1468.

Concentration of fraction B $\left(R_{\mathrm{f}}=0.6\right.$ in $3: 7 \mathrm{v} / \mathrm{v}$ ethyl acetate $/ 40-60$ petroleum ether) afforded compound $\mathbf{1 1}$ (33 mg, 33\%) as a colorless, crystalline solid that was identical, in all respects, with an authentic sample.

(1S,2R)-3-Methylcyclohexa-3,5-diene-1,2-diyl (2E,2'E)-bis(But-2-enoate) (14). A magnetically stirred solution of (1S,2R)-3-methylcyclohexa-3,5-diene-1,2-diol (5) (2.40 g, $19.0 \mathrm{mmol})$ in dry THF $(284 \mathrm{~mL})$ was cooled to $-78{ }^{\circ} \mathrm{C}$ and then treated, dropwise over $0.5 \mathrm{~h}$, with $n$-BuLi $(12.5 \mathrm{~mL}$ of a $1.6 \mathrm{M}$ solution in hexane, 20.0 mmol). The resulting solution was stirred at $-78{ }^{\circ} \mathrm{C}$ for $0.17 \mathrm{~h}$ then treated with crotonoyl chloride $(1.80 \mathrm{~mL}, 19.02 \mathrm{mmol})$ over $0.33 \mathrm{~h}$. The resulting solution was stirred for $2 \mathrm{~h}$ at $-78^{\circ} \mathrm{C}$ then treated, dropwise over $0.5 \mathrm{~h}$, with $n$-BuLi (12.5 mL of a 1.6 $\mathrm{M}$ solution in hexane, $20.0 \mathrm{mmol}$ ). After $0.17 \mathrm{~h}$ another portion of crotonoyl chloride $(1.80 \mathrm{~mL}, 19.02 \mathrm{mmol})$ was added over $0.33 \mathrm{~h}$ then stirring of the recation mixture was continued at $-78{ }^{\circ} \mathrm{C}$ for $1 \mathrm{~h}$. The ensuing mixture was warmed to $22{ }^{\circ} \mathrm{C}$ then quenched with $\mathrm{NaHCO}_{3}$ (96 mL of a saturated aqueous solution) before being diluted with diethyl ether (390 mL). The separated organic phase was washed with $\mathrm{NH}_{4} \mathrm{Cl}(1 \times 96 \mathrm{~mL}$ of a saturated aqueous solution $)$ then dried $\left(\mathrm{MgSO}_{4}\right)$, filtered, and concentrated under reduced pressure. The residue thus obtained was subjected to flash column chromatography to give, after concentration of the appropriate fractions $\left(R_{\mathrm{f}}=\right.$ 0.5 in 1:4 v/v ethyl acetate/40-60 petroleum ether), compound 14 (4.04 g, 81\%) as a pale-yellow oil. The spectral data recorded on this material matched those reported previously. $^{8}$

(1S,2R)-3-Methylcyclohexa-3,5-diene-1,2-diyl $\quad\left(2 E, 2^{\prime} E\right)$-bis(4-Methylpent-2-eno ate) (15). A magnetically stirred solution of (1S,2R)-3-methylcyclohexa-3,5-diene- 
1,2-diol (5) $(2.40 \mathrm{~g}, 19.02 \mathrm{mmol})$ in dry THF $(284 \mathrm{~mL})$ was cooled to $-78{ }^{\circ} \mathrm{C}$ then treated, dropwise over $0.5 \mathrm{~h}$, with $n$-BuLi (12.5 mL of a $1.6 \mathrm{M}$ solution in hexane, $20.0 \mathrm{mmol}$ ). The resulting solution was stirred at $-78^{\circ} \mathrm{C}$ for $0.17 \mathrm{~h}$ then treated, dropwise over $0.33 \mathrm{~h}$, with (E)-4-methylpent-2-enoyl chloride ${ }^{14}(2.50 \mathrm{~mL}, 19.02$ mmol). The ensuing mixture was stirred at $-78^{\circ} \mathrm{C}$ for $2 \mathrm{~h}$ and again treated, dropwise over $0.5 \mathrm{~h}$, with $n$-BuLi (12.5 mL of a 1.6 M solution in hexane, $20.0 \mathrm{mmol}$ ). After 0.17 h further (E)-4-methylpent-2-enoyl chloride (1.80 mL, $19.02 \mathrm{mmol})$ was added, again dropwise over $0.33 \mathrm{~h}$, and stirring continued at $-78{ }^{\circ} \mathrm{C}$ for $1 \mathrm{~h}$. The reaction mixture was then allowed to warm to $22{ }^{\circ} \mathrm{C}$ before being quenched with $\mathrm{NaHCO}_{3}$ (96 $\mathrm{mL}$ of a saturated aqueous solution) and diluted with diethyl ether (390 mL). The separated organic phase was washed with $\mathrm{NH}_{4} \mathrm{Cl}(1 \times 96 \mathrm{~mL}$ of a saturated aqueous solution) then dried $\left(\mathrm{MgSO}_{4}\right)$, filtered and concentrated under reduced pressure. The residue thus obtained was subjected to flash column chromatography to give, after concentration of the appropriate fractions $\left(R_{\mathrm{f}}=0.6\right.$ in $1: 4 \mathrm{v} / \mathrm{v}$ ethyl acetate/40-60 petroleum ether), compound $15(4.47 \mathrm{~g}, 74 \%)$ as a pale-yellow oil, $[\alpha]_{\mathrm{D}}=+71.6(\mathrm{c}$ 1.0, $\left.\mathrm{CHCl}_{3}\right) .{ }^{1} \mathrm{H} \mathrm{NMR}\left(400 \mathrm{MHz}, \mathrm{CDCl}_{3}\right) \delta$ 6.98-6.88 (complex m, 2H), 6.06 (m, 1H), 5.88 (broadened d, $J=5.2 \mathrm{~Hz}, 1 \mathrm{H}$ ), 5.81-5.72 (complex m, 3H), 5.62 (s, 2H), 2.43 (m, 2H), 1.84 (s, 3H), 1.05-1.02 (complex m, 12H); ${ }^{13} \mathrm{C}$ NMR (100 MHz, $\left.\mathrm{CDCl}_{3}\right) \delta$ 166.5, 166.3, 156.5, 156.2, 134.7, 126.6, 123.0, 121.9, 118.3, 118.1, 69.5, 68.0, 31.1, 31.0, 21.3, 21.2, 19.8 (two signals obscured or overlapping); IR $v_{\max } 2963,1717,1651$, 1297, 1260, 1157, 982, $859 \mathrm{~cm}^{-1}$; MS (ESI, +ve) m/z 341 [(M+Na) $\left.{ }^{+}, 100 \%\right]$; HRMS $(\mathrm{M}+\mathrm{Na})^{+}$calcd for $\mathrm{C}_{19} \mathrm{H}_{26} \mathrm{O}_{4} 341.1729$, found 341.1728 .

3a,8-Dimethyl-2-oxo-2,3,3a,6,7,7a-hexahydro-3,6-methanobenzofuran-7-yl $\quad(E)$ But-2-enoate (16) and 6,8-Dimethyl-2-oxo-2,3,3a,6,7,7a-hexahydro-3,6methanobenzofuran-7-yl (E)-But-2-enoate (18). A magnetically stirred solution of 
compound 14 (4.04 g, $15.40 \mathrm{mmol})$ in toluene (97 mL) maintained under a nitrogen atmosphere was heated at reflux for $24 \mathrm{~h}$ then cooled to $22{ }^{\circ} \mathrm{C}$ and concentrated under reduced pressure. The resulting yellow oil was subjected to flash chromatography (silica, 1:19 v/v ethyl acetate/40-60 petroleum ether elution) to give two fractions, A and $\mathrm{B}$.

Concentration of fraction A $\left[R_{\mathrm{f}}=0.2(5)\right.$ in $1: 4 \mathrm{v} / \mathrm{v}$ ethyl acetate $/ 40-60$ petroleum ether] afforded compound 16 (1.62 g, 40\%) as a white, crystalline solid, $\mathrm{mp}=45-47^{\circ} \mathrm{C}$. The spectral data recorded on this material matched those reported previously. $^{8}$

Concentration of fraction B $\left(R_{\mathrm{f}}=0.2\right.$ in $1: 4 \mathrm{v} / \mathrm{v}$ ethyl acetate/40-60 petroleum ether) afforded compound 18 (1.06 g, 26\%) as a clear, colorless oil. The spectral data recorded on this material matched those reported previously. ${ }^{8}$

8-Isopropyl-3a-methyl-2-oxo-2,3,3a,6,7,7a-hexahydro-3,6-methanobenzofuran-7yl (E)-4-Methylpent-2-enoate (17) and 8-Isopropyl-6-methyl-2-oxo-2,3,3a,6,7,7ahexahydro-3,6-methanobenzofuran-7-yl $\quad(E)-4-M e t h y l p e n t-2-e n o a t e \quad(19)$. A magnetically stirred solution of compound 15 (4.47 g, $14.04 \mathrm{mmol})$ in toluene (88 $\mathrm{mL}$ ) maintained under a nitrogen atmosphere was heated at reflux for $72 \mathrm{~h}$ then cooled to $22{ }^{\circ} \mathrm{C}$ and concentrated under reduced pressure. The resulting yellow oil was subjected to flash chromatography (silica, 1:19 v/v ethyl acetate/40-60 petroleum ether elution) and so affording two fractions, A and B.

Concentration of fraction A $\left(R_{\mathrm{f}}=0.4\right.$ in $1: 4 \mathrm{v} / \mathrm{v}$ ethyl acetate/40-60 petroleum ether) afforded compound 17 (1.83 g, 41\%) as a white, crystalline solid, $\mathrm{mp}=69-71$ ${ }^{\circ} \mathrm{C},[\alpha]_{\mathrm{D}}=-115.4\left(\right.$ c 1.0, $\left.\mathrm{CHCl}_{3}\right) .{ }^{1} \mathrm{H}$ NMR $\left(400 \mathrm{MHz}, \mathrm{CDCl}_{3}\right) \delta 6.97(\mathrm{dd}, J=15.7$ and $6.5 \mathrm{~Hz}, 1 \mathrm{H}), 6.38(\mathrm{t}, J=7.6 \mathrm{~Hz}, 1 \mathrm{H}), 5.84(\mathrm{~m}, 2 \mathrm{H}), 4.37$ (dd, $J=6.7$ and $2.2 \mathrm{~Hz}$, 1H), 4.24 (d, $J=6.7 \mathrm{~Hz}, 1 \mathrm{H}), 2.98(\mathrm{~m}, 1 \mathrm{H}), 2.47$ (m, 1H), 1.93-1.88 (complex m, 
2H), 1.40 (s, 3H), 1.27 (m, 1H), 1.08 (s, 3H), 1.06 (s, 3H), 0.97 (d, J = 6.5 Hz, 3H), $0.90(\mathrm{~d}, J=6.6 \mathrm{~Hz}, 3 \mathrm{H}) ;{ }^{13} \mathrm{C} \mathrm{NMR}\left(100 \mathrm{MHz}, \mathrm{CDCl}_{3}\right) \delta 179.3,166.5,156.8,133.1$, 132.2, 117.9, 77.3, 70.6, 49.7, 46.9, 45.1, 38.8, 31.1, 30.5, 21.3, 21.2, 20.9, 20.8, 20.7; IR $v_{\max }$ 2962, 1783, 1717, 1264, 1160, 1038, 1009, $902 \mathrm{~cm}^{-1}$; MS (EI, $\left.70 \mathrm{eV}\right) \mathrm{m} / \mathrm{z} 318$ (M+•, 5\%), 156 (15), 133 (10), 97 (100), 69 (10), 41 (20); HRMS $\mathrm{M}^{+\bullet}$ calcd for $\mathrm{C}_{19} \mathrm{H}_{26} \mathrm{O}_{4} 318.1831$, found 318.1834.

Concentration of fraction $\mathrm{B}\left[R_{\mathrm{f}}=0.3(5)\right.$ in $1: 4 \mathrm{v} / \mathrm{v}$ ethyl acetate $/ 40-60$ petroleum ether] afforded compound 19 (537 mg, 12\%) as a clear, colorless oil, $[\alpha]_{\mathrm{D}}$ $=+176.0\left(\right.$ c 1.0, $\left.\mathrm{CHCl}_{3}\right) .{ }^{1} \mathrm{H} \mathrm{NMR}\left(400 \mathrm{MHz}, \mathrm{CDCl}_{3}\right) \delta 6.97(\mathrm{dd}, J=15.7$ and $6.5 \mathrm{~Hz}$, 1H), 6.09 (d, $J=8.2 \mathrm{~Hz}, 1 \mathrm{H}$ ), 6.00 (dd, $J=8.2$ and $6.6 \mathrm{~Hz}, 1 \mathrm{H}$ ), 5.83 (dd, $J=15.7$ and $1.5 \mathrm{~Hz}, 1 \mathrm{H}), 4.57$ (m, 1H), 4.05 (d, $J=6.7 \mathrm{~Hz}, 1 \mathrm{H}), 3.45$ (m, 1H), 2.47 (m, 1H), 2.30 (m, 1H), 2.16 (m, 1H), 2.01 (m, 1H), 1.24 (s, 3H), 1.08 (s, 3H), 1.06 (s, 3H), 0.99 (d, $J=6.9 \mathrm{~Hz}, 3 \mathrm{H}), 0.61(\mathrm{~d}, J=6.8 \mathrm{~Hz}, 3 \mathrm{H}) ;{ }^{13} \mathrm{C} \mathrm{NMR}\left(100 \mathrm{MHz}, \mathrm{CDCl}_{3}\right) \delta 179.7$, 166.7, 156.8, 140.3, 124.6, 117.8, 74.4, 72.9, 48.0, 42.2, 40.6, 40.2, 31.1, 27.4, 22.8, 21.3, 21.2, 19.9, 19.1; IR $v_{\max }$ 2961, 1786, 1718, 1264, 1158, 1027, 982, $907 \mathrm{~cm}^{-1}$;

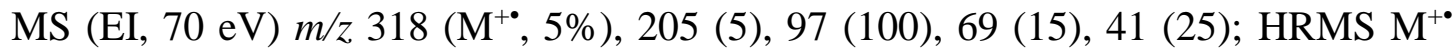
calcd for $\mathrm{C}_{19} \mathrm{H}_{26} \mathrm{O}_{4} 318.1831$, found 318.1832.

\section{7-Hydroxy-6,8-dimethyl-3a,6,7,7a-tetrahydro-3,6-methanobenzofuran-2(3H)-one}

(20). A magnetically stirred solution of compound 18 (918 $\mathrm{mg}, 3.50 \mathrm{mmol}$ ) in metahnol $(55 \mathrm{~mL})$ maintained at $0{ }^{\circ} \mathrm{C}$ was treated, in one portion, with potassium carbonate (242 mg, $1.75 \mathrm{mmol}$ ). The ensuing mixture was stirred at $0{ }^{\circ} \mathrm{C}$ for a further $0.5 \mathrm{~h}$ then warmed to $22^{\circ} \mathrm{C}$, stirred at this temperature for $1 \mathrm{~h}$ then re-cooled to $0{ }^{\circ} \mathrm{C}$ and treated with water $(30 \mathrm{~mL})$ before being concentrated under reduced pressure. The residue thus obtained was extracted with ethyl acetate $(3 \times 100 \mathrm{~mL})$ and the combined organic phases then dried $\left(\mathrm{MgSO}_{4}\right)$, filtered, and concentrated under 
reduced pressure. The resulting light-yellow oil was subjected to flash column chromatography (silica, 3:7 $\rightarrow$ 1:1 v/v ethyl acetate/40-60 petroleum ether gradient elution) to give, after concentration of the appropriate fractions $\left(R_{\mathrm{f}}=0.1\right.$ in $3: 7 \mathrm{v} / \mathrm{v}$ ethyl acetate/40-60 petroleum ether), compound 20 (503 mg, 74\%) as a white, crystalline solid, $\mathrm{mp}=114-116{ }^{\circ} \mathrm{C},[\alpha]_{\mathrm{D}}=+67.5\left(c\right.$ 1.0, $\left.\mathrm{CHCl}_{3}\right) .{ }^{1} \mathrm{H}$ NMR $(400 \mathrm{MHz}$, $\left.\mathrm{CDCl}_{3}\right) \delta$ 6.06-6.00 (complex m, 2H), 4.39 (t, $\left.J=6.0 \mathrm{~Hz}, 1 \mathrm{H}\right), 3.43(\mathrm{~m}, 1 \mathrm{H}), 3.26$ (t, $J$ = $6.7 \mathrm{~Hz}, 1 \mathrm{H}), 2.23(\mathrm{~d}, J=6.8 \mathrm{~Hz}, 1 \mathrm{H}), 2.11(\mathrm{~m}, 1 \mathrm{H}), 2.01$ (d, $J=4.5 \mathrm{~Hz}, 1 \mathrm{H}), 1.25$ (s, 3H), 0.89 (d, $J=7.1 \mathrm{~Hz}, 3 \mathrm{H}) ;{ }^{13} \mathrm{C}$ NMR (100 MHz, $\left.\mathrm{CDCl}_{3}\right) \delta 178.9,141.2,124.2$, 74.9, 71.9, 46.9, 44.1, 40.4, 36.5, 18.8, 17.3; IR $v_{\max } 3417,2968,1760,1750,1184$, 1102, 1015, $713 \mathrm{~cm}^{-1}$; MS (ESI, +ve) m/z $217\left[(\mathrm{M}+\mathrm{Na})^{+}, 100 \%\right]$; HRS $(\mathrm{M}+\mathrm{Na})^{+}$ calcd for $\mathrm{C}_{11} \mathrm{H}_{14} \mathrm{O}_{3} 217.0841$, found 217.0844.

\section{7-Hydroxy-8-isopropyl-6-methyl-3a,6,7,7a-tetrahydro-3,6-methanobenzofuran-}

2(3H)-one (21). A magnetically stirred solution of compound 19 (1.11 g, $3.50 \mathrm{mmol})$ in methanol $(55 \mathrm{~mL})$ maintained at $0{ }^{\circ} \mathrm{C}$ was treated, in one portion, with potassium carbonate (242 mg, $1.75 \mathrm{mmol}$ ). After being kept for $0.5 \mathrm{~h}$ at $0{ }^{\circ} \mathrm{C}$ the reaction mixture was warmed to $22{ }^{\circ} \mathrm{C}$, maintained at this temperature for $1 \mathrm{~h}$ then re-cooled to $0{ }^{\circ} \mathrm{C}$ and treated with water $(30 \mathrm{~mL})$ before being concentrated under reduced pressure. The residue thus obtained was extracted with ethyl acetate $(3 \times 100 \mathrm{~mL})$ and the combined organic phases were then dried $\left(\mathrm{MgSO}_{4}\right)$, filtered, and concentrated under reduced pressure to give a light-yellow oil. This material was subjected to flash column chromatography (silica, 3:17 $\rightarrow$ 3:7 v/v ethyl acetate/40-60 petroleum ether gradient elution) and gave, after concentration of the appropriate fractions $\left(R_{\mathrm{f}}=0.3\right.$ in 3:7 v/v ethyl acetate/40-60 petroleum ether), compound 21 (590 mg, 76\%) as a white solid, mp $=120-122{ }^{\circ} \mathrm{C},[\alpha]_{\mathrm{D}}=+100.7\left(c\right.$ 1.0, $\left.\mathrm{CHCl}_{3}\right) .{ }^{1} \mathrm{H} \mathrm{NMR}\left(400 \mathrm{MHz}, \mathrm{CDCl}_{3}\right) \delta$ 6.09 (d, $J=8.1 \mathrm{~Hz}, 1 \mathrm{H}), 5.93(\mathrm{~m}, 1 \mathrm{H}), 4.35$ (m, 1H), $3.43(\mathrm{~m}, 1 \mathrm{H}), 3.12(\mathrm{~d}, J=6.9$ 
Hz, 1H), 2.28 (m, 1H), 2.03-1.95 (complex m, 2H), 1.26 (s, 3H), 0.97 (d, $J=6.7 \mathrm{~Hz}$, 3H), 0.60 (d, $J=6.7 \mathrm{~Hz}, 3 \mathrm{H}$ ) (signal due to O-H group proton not observed); ${ }^{13} \mathrm{C}$ NMR (100 MHz, $\left.\mathrm{CDCl}_{3}\right) \delta 179.3,141.8,123.3,74.8,72.9,47.6,44.0,40.2(3)$, 40.1(7), 27.3, 22.7, 19.8, 18.8; IR $v_{\max } 3425,2958,1765,1170,1100,978,723 \mathrm{~cm}^{-1}$; MS (ESI, +ve) m/z $467\left[(2 \mathrm{M}+\mathrm{Na})^{+}, 30 \%\right], 245\left[(\mathrm{M}+\mathrm{Na})^{+}, 100\right], 223\left[(\mathrm{M}+\mathrm{H})^{+}, 70\right]$; HRMS (M+Na) ${ }^{+}$calcd for $\mathrm{C}_{13} \mathrm{H}_{18} \mathrm{O}_{3} 245.1154$, found 245.1156 .

\section{6,8-Dimethyl-3a,7a-dihydro-3,6-methanobenzofuran-2,7(3H,6H)-dione (22). A} magnetically stirred solution of compound 20 (466 mg, $2.40 \mathrm{mmol}$ ) in dry dichloromethane $(78 \mathrm{~mL})$ maintained at $0{ }^{\circ} \mathrm{C}$ under an atmosphere of nitrogen was treated with pyridine $(1.2 \mathrm{~mL}, 15.05 \mathrm{mmol})$ then the Dess-Martin periodinane (1.83 g, $4.32 \mathrm{mmol}$ ). After a further $0.5 \mathrm{~h}$ the reaction mixture was warmed to $22{ }^{\circ} \mathrm{C}$, maintained at this temperature for $3 \mathrm{~h}$ then poured into water $(100 \mathrm{~mL})$ and extracted with dichloromethane $(3 \times 100 \mathrm{~mL})$. The combined organic phases were washed with $\mathrm{NaOH}(1 \times 100 \mathrm{ml}$ of a $1 \mathrm{M}$ aqueous solution $)$ then $\mathrm{HCl}(1 \times 100 \mathrm{ml}$ of a $1 \mathrm{M}$ aqueous solution) before being dried $\left(\mathrm{MgSO}_{4}\right)$, filtered, and concentrated under reduced pressure. The residue thus obtained was subjected to flash column chromatography (silica, 1:9 $\rightarrow$ 3:17 v/v ethyl acetate/40-60 petroleum ether gradient elution) to give, after concentration of the appropriate fractions $\left(R_{\mathrm{f}}=0.3\right.$ in $3: 7 \mathrm{v} / \mathrm{v}$ ethyl acetate/40-60 petroleum ether), compound 22 (397 $\mathrm{mg}, 86 \%$ ) as a white, crystalline solid, $\mathrm{mp}=121-124{ }^{\circ} \mathrm{C},[\alpha]_{\mathrm{D}}=+518.8\left(\right.$ c 1.0, $\left.\mathrm{CHCl}_{3}\right) .{ }^{1} \mathrm{H}$ NMR (400 $\left.\mathrm{MHz}, \mathrm{CDCl}_{3}\right) \delta 6.32(\mathrm{dd}, J=8.1$ and $6.5 \mathrm{~Hz}, 1 \mathrm{H}), 6.02(\mathrm{~d}, J=8.1 \mathrm{~Hz}, 1 \mathrm{H}), 4.15$ (dd, $J=5.0$ and $1.0 \mathrm{~Hz}, 1 \mathrm{H}), 3.66(\mathrm{~m}, 1 \mathrm{H}), 2.37(\mathrm{~d}, J=5.0 \mathrm{~Hz}, 1 \mathrm{H}), 2.21(\mathrm{~m}, 1 \mathrm{H}), 1.32(\mathrm{~s}$, 3H), 1.02 (d, $J=7.0 \mathrm{~Hz}, 3 \mathrm{H}) ;{ }^{13} \mathrm{C}$ NMR $\left(100 \mathrm{MHz}, \mathrm{CDCl}_{3}\right) \delta$ 201.5, 177.8, 136.7, 128.3, 73.9, 53.8, 47.1, 41.3, 40.9, 16.1, 15.2; IR $v_{\max } 2976,2937,1785,1736,1159$, 989, 837, $713 \mathrm{~cm}^{-1}$; MS (ESI, +ve) m/z $247\left[(\mathrm{M}+\mathrm{MeOH}+\mathrm{Na})^{+}, 100 \%\right], 215\left[(\mathrm{M}+\mathrm{Na})^{+}\right.$, 
20]; HRMS (M+MeOH+Na) ${ }^{+}$calcd for $\mathrm{C}_{12} \mathrm{H}_{16} \mathrm{O}_{4}$ 247.0946, found 247.0946.

\section{8-Isopropyl-6-methyl-3a,7a-dihydro-3,6-methanobenzofuran-2,7(3H,6H)-dione}

(23). A magnetically stirred solution of compound 21 (533 mg, $2.40 \mathrm{mmol}$ ) in dry dichloromethane $(78 \mathrm{~mL})$ maintained at $0{ }^{\circ} \mathrm{C}$ under an atmosphere of nitrogen was treated with pyridine $(1.2 \mathrm{~mL}, 15.05 \mathrm{mmol})$ then the Dess-Martin periodinane (1.83 $\mathrm{g}$, $4.32 \mathrm{mmol}$ ). After a further $0.5 \mathrm{~h}$ the reaction mixture was warmed to $22{ }^{\circ} \mathrm{C}$, stirred at this temperature for $3 \mathrm{~h}$ then poured into water $(100 \mathrm{~mL})$ and extracted with dichloromethane $(3 \times 100 \mathrm{~mL})$. The combined organic phases were washed with $\mathrm{NaOH}(1 \times 100 \mathrm{ml}$ of a $1 \mathrm{M}$ aqueous solution $)$ then $\mathrm{HCl}(1 \times 100 \mathrm{ml}$ of a $1 \mathrm{M}$ aqueous solution) before being dried $\left(\mathrm{MgSO}_{4}\right)$, filtered, and concentrated under reduced pressure. The residue thus obtained was subjected to flash column chromatography (silica, 3:17 $\rightarrow$ 3:7 v/v ethyl acetate/40-60 petroleum ether gradient elution) and gave, after concentration of the appropriate fractions $\left(R_{\mathrm{f}}=0.3\right.$ in $3: 7 \mathrm{v} / \mathrm{v}$ ethyl acetate/40-60 petroleum ether), compound 23 (433 $\mathrm{mg}, 82 \%$ ) as a white, crystalline solid, $\mathrm{mp}=114-116{ }^{\circ} \mathrm{C},[\alpha]_{\mathrm{D}}=+396.1\left(\right.$ c $\left.0.8, \mathrm{CHCl}_{3}\right) .{ }^{1} \mathrm{H}$ NMR $(400$ $\left.\mathrm{MHz}, \mathrm{CDCl}_{3}\right) \delta 6.19(\mathrm{dd}, J=8.1$ and $6.6 \mathrm{~Hz}, 1 \mathrm{H}), 6.04(\mathrm{~m}, 1 \mathrm{H}), 4.12$ (dd, $J=5.0$ and $1.1 \mathrm{~Hz}, 1 \mathrm{H}), 3.67$ (m, 1H), 2.64 (m, 1H), 2.11 (m, 1H), 1.99 (m, 1H), 1.36 (s, 3H), $1.01(\mathrm{~d}, J=6.9 \mathrm{~Hz}, 3 \mathrm{H}), 0.72(\mathrm{~d}, J=6.8 \mathrm{~Hz}, 3 \mathrm{H}) ;{ }^{13} \mathrm{C} \mathrm{NMR}\left(100 \mathrm{MHz}, \mathrm{CDCl}_{3}\right) \delta$ 200.7, 178.5, 136.8, 127.3, 73.9, 53.6, 51.7, 40.9, 40.4, 28.2, 22.8, 20.0, 15.3; IR $v_{\max }$ 2961, 1802, 1786, 1734, 1151, 1002, 981, $899 \mathrm{~cm}^{-1}$; MS (ESI, +ve) m/z 275 $\left[(\mathrm{M}+\mathrm{MeOH}+\mathrm{Na})^{+}, 100 \%\right], 243\left[(\mathrm{M}+\mathrm{Na})^{+}, 40\right] ; \mathrm{HRMS}(\mathrm{M}+\mathrm{MeOH}+\mathrm{Na})^{+}$calcd for $\mathrm{C}_{13} \mathrm{H}_{16} \mathrm{O}_{3} 275.1259$, found 275.1251.

\section{7-Hydroxy-3a,8-dimethyl-3a,6,7,7a-tetrahydro-3,6-methanobenzofuran-2(3H)-}

one (24). A magnetically stirred solution of compound 16 (918 mg, $3.50 \mathrm{mmol}$ ) in methanol (55 mL) maintained at $0{ }^{\circ} \mathrm{C}$ under an atmosphere of nitrogen was treated, in 
one portion, with potassium carbonate $(242 \mathrm{mg}, 1.75 \mathrm{mmol})$. After a further $0.5 \mathrm{~h}$ the reaction mixture was warmed to $22{ }^{\circ} \mathrm{C}$, stirred at this temperature for $1 \mathrm{~h}$ then recooled to $0{ }^{\circ} \mathrm{C}$ and treated with water $(30 \mathrm{~mL}$ ) before being concentrated under reduced pressure. The residue thus obtained was extracted with ethyl acetate $(3 \times 100$ $\mathrm{mL})$ and the combined organic phases then dried $\left(\mathrm{MgSO}_{4}\right)$, filtered, and concentrated under reduced pressure to give a light-yellow oil. This material was subjected to flash column chromatography (silica, 1:4 $\rightarrow$ 3:7 v/v ethyl acetate/40-60 petroleum ether gradient elution) and gave, after concentration of the appropriate fractions $\left(R_{\mathrm{f}}=0.2\right.$ in 3:7 v/v ethyl acetate/40-60 petroleum ether), compound 24 (538 mg, 79\%) as a white, crystalline solid, $\mathrm{mp}=146-148{ }^{\circ} \mathrm{C},[\alpha]_{\mathrm{D}}=+15.8\left(c\right.$ 1.0, $\left.\mathrm{CHCl}_{3}\right) .{ }^{1} \mathrm{H}$ NMR $(400 \mathrm{MHz}$, $\left.\mathrm{CDCl}_{3}\right) \delta 6.36(\mathrm{t}, J=7.6 \mathrm{~Hz}, 1 \mathrm{H}), 5.81(\mathrm{dd}, J=7.6 \mathrm{~Hz}$ and $1.0 \mathrm{~Hz}, 1 \mathrm{H}), 3.98(\mathrm{~d}, J=$ $6.9 \mathrm{~Hz}, 1 \mathrm{H}$ ), 3.57 (dd, $J=6.9$ and $2.2 \mathrm{~Hz}, 1 \mathrm{H}), 2.68$ (m, 1H), 2.47 (m, 2H), 1.68 (s, 1H), 1.39 (s, 3H), 0.93 (d, $\left.J=7.1 \mathrm{~Hz}, 3 \mathrm{H}) ;{ }^{13} \mathrm{C} \mathrm{NMR} \mathrm{(100} \mathrm{MHz,} \mathrm{CDCl}_{3}\right) \delta$ 179.0, 135.4, 130.8, 79.0, 69.0, 52.2, 45.2, 45.0, 33.1, 21.1, 20.1; IR $v_{\max } 3402,2964,1740$, 1347, 1182, 1085, 992, 948, 713, $703 \mathrm{~cm}^{-1}$; MS (ESI, +ve) m/z 217 [(M+Na) $\left.{ }^{+}, 100 \%\right]$; HRMS (M+Na) ${ }^{+}$calcd for $\mathrm{C}_{11} \mathrm{H}_{14} \mathrm{O}_{3} 217.0841$, found 217.0842 .

\section{7-Hydroxy-8-isopropyl-3a-methyl-3a,6,7,7a-tetrahydro-3,6-methanobenzofuran-}

2(3H)-one (25). A magnetically stirred solution of compound 17 (1.11 g, $3.50 \mathrm{mmol}$ ) in methanol $(55 \mathrm{~mL})$ maintained at $0{ }^{\circ} \mathrm{C}$ under an atmosphere of nitrogen was treated, in one portion, with potassium carbonate (242 mg, $1.75 \mathrm{mmol}$ ). After a further $0.5 \mathrm{~h}$ at $0{ }^{\circ} \mathrm{C}$, the reaction mixture was warmed to $22{ }^{\circ} \mathrm{C}$, stirred at this temperature for $1 \mathrm{~h}$ then re-cooled to $0{ }^{\circ} \mathrm{C}$ and treated with water $(30 \mathrm{~mL})$ before being concentrated under reduced pressure. The ensuing mixture was extracted with ethyl acetate $(3 \times$ $100 \mathrm{~mL})$ and the combined organic phases were dried $\left(\mathrm{MgSO}_{4}\right)$, filtered, and concentrated under reduced pressure. The residue thus obtained was subjected to flash 
column chromatography (silica, 3:7 $\rightarrow$ 1:1 v/v ethyl acetate/40-60 petroleum ether gradient elution) and gave, after concentration of the appropriate fractions $\left(R_{\mathrm{f}}=0.2\right.$ in 3:7 v/v ethyl acetate/40-60 petroleum ether), compound 25 (732 mg, 94\%) as a white, crystalline solid, $\mathrm{mp}=60-61{ }^{\circ} \mathrm{C},[\alpha]_{\mathrm{D}}=+2.8\left(c \mathrm{c} 0.9, \mathrm{CHCl}_{3}\right) .{ }^{1} \mathrm{H}$ NMR $(400 \mathrm{MHz}$, $\left.\mathrm{CDCl}_{3}\right) \delta 6.35(\mathrm{t}, J=8.3 \mathrm{~Hz}, 1 \mathrm{H}), 5.78$ (dd, $J=8.3$ and $\left.1.2 \mathrm{~Hz}, 1 \mathrm{H}\right), 3.99$ (d, $J=6.9$ Hz, 1H), 3.54 (dd, $J=6.9$ and $2.3 \mathrm{~Hz}, 1 \mathrm{H}), 2.89$ (m, 1H), 2.48 (broad s, 1H), 1.85 (m, 2H), 1.38 (s, 3H), 1.25 (m, 1H), 0.93 (d, $J=6.5 \mathrm{~Hz}, 3 \mathrm{H}), 0.87$ (d, $J=6.6 \mathrm{~Hz}, 3 \mathrm{H}) ;{ }^{13} \mathrm{C}$ NMR (100 MHz, $\left.\mathrm{CDCl}_{3}\right) \delta 179.1,134.6,131.1,79.3,68.9,49.7,46.4,44.9,41.7$, 30.7, 21.1, 20.8(0), 20.7(6); IR $v_{\max } 3452,2962,1778,1166,1117,993,706 \mathrm{~cm}^{-1}$; MS (ESI, +ve) m/z 245 [(M+Na) , 40\%], 223 [(M+H)+, 100], 205 (50), 159 (100), 109 (90), 90 (70); HRMS (M+H) ${ }^{+}$calcd for $\mathrm{C}_{13} \mathrm{H}_{18} \mathrm{O}_{3} 223.1334$ found, 223.1331.

3a,8-Dimethyl-3a,7a-dihydro-3,6-methanobenzofuran-2,7(3H,6H)-dione (26). A magnetically stirred solution of compound 24 (466 $\mathrm{mg}, 2.40 \mathrm{mmol}$ ) in dry dichloromethane (78 mL) maintained at $0{ }^{\circ} \mathrm{C}$ under an atmosphere of nitrogen was treated with pyridine $(1.2 \mathrm{~mL}, 15.05 \mathrm{mmol})$ then the Dess-Martin periodinane (1.83 $\mathrm{g}$, $4.32 \mathrm{mmol}$ ). After being kept at $0{ }^{\circ} \mathrm{C}$ for a further $0.5 \mathrm{~h}$ the reaction mixture was warmed to $22{ }^{\circ} \mathrm{C}$ and maintained at this temperature for $3 \mathrm{~h}$ before being poured into water $(100 \mathrm{~mL})$ and extracted with dichloromethane $(3 \times 100 \mathrm{~mL})$. The combined organic phases were washed with $\mathrm{NaOH}(1 \times 100 \mathrm{ml}$ of a $1 \mathrm{M}$ aqueous solution $)$ and $\mathrm{HCl}(1 \times 100 \mathrm{ml}$ of a $1 \mathrm{M}$ aqueous solution $)$ then dried $\left(\mathrm{MgSO}_{4}\right)$, filtered, and concentrated under reduced pressure. The residue thus obtained was subjected to flash column chromatography (silica, $1: 9 \rightarrow 3: 17 \mathrm{v} / \mathrm{v}$ ethyl acetate/40-60 petroleum ether gradient elution) and gave, after concentration of the appropriate fractions $\left(R_{\mathrm{f}}=0.3\right.$ in 3:7 v/v ethyl acetate/40-60 petroleum ether), compound 26 (434 mg, 94\%) as a white, crystalline solid, $\mathrm{mp}=135-136{ }^{\circ} \mathrm{C},[\alpha]_{\mathrm{D}}=-445.7$ (c 1.0, $\left.\mathrm{CHCl}_{3}\right) .{ }^{1} \mathrm{H}$ NMR $(400$ 
$\left.\mathrm{MHz}, \mathrm{CDCl}_{3}\right) \delta 6.37(\mathrm{t}, J=7.5 \mathrm{~Hz}, 1 \mathrm{H}), 6.13(\mathrm{dd}, J=7.5$ and $1.0 \mathrm{~Hz}, 1 \mathrm{H}$ ), 3.75 (s, 1H), 3.27 (dd, $J=6.7$ and $3.1 \mathrm{~Hz}, 1 \mathrm{H}), 2.59$ (m, 1H), 1.98 (s, 1H), 1.47 (s, 3H), 1.02 (d, $J=7.0 \mathrm{~Hz}, 3 \mathrm{H}) ;{ }^{13} \mathrm{C}$ NMR $\left(100 \mathrm{MHz}, \mathrm{CDCl}_{3}\right) \delta 200.0,177.7,134.5,130.4,78.5$, 54.0, 51.9, 46.5, 37.2, 19.9, 18.4; IR $v_{\max } 2964,1788,1742,1312,1161,995,875$, $723 \mathrm{~cm}^{-1} ; \quad$ MS $\quad(\mathrm{ESI}, \quad+\mathrm{ve}) \quad \mathrm{m} / \mathrm{z} \quad 247 \quad\left[(\mathrm{M}+\mathrm{MeOH}+\mathrm{Na})^{+}, \quad 100 \%\right] ; \quad \mathrm{HRMS}$ $(\mathrm{M}+\mathrm{MeOH}+\mathrm{Na})^{+}$calcd for $\mathrm{C}_{11} \mathrm{H}_{12} \mathrm{O}_{3} 247.0946$, found 247.0943 .

\section{8-Isopropyl-3a-methyl-3a,7a-dihydro-3,6-methanobenzofuran-2,7(3H,6H)-dione}

(27). A magnetically stirred solution of compound 25 (533 mg, $2.40 \mathrm{mmol}$ ) in dry dichloromethane $(78 \mathrm{~mL})$ maintained at $0{ }^{\circ} \mathrm{C}$ under an atmosphere of nitrogen was treated with pyridine $(1.2 \mathrm{~mL}, 15.05 \mathrm{mmol})$ then the Dess-Martin periodinane (1.83 g, $4.32 \mathrm{mmol}$ ). After a further $0.5 \mathrm{~h}$ at $0{ }^{\circ} \mathrm{C}$ the reaction mixture was warmed to $22{ }^{\circ} \mathrm{C}$ and then stirred at this temperature for $3 \mathrm{~h}$ before being poured into water (100 mL) and extracted with dichloromethane $(3 \times 100 \mathrm{~mL})$. The combined organic phases were washed with $\mathrm{NaOH}(1 \times 100 \mathrm{ml}$ of a $1 \mathrm{M}$ aqueous solution $)$ and $\mathrm{HCl}(1 \times 100 \mathrm{ml}$ of a $1 \mathrm{M}$ aqueous solution) then dried $\left(\mathrm{MgSO}_{4}\right)$, filtered, and concentrated under reduced pressure. The residue thus obtained was subjected to flash column chromatography (silica, 1:9 $\rightarrow$ 3:17 v/v ethyl acetate/40-60 petroleum ether gradient elution) and gave, after concentration of the appropriate fractions $\left(R_{\mathrm{f}}=0.5\right.$ in 3:7 v/v ethyl acetate/40-60 petroleum ether), compound 27 (413 mg, 78\%) as a white, crystalline solid, $\mathrm{mp}=$ $121-123{ }^{\circ} \mathrm{C},[\alpha]_{\mathrm{D}}=-332.5\left(c 1.0, \mathrm{CHCl}_{3}\right) .{ }^{1} \mathrm{H} \mathrm{NMR}\left(400 \mathrm{MHz}, \mathrm{CDCl}_{3}\right) \delta 6.37(\mathrm{t}, J=$ $8.2 \mathrm{~Hz}, 1 \mathrm{H}$ ), 6.09 (dd, $J=8.2$ and $1.1 \mathrm{~Hz}, 1 \mathrm{H}$ ), 3.77 (s, 1H), 3.49 (dd, $J=6.7$ and 3.1 Hz, 1H), 2.19 (s, 1H), 1.93 (dd, $J=10.2$ and $2.5 \mathrm{~Hz}, 1 \mathrm{H}), 1.47$ (s, 3H), 1.42 (m, 1H), $1.00(\mathrm{~d}, J=6.5 \mathrm{~Hz}, 3 \mathrm{H}), 0.89$ (d, $J=6.6 \mathrm{~Hz}, 3 \mathrm{H}) ;{ }^{13} \mathrm{C}$ NMR $\left(100 \mathrm{MHz}, \mathrm{CDCl}_{3}\right) \delta$ 200.4, 177.7, 134.8, 129.8, 78.8, 51.1, 50.2, 49.4, 46.2, 29.2, 20.6, 20.4, 19.9; IR $v_{\max }$ 2968, 17909, 1775, 1741, 1160, 985, $713 \mathrm{~cm}^{-1}$; MS (ESI, +ve) $\mathrm{m} / \mathrm{z} 275$ 
$\left[(\mathrm{M}+\mathrm{MeOH}+\mathrm{Na})^{+}, 100 \%\right], 221\left[(\mathrm{M}+\mathrm{H})^{+}, 10\right]$; HRMS $(\mathrm{M}+\mathrm{MeOH}+\mathrm{Na})^{+}$calcd for $\mathrm{C}_{13} \mathrm{H}_{16} \mathrm{O}_{3} 275.1259$, found 275.1260.

$\left(1 \mathrm{a} S, 1 \mathrm{a}^{\prime} R, 3 \mathrm{a} S, 4 S, 4 \mathrm{a} R, 4 \mathrm{a}^{\prime} R\right)-4,4 \mathrm{a}-D$ imethylhexahydro-1H-cyclopropa[3,4]pent-

aleno[1,6-bc]furan-1,3(1aH)-dione (28). A deoxygenated and magnetically stirred solution of compound $22(108 \mathrm{mg}, 0.56 \mathrm{mmol})$ and acetophenone $(90 \mu \mathrm{L}, 0.77 \mathrm{mmol})$ in acetone $(100 \mathrm{~mL})$ maintained under a nitrogen atmosphere was subjected to irradiation with a Hanovia 450W medium pressure mercury-vapor lamp for $0.83 \mathrm{~h}$. The reaction mixture was then cooled and concentrated under reduced pressure to give a brown oil that was subjected to flash column chromatography (silica, 3:17 $\rightarrow$ 3:7 v/v ethyl acetate/40-60 petroleum ether gradient elution). Concentration of the appropriate fractions $\left(R_{\mathrm{f}}=0.3\right.$ in $1: 1 \mathrm{v} / \mathrm{v}$ ethyl acetate/40-60 petroleum ether) then gave compound 28 (44 mg, 40\%) as a pale-yellow oil, $[\alpha]_{\mathrm{D}}=+5.1\left(\mathrm{c} \mathrm{1.0}, \mathrm{CHCl}_{3}\right) .{ }^{1} \mathrm{H}$ NMR (400 MHz, $\left.\mathrm{CDCl}_{3}\right) \delta 4.57$ (dd, $J=9.0$ and $\left.2.2 \mathrm{~Hz}, 1 \mathrm{H}\right), 3.59$ (m, 1H), 2.80 (t, $J$ $=8.0 \mathrm{~Hz}, 1 \mathrm{H}), 2.60(\mathrm{~m}, 1 \mathrm{H}), 2.45(\mathrm{~m}, 1 \mathrm{H}), 1.98(\mathrm{~m}, 1 \mathrm{H}), 1.30(\mathrm{~d}, J=7.2 \mathrm{~Hz}, 3 \mathrm{H})$, 1.22 (s, 3H); ${ }^{13} \mathrm{C}$ NMR (100 MHz, $\left.\mathrm{CDCl}_{3}\right) \delta$ 206.9, 176.8, 82.4, 57.2, 46.1, 45.1, 45.0, 44.7, 38.1, 19.5, 18.4; IR $v_{\max }$ 2969, 2928, 1779, 1726, 1192, 1152, 1054, 1031, 983 $\mathrm{cm}^{-1}$; MS (ESI, +ve) m/z $215\left[(\mathrm{M}+\mathrm{Na})^{+}, 100 \%\right.$; HRMS $(\mathrm{M}+\mathrm{Na})^{+}$calcd for $\mathrm{C}_{11} \mathrm{H}_{12} \mathrm{O}_{3}$ 215.0684, found 215.0687.

(1aS,1a'R,3aS,4S,4aS,4a'R)-4-Isopropyl-4a-methylhexahydro-1H-cyclopropa[3,4]pentaleno[1,6-bc]furan-1,3(1aH)-dione (29). A deoxygenated and magnetically stirred solution of compound 23 (123 mg, $0.56 \mathrm{mmol})$ and acetophenone (90 $\mu \mathrm{L}, 0.77$ mmol) in acetone $(100 \mathrm{~mL})$ maintained under a nitrogen atmosphere was subjected to irradiation with a Hanovia 450W medium pressure mercury-vapor lamp for $0.67 \mathrm{~h}$. The reaction mixture thus formed was cooled and concentrated under reduced pressure to give a yellow oil that was subjected to flash column chromatography 
(silica, 3:17 $\rightarrow$ 1:3 v/v ethyl acetate/40-60 petroleum ether gradient elution). Concentration of the appropriate fractions $\left(R_{\mathrm{f}}=0.4\right.$ in $1: 1 \mathrm{v} / \mathrm{v}$ ethyl acetate/40-60 petroleum ether) then gave compound 29 (53 mg, 43\%) as a white, crystalline solid, $\mathrm{mp}=107-109{ }^{\circ} \mathrm{C},[\alpha]_{\mathrm{D}}=-7.6\left(c\right.$ 1.0, $\left.\mathrm{CHCl}_{3}\right) .{ }^{1} \mathrm{H}$ NMR $\left(400 \mathrm{MHz}, \mathrm{CDCl}_{3}\right) \delta 4.60$ (dd, $J=8.9$ and $2.0 \mathrm{~Hz}, 1 \mathrm{H}$ ), $3.61(\mathrm{~m}, 1 \mathrm{H}), 3.10(\mathrm{dd}, J=8.5$ and $6.5 \mathrm{~Hz}, 1 \mathrm{H}), 2.62$ (m, 1H), 2.38 (m, 1H), 2.15 (m, 1H), 1.88 (m, 1H), 1.27 (s, 3H), 1.05 (d, $J=6.9 \mathrm{~Hz}, 3 \mathrm{H}$ ), $0.97(\mathrm{~d}, J=6.5 \mathrm{~Hz}, 3 \mathrm{H}) ;{ }^{13} \mathrm{C}$ NMR $\left(100 \mathrm{MHz}, \mathrm{CDCl}_{3}\right) \delta$ 206.9, 177.4, 82.5, 55.0, 47.8, 46.6, 45.6, 44.0, 38.3, 29.2, 22.1, 19.5, 17.0; IR $v_{\max } 2963,1780,1728,1196$,

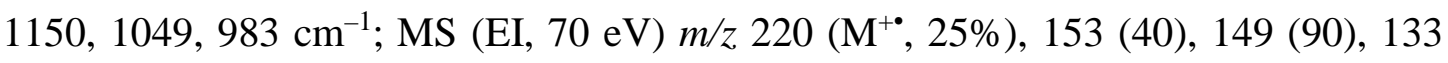
(40), 105 (60), 93 (100), 91 (70), 84 (60), 77 (50), 59 (70); HRMS M+• calcd for $\mathrm{C}_{13} \mathrm{H}_{16} \mathrm{O}_{3} 220.1099$, found 220.1092 .

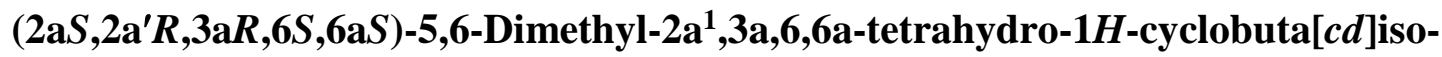
benzofuran-1,3(2aH)-dione (30). A deoxygenated and magnetically stirred solution of compound 22 (100 mg, $0.52 \mathrm{mmol})$ in dichloromethane (100 mL) maintained under a nitrogen atmosphere was subjected to irradiation with a Hanovia 450W medium pressure mercury-vapor lamp for $0.5 \mathrm{~h}$. The reaction thus obtained was then cooled and concentrated under reduced pressure to give a yellow solid. Subjection of this material to flash column chromatography (silica, 1:4 $\rightarrow 3: 7 \mathrm{v} / \mathrm{v}$ ethyl acetate/4060 petroleum ether gradient elution) gave, after concentration of the appropriate fractions $\left(R_{\mathrm{f}}=0.2\right.$ in 3:7 v/v ethyl acetate/40-60 petroleum ether), compound 30 (75 mg, 75\%) as a white, crystalline solid, $\mathrm{mp}=161-163{ }^{\circ} \mathrm{C},[\alpha]_{\mathrm{D}}=-344.2(\mathrm{c} 1.0$, $\left.\mathrm{CHCl}_{3}\right) .{ }^{1} \mathrm{H}$ NMR (400 MHz, $\left.\mathrm{CDCl}_{3}\right) \delta 5.37(\mathrm{~m}, 1 \mathrm{H}), 5.33(\mathrm{~m}, 1 \mathrm{H}), 3.93(\mathrm{~m}, 1 \mathrm{H})$, 3.48 (m, 1H), 2.97 (dd, $J=10.0$ and $2.1 \mathrm{~Hz}, 1 \mathrm{H}), 2.67$ (m, 1H), 1.78 (s, 3H), 1.19 (d, $J=7.2 \mathrm{~Hz}, 3 \mathrm{H}) ;{ }^{13} \mathrm{C}$ NMR $\left(100 \mathrm{MHz}, \mathrm{CDCl}_{3}\right) \delta 200.3,177.5,140.6,112.9,89.8$, 58.8, 43.3, 33.9, 26.8, 23.0, 19.8; IR $v_{\max }$ 2968, 1782, 1256, 1148, 1141, 1130, 1004 
$\mathrm{cm}^{-1}$; MS (ESI, +ve) m/z $247\left[(\mathrm{M}+\mathrm{MeOH}+\mathrm{Na})^{+}, 100 \%\right]$; HRMS $(\mathrm{M}+\mathrm{MeOH}+\mathrm{Na})^{+}$ calcd for $\mathrm{C}_{11} \mathrm{H}_{12} \mathrm{O}_{3} 247.0946$, found 247.0945.

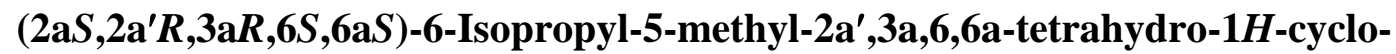

buta[cd]isobenzofuran-1,3(2aH)-dione (31). A deoxygenated and magnetically stirred solution of compound 23 (100 mg, $0.45 \mathrm{mmol})$ in dichloromethane (100 mL) maintained under nitrogen was subjected to irradiation with a Hanovia 450W medium pressure mercury-vapor lamp for $0.5 \mathrm{~h}$. The reaction mixture thus obtained was cooled and concentrated under reduced pressure to give a yellow oil. Subjection of this material to flash column chromatography (silica, 1:4 $\rightarrow 3: 7 \mathrm{v} / \mathrm{v}$ ethyl acetate/4060 petroleum ether gradient elution) gave, after concentration of the appropriate fractions $\left(R_{\mathrm{f}}=0.2\right.$ in 3:7 v/v ethyl acetate/40-60 petroleum ether), compound 31 (73 mg, 73\%) as a clear, colorless oil, $[\alpha]_{\mathrm{D}}=-163.3\left(\right.$ c $\left.0.7, \mathrm{CHCl}_{3}\right) .{ }^{1} \mathrm{H}$ NMR $(400 \mathrm{MHz}$, $\left.\mathrm{CDCl}_{3}\right) \delta 5.47$ (s, 1H), 5.27 (dd, $J=6.4$ and $\left.4.0 \mathrm{~Hz}, 1 \mathrm{H}\right), 3.88(\mathrm{~m}, 1 \mathrm{H}), 3.43(\mathrm{~m}, 1 \mathrm{H})$, 3.08 (dd, $J=10.1$ and $1.4 \mathrm{~Hz}, 1 \mathrm{H}$ ), 2.45 (d, $J=3.9 \mathrm{~Hz}, 1 \mathrm{H}$ ), 1.90 (m, 1H), 1.79 (s, 3H), $1.06(\mathrm{~d}, J=6.8 \mathrm{~Hz}, 3 \mathrm{H}), 0.89(\mathrm{~d}, J=6.9 \mathrm{~Hz}, 3 \mathrm{H}) ;{ }^{13} \mathrm{C} \mathrm{NMR}\left(100 \mathrm{MHz}, \mathrm{CDCl}_{3}\right)$ $\delta$ 200.4, 178.7, 138.9, 114.7, 89.1, 59.4, 45.9, 38.1, 30.8, 28.3, 24.5, 21.6, 19.6; IR $v_{\max }$ 2963, 1782, 1168, 1147, 1131, 1005, $838 \mathrm{~cm}^{-1}$; MS (ESI, +ve) m/z 275 $\left[(\mathrm{M}+\mathrm{MeOH}+\mathrm{Na})^{+}, 100 \%\right], 221\left[(\mathrm{M}+\mathrm{H})^{+}, 25\right]$; HRMS $(\mathrm{M}+\mathrm{MeOH}+\mathrm{Na})^{+}$calcd for $\mathrm{C}_{13} \mathrm{H}_{16} \mathrm{O}_{3} 275.1259$, found 275.1260.

\section{(2aS,2a'R,3S,5aR,5bR)-3,4-Dimethyl-2a' ,3,5a,5b-tetrahydrocyclopropa[cd]iso-}

benzofuran-2(2aH)-one (32). A deoxygenated and magnetically stirred solution of compound 22 (100 mg, $0.52 \mathrm{mmol})$ in dichloromethane $(100 \mathrm{~mL})$ maintained under a nitrogen atmosphere was irradiated with a Hanovia 450W medium pressure mercuryvapor lamp for $5 \mathrm{~h}$. The ensuing reaction mixture was cooled to ca. $22{ }^{\circ} \mathrm{C}$ then concentrated under reduced pressure to give a brown oil. Subjection of this material to 
flash column chromatography (silica, $1: 9 \rightarrow 3: 7 \mathrm{v} / \mathrm{v}$ ethyl acetate/40-60 petroleum ether gradient elution) gave two fractions, $\mathrm{A}$ and $\mathrm{B}$.

Concentration of fraction A $\left(R_{\mathrm{f}}=0.5\right.$ in 3:7 v/v ethyl acetate/40-60 petroleum ether) afforded compound 32 (32 mg, 37\%) as a colorless, crystalline solid, $\mathrm{mp}=$ 73$75{ }^{\circ} \mathrm{C},[\alpha]_{\mathrm{D}}=-102.8\left(c\right.$ 0.4, $\left.\mathrm{CHCl}_{3}\right) .{ }^{1} \mathrm{H}$ NMR $\left(400 \mathrm{MHz}, \mathrm{CDCl}_{3}\right) \delta 5.34(\mathrm{~s}, 1 \mathrm{H}), 4.33$ (dd, $J=6.3$ and $5.4 \mathrm{~Hz}, 1 \mathrm{H}$ ), 2.89 (dd, $J=7.7$ and $3.0 \mathrm{~Hz}, 1 \mathrm{H}$ ), 2.23 (m, 1H), 1.95 (m, 1H), $1.69(\mathrm{~s}, 3 \mathrm{H}), 1.46(\mathrm{~m}, 1 \mathrm{H}), 1.21$ (d, $J=7.0 \mathrm{~Hz}, 3 \mathrm{H}) ;{ }^{13} \mathrm{C}$ NMR (100 MHz, $\left.\mathrm{CDCl}_{3}\right) \delta 180.3,142.7,112.7,60.5,44.7,35.9,22.4,18.7,17.8,11.6$; IR $v_{\max } 2965$, 1773, 1453, 1150, 1086, 1074, 922, $794 \mathrm{~cm}^{-1}$; MS (ESI, +ve) m/z $187\left[(\mathrm{M}+\mathrm{Na})^{+}\right.$, 100\%]; HRMS (M+Na) ${ }^{+}$calcd for $\mathrm{C}_{10} \mathrm{H}_{12} \mathrm{O}_{2}$ 187.0735, found 187.0735.

Concentration of fraction B $\left(R_{\mathrm{f}}=0.2\right.$ in $3: 7 \mathrm{v} / \mathrm{v}$ ethyl acetate $/ 40-60$ petroleum ether) afforded compound 30 (28 mg, 28\%) as a white solid that was identical with an authentic sample.

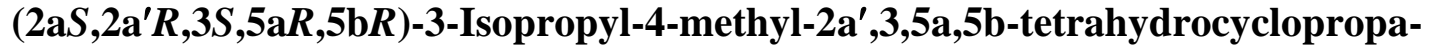

[cd]isobenzofuran-2(2aH)-one (33). The deoxygenated and magnetically stirred solution of compound 23 (100 mg, $0.45 \mathrm{mmol})$ in dichloromethane (100 mL) maintained under a nitrogen atmosphere was subjected to irradiation with a Hanovia 450W medium pressure mercury-vapor lamp for $1.5 \mathrm{~h}$. The ensuing mixture was cooled then concentrated under reduced pressure to give a brown oil. Subjection of this material to flash column chromatography (silica, $1: 9 \rightarrow 3: 7 \mathrm{v} / \mathrm{v}$ ethyl acetate/4060 petroleum ether gradient elution) afforded two fractions, A and B.

Concentration of fraction A $\left(R_{\mathrm{f}}=0.6\right.$ in 3:7 v/v ethyl acetate/40-60 petroleum ether) gave compound $33(45 \mathrm{mg}, 52 \%)$ as a clear, colorless oil, $[\alpha]_{\mathrm{D}}=-29.8(c$ 1.0, $\mathrm{CHCl}_{3}$ ). ${ }^{1} \mathrm{H}$ NMR (400 MHz, $\left.\mathrm{CDCl}_{3}\right) \delta 5.45$ (s, 1H), 4.33 (m, 1H), 3.03 (dd, $J=7.7$ and 2.4 Hz, 1H), 2.02-1.88 (complex m, 3H), 1.71 (s, 3H), 1.45 (m, 1H), 1.06 (d, $J=$ 
6.5 Hz, 3H), 0.98 (d, $J=6.6 \mathrm{~Hz}, 3 \mathrm{H}) ;{ }^{13} \mathrm{C} \mathrm{NMR}\left(100 \mathrm{MHz}, \mathrm{CDCl}_{3}\right) \delta$ 181.1, 141.1, 114.8, 61.4, 47.6, 40.3, 30.7, 24.2, 21.7, 19.9, 18.0, 13.5; IR $v_{\max } 2960,1769,1149$, 1127, 1087, 933, $781 \mathrm{~cm}^{-1}$; MS (ESI, +ve) m/z $215\left[(\mathrm{M}+\mathrm{Na})^{+}, 100 \%\right]$; HRMS $(\mathrm{M}+\mathrm{Na})^{+}$calcd for $\mathrm{C}_{12} \mathrm{H}_{16} \mathrm{O}_{2} 215.1048$, found 215.1048 .

Concentration of fraction B $\left(R_{\mathrm{f}}=0.2\right.$ in $3: 7 \mathrm{v} / \mathrm{v}$ ethyl acetate $/ 40-60$ petroleum ether) afforded compound 31 (25 mg, 25\%) as a white solid that was identical with an authentic sample.

\section{$\left(1 \mathrm{a} R, 1 \mathrm{a}^{\prime} S, 3 \mathrm{a} R, 4 S, 4 \mathrm{a}^{1} R\right)-1 \mathrm{a}^{\prime}, 4$-Dimethylhexahydro-1H-cyclopropa[3,4]pentaleno-}

[1,6-bc]furan-1,3(1aH)-dione (34). A deoxygenated and magnetically stirred solution of compound 26 (108 mg, $0.56 \mathrm{mmol})$ and acetophenone (90 $\mu \mathrm{L}, 0.77 \mathrm{mmol})$ in acetone $(100 \mathrm{~mL})$ maintained under nitrogen was subjected to irradiation with a Hanovia 450W medium pressure mercury-vapor lamp for $2.5 \mathrm{~h}$. The ensuing reaction mixture was cooled then concentrated under reduced pressure to give a yellow oil. Subjection of this material to flash column chromatography (silica, 3:17 $\rightarrow 3: 7 \mathrm{v} / \mathrm{v}$ ethyl acetate/40-60 petroleum ether gradient elution) gave, after concentration of the appropriate fractions $\left(R_{\mathrm{f}}=0.3\right.$ in $1: 1 \mathrm{v} / \mathrm{v}$ ethyl acetate/40-60 petroleum ether), compound 34 (49 mg, 45\%) as a white, crystalline solid, $\mathrm{mp}=154-156{ }^{\circ} \mathrm{C},[\alpha]_{\mathrm{D}}=$ $+68.6\left(\right.$ c 1.0, $\left.\mathrm{CHCl}_{3}\right) .{ }^{1} \mathrm{H}$ NMR $\left(400 \mathrm{MHz}, \mathrm{CDCl}_{3}\right) \delta 4.21$ (s, 1H), 2.55-2.47 (complex m, 3H), 2.18 (m, 1H), 1.97 (m, 1H), 1.54 (s, 3H), 1.38 (d, $J=6.8 \mathrm{~Hz}, 3 \mathrm{H}) ;{ }^{13} \mathrm{C}$ NMR $\left(100 \mathrm{MHz}, \mathrm{CDCl}_{3}\right) \delta$ 206.9, 176.5, 87.7, 64.3, 55.0, 42.6, 40.3, 38.0, 35.6, 22.6, 20.8; IR $v_{\max }$ 2970, 1781, 1726, 1238, 1163, 1033, 884, $874 \mathrm{~cm}^{-1}$; MS (ESI, +ve) $\mathrm{m} / \mathrm{z} 247$ $\left[(\mathrm{M}+\mathrm{MeOH}+\mathrm{Na})^{+}, 20 \%\right], 215\left[(\mathrm{M}+\mathrm{Na})^{+}, 100\right], 193(30)$; HRMS $(\mathrm{M}+\mathrm{Na})^{+}$calcd for $\mathrm{C}_{11} \mathrm{H}_{12} \mathrm{O}_{3} 215.0684$, found:215.0676.

(1aR,1a'S,3aR,4S,4a'R)-4-Isopropyl-1a'-methylhexahydro-1H-cyclopropa[3,4]pentaleno[1,6-bc]furan-1,3(1aH)-dione (35). A deoxygenated and magnetically 
stirred solution of compound $27(123 \mathrm{mg}, 0.56 \mathrm{mmol})$ and acetophenone (90 $\mu \mathrm{L}, 0.77$ mmol) in acetone $(100 \mathrm{~mL})$ maintained under a nitrogen atmosphere was irradiated with a Hanovia 450W medium pressure mercury-vapor lamp for $2.5 \mathrm{~h}$. The cooled reaction mixture was concentrated under reduced pressure to give a yellow oil and this subjected to flash column chromatography (silica, 3:17 $\rightarrow$ 1:3 v/v ethyl acetate/40-60 petroleum ether gradient elution). Concentration of the appropriate fractions $\left(R_{\mathrm{f}}=0.5\right.$ in $1: 1 \mathrm{v} / \mathrm{v}$ ethyl acetate/40-60 petroleum ether) then gave compound $35(60 \mathrm{mg}, 49 \%)$ as a white, crystalline solid, $\mathrm{mp}=102-103{ }^{\circ} \mathrm{C},[\alpha]_{\mathrm{D}}=$ +83.8 $\left(\right.$ c 1.0, $\left.\mathrm{CHCl}_{3}\right) .{ }^{1} \mathrm{H}$ NMR $\left(400 \mathrm{MHz}, \mathrm{CDCl}_{3}\right) \delta 4.22$ (s, 1H), $2.72(\mathrm{~d}, J=6.4 \mathrm{~Hz}$, 1H), 2.41 (t, $J=4.0 \mathrm{~Hz}, 1 \mathrm{H}), 2.22-2.15$ (complex m, 2H), 2.08 (m, 1H), $1.94(\mathrm{~m}, 1 \mathrm{H})$, 1.53 (s, 3H), 1.03 (d, $J=3.0 \mathrm{~Hz}, 3 \mathrm{H}), 1.02$ (d, $J=3.0 \mathrm{~Hz}, 3 \mathrm{H}) ;{ }^{13} \mathrm{C}$ NMR (100 MHz, $\left.\mathrm{CDCl}_{3}\right) \delta$ 207.1, 176.8, 87.6, 59.1, 54.6, 53.7, 37.4, 37.3, 35.5, 32.9, 20.6, 20.2, 19.4; IR $v_{\max }$ 2961, 1790, 1781, 1732, 1240, 1160, 1047, $889 \mathrm{~cm}^{-1}$; MS (ESI, +ve) m/z 275 $\left[(\mathrm{M}+\mathrm{MeOH}+\mathrm{Na})^{+}, 20 \%\right], 243\left[(\mathrm{M}+\mathrm{Na})^{+}, 100\right]$; HRMS $(\mathrm{M}+\mathrm{Na})^{+}$calcd for $\mathrm{C}_{13} \mathrm{H}_{16} \mathrm{O}_{3}$ 243.0997, found 243.0993.

(2aR,2a'S,3aS,6S,6aR)-2a',6-Dimethyl-2a',3a,6,6a-tetrahydro-1H-cyclobuta[cd]isobenzofuran-1,3(2aH)-dione (36). A deoxygenated and magnetically stirred solution of compound 26 (100 mg, $0.52 \mathrm{mmol})$ in dichloromethane (100 mL) maintained under a nitrogen atmosphere was irradiated with a Hanovia 450W medium pressure mercury-vapor lamp for $0.58 \mathrm{~h}$ then cooled and concentrated under reduced pressure to give a yellow solid. Subjection of this material to flash column chromatography (silica, 1:4 $\rightarrow$ 3:7 v/v ethyl acetate/40-60 petroleum ether gradient elution) then gave, after concentration of the appropriate fractions $\left(R_{\mathrm{f}}=0.2\right.$ in $3: 7 \mathrm{v} / \mathrm{v}$ ethyl acetate/40-60 petroleum ether), compound 36 (70 mg, 70\%) as a white, crystalline solid, $\mathrm{mp}=100-101{ }^{\circ} \mathrm{C},[\alpha]_{\mathrm{D}}=+220.5\left(\right.$ c 1.0, $\left.\mathrm{CHCl}_{3}\right) .{ }^{1} \mathrm{H}$ NMR $(400$ 
$\left.\mathrm{MHz}, \mathrm{CDCl}_{3}\right) \delta 6.02(\mathrm{~m}, 1 \mathrm{H}), 5.69(\mathrm{dd}, J=10.0$ and $4.0 \mathrm{~Hz}, 1 \mathrm{H}), 4.82(\mathrm{~d}, J=4.0 \mathrm{~Hz}$, 1H), 3.60 (m, 1H), 2.93 (m, 1H), 2.66 (s, 1H), 1.70 (s, 3H), 1.23 (d, J = 7.3 Hz, 3H); ${ }^{13} \mathrm{C}$ NMR $\left(100 \mathrm{MHz}, \mathrm{CDCl}_{3}\right) \delta 200.1,177.7,132.6,119.1,93.5,63.8,48.5,35.5$, 30.1, 24.9, 21.2; IR $v_{\max }$ 2965, 1779, 1191, 1096, 997, 744, $725 \mathrm{~cm}^{-1}$; MS (ESI, +ve) $m / z 247\left[(\mathrm{M}+\mathrm{MeOH}+\mathrm{Na})^{+}, 100 \%\right]$; HRMS $(\mathrm{M}+\mathrm{MeOH}+\mathrm{Na})^{+}$calcd for $\mathrm{C}_{11} \mathrm{H}_{12} \mathrm{O}_{3}$ 247.0946, found 247.0947.

$\left(2 \mathrm{a} R, 2 \mathrm{a}^{\prime} S, 3 \mathrm{a} S, 6 S, 6 \mathrm{a} R\right)-6-$ Isopropyl-2a'-methyl-2a',3a,6,6a-tetrahydro-1H-cyclobuta[cd]isobenzofuran-1,3(2aH)-dione (37). A deoxygenated and magnetically stirred solution of compound 27 (100 mg, $0.45 \mathrm{mmol})$ in dichloromethane (100 mL) maintained under a nitrogen atmosphere was irradiated with a Hanovia 450W medium pressure mercury-vapor lamp for $0.55 \mathrm{~h}$ then cooled and concentrated under reduced pressure to give a yellow solid. Subjection of this material to flash column chromatography (silica, 1:4 $\rightarrow$ 3:7 v/v ethyl acetate/40-60 petroleum ether gradient elution) then gave, after concentration of the appropriate fractions $\left(R_{\mathrm{f}}=0.2\right.$ in $3: 7 \mathrm{v} / \mathrm{v}$ ethyl acetate/40-60 petroleum ether), compound 37 (71 $\mathrm{mg}, 71 \%)$ as a white, crystalline solid, $\mathrm{mp}=96-98{ }^{\circ} \mathrm{C},[\alpha]_{\mathrm{D}}=+196.2\left(\mathrm{c} 1.0, \mathrm{CHCl}_{3}\right) .{ }^{1} \mathrm{H}$ NMR $(400 \mathrm{MHz}$, $\left.\mathrm{CDCl}_{3}\right) \delta 6.11(\mathrm{~m}, 1 \mathrm{H}), 5.73(\mathrm{dd}, J=10.1$ and $3.6 \mathrm{~Hz}, 1 \mathrm{H}), 4.79(\mathrm{~d}, J=3.6 \mathrm{~Hz}, 1 \mathrm{H})$, 3.56 (m, 1H), 2.95 (d, $J=1.7 \mathrm{~Hz}, 1 \mathrm{H}), 2.43$ (t, $J=8.0 \mathrm{~Hz}, 1 \mathrm{H}), 1.68$ (s, 3H), 1.661.60 (complex m, 1H), 1.09 (d, $J=6.6 \mathrm{~Hz}, 3 \mathrm{H}), 1.01$ (d, $J=6.6 \mathrm{~Hz}, 3 \mathrm{H}) ;{ }^{13} \mathrm{C} \mathrm{NMR}$ (100 MHz, $\left.\mathrm{CDCl}_{3}\right) \delta$ 199.9, 178.5, 131.8, 120.0, 93.5, 64.2, 44.8, 42.6, 35.9, 33.1, 24.1, 21.4, 21.3; IR $v_{\max }$ 2964, 1782, 1471, 1293, 1193, 1000, $722 \mathrm{~cm}^{-1}$; MS (ESI, +ve) $m / z 527\left[(2 \mathrm{M}+2 \mathrm{MeOH}+\mathrm{Na})^{+}, 15 \%\right], 275\left[(\mathrm{M}+\mathrm{MeOH}+\mathrm{Na})^{+}, 100\right], 221\left[(\mathrm{M}+\mathrm{H})^{+}\right.$, 15]; HRMS (M+MeOH+Na) ${ }^{+}$calcd for $\mathrm{C}_{13} \mathrm{H}_{16} \mathrm{O}_{3} 275.1259$, found 275.1257.

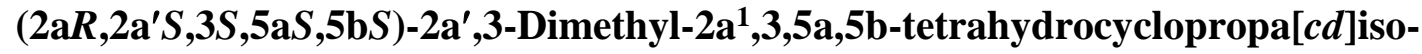
benzofuran-2(2aH)-one (38). A deoxygenated and magnetically stirred solution of 
compound 26 (100 mg, $0.52 \mathrm{mmol})$ in dichloromethane (100 mL) maintained under a nitrogen atmosphere was irradiated with a Hanovia 450W medium pressure mercuryvapor lamp for $3.1 \mathrm{~h}$ then cooled and concentrated under reduced pressure to give a brown oil. Subjection of this material to flash column chromatography (silica, 1:9 $\rightarrow$ 3:7 v/v ethyl acetate/40-60 petroleum ether gradient elution) then gave two fractions, A and B.

Concentration of fraction $\mathrm{A}\left(R_{\mathrm{f}}=0.5\right.$ in $3: 7 \mathrm{v} / \mathrm{v}$ ethyl acetate $/ 40-60$ petroleum ether) afforded compound 38 (30 mg, 35\%) as a colorless, crystalline solid, $\mathrm{mp}=$ 40$42{ }^{\circ} \mathrm{C},[\alpha]_{\mathrm{D}}=+152.0\left(c \mathrm{c} 0.5, \mathrm{CHCl}_{3}\right) .{ }^{1} \mathrm{H}$ NMR $\left(400 \mathrm{MHz}, \mathrm{CDCl}_{3}\right) \delta 5.95$ (dd, $J=9.9$ and $6.0 \mathrm{~Hz}, 1 \mathrm{H}$ ), 5.66 (dd, $J=9.9$ and $4.5 \mathrm{~Hz}, 1 \mathrm{H}), 4.03$ (d, $J=5.6 \mathrm{~Hz}, 1 \mathrm{H}), 2.65$ (s, 1H), 2.54 (m, 1H), 1.39 (s, 3H), 1.35 (m, 1H), 1.22 (d, $J=7.1 \mathrm{~Hz}, 3 \mathrm{H}) ;{ }^{13} \mathrm{C}$ NMR $\left(100 \mathrm{MHz}, \mathrm{CDCl}_{3}\right) \delta 180.7,134.8,119.4,65.2,49.3,31.7,23.5,20.6,19.3,18.9 ; \mathrm{IR}$ $v_{\max }$ 2962, 2929, 1783, 1769, 1143, 1090, 846, $750 \mathrm{~cm}^{-1}$; MS (EI, $\left.70 \mathrm{eV}\right) \mathrm{m} / \mathrm{z} 164$ (M+•, 20\%), 121 (30), 107 (100), 91 (70), 77 (25), 65 (15); HRMS M+• calcd for $\mathrm{C}_{10} \mathrm{H}_{12} \mathrm{O}_{2}$ 164.0837, found 164.0835.

Concentration of fraction B $\left(R_{\mathrm{f}}=0.2\right.$ in $3: 7 \mathrm{v} / \mathrm{v}$ ethyl acetate $/ 40-60$ petroleum ether) afforded compound 36 (32 mg, 32\%) as a white, crystalline solid that was identical, in all respects, with an authentic sample.

\section{(2aR,2a'S,3S,5aS,5bS)-3-Isopropyl-2a'-methyl-2a',3,5a,5b-tetrahydrocyclopropa}

-[cd]isobenzofuran-2(2aH)-one (39). A deoxygenated and magnetically stirred solution of compound 27 (100 $\mathrm{mg}, 0.45 \mathrm{mmol})$ in dichloromethane (100 mL) maintained under a nitrogen atmosphere was irradiated with a Hanovia 450W medium pressure mercury-vapor lamp for $3.1 \mathrm{~h}$ then cooled and concentrated under reduced pressure to give a brown oil. Subjection of this material to flash column chromatography (silica, 1:9 $\rightarrow$ 3:7 v/v ethyl acetate/40-60 petroleum ether elution) 
then gave two fractions, $\mathrm{A}$ and $\mathrm{B}$.

Concentration of fraction A $\left(R_{\mathrm{f}}=0.6\right.$ in 3:7 v/v ethyl acetate/40-60 petroleum ether) afforded compound 39 (35 mg, 40\%) as a yellow oil, $[\alpha]_{\mathrm{D}}=+166.7$ (c 1.0, $\mathrm{CHCl}_{3}$ ). ${ }^{1} \mathrm{H}$ NMR (400 MHz, $\left.\mathrm{CDCl}_{3}\right) \delta 6.02$ (dd, $J=9.9$ and $6.1 \mathrm{~Hz}, 1 \mathrm{H}$ ), 5.70 (dd, $J$ = 9.9 and $4.2 \mathrm{~Hz}, 1 \mathrm{H}), 4.02(\mathrm{~d}, J=5.5 \mathrm{~Hz}, 1 \mathrm{H}), 2.92(\mathrm{~s}, 1 \mathrm{H}), 2.02(\mathrm{~m}, 1 \mathrm{H}), 1.79(\mathrm{~m}$, 1H), 1.36 (s, 3H), 1.34 (m, 1H), 1.05 (d, $J=6.6 \mathrm{~Hz}, 3 \mathrm{H}), 0.99$ (d, $J=6.6 \mathrm{~Hz}, 3 \mathrm{H}) ;{ }^{13} \mathrm{C}$ NMR $\left(100 \mathrm{MHz}, \mathrm{CDCl}_{3}\right) \delta 181.2,134.0,120.3,65.5,46.0,43.8,32.3,23.7,21.8$, 20.9, 20.1, 19.3; IR $v_{\max }$ 2966, 2872, 1778, 1148, 1092, 834, $752 \mathrm{~cm}^{-1}$; MS (ESI, +ve) m/z $215\left[(\mathrm{M}+\mathrm{Na})^{+}, 100 \%\right]$; HRMS $(\mathrm{M}+\mathrm{Na})^{+}$calcd for $\mathrm{C}_{12} \mathrm{H}_{16} \mathrm{O}_{2} 215.1048$, found 215.1047.

Concentration of fraction B $\left(R_{\mathrm{f}}=0.2\right.$ in 3:7 v/v ethyl acetate $/ 40-60$ petroleum ether) afforded compound 37 (34 mg, 34\%) as a white, crystalline solid that was identical, in all respects, with an authentic sample.

(3aR,4R,4aR,5S,7aR,8S,8aS)-2,2,4,6,6-Pentamethyl-3a,4a,5,6,7,7a,8,8a-octahydro -4H-4,8-ethenoindeno[5,6-d][1,3]dioxol-5-yl Acetate (42). A magnetically stirred solution of compound $\mathbf{4 1} \mathbf{1}^{4 \mathrm{~d}, 5 \mathrm{~b}}$ (5.00 g, $17.96 \mathrm{mmol}$ ), acetic anhydride (3.40 mL, 35.97 mmol) and 4-(dimethylamino)pyridine (440 mg, $3.60 \mathrm{mmol})$ in triethylamine (7.50 $\mathrm{mL}, 53.8 \mathrm{mmol}$ ) was stirred at $0{ }^{\circ} \mathrm{C}$ for $2 \mathrm{~h}$ then warmed to $22{ }^{\circ} \mathrm{C}$ and stirred at this temperature for another $2 \mathrm{~h}$. After this time $\mathrm{NH}_{4} \mathrm{Cl}(50 \mathrm{~mL}$ of a saturated aqueous solution) was added to the reaction mixture and the resulting suspension was extracted with dichloromethane $(3 \times 50 \mathrm{~mL})$. The combined organic fractions were washed with water $(2 \times 50 \mathrm{~mL})$ and brine $(1 \times 50 \mathrm{~mL})$ before being dried $\left(\mathrm{MgSO}_{4}\right)$, filtered, and concentrated under reduced pressure. The residue thus obtained was subjected to flash column chromatography (silica, 1:19 v/v ethyl acetate/40-60 petroleum ether elution) and gave, after concentration of the appropriate fractions $\left(R_{\mathrm{f}}\right.$ 
$=0.3$ in 1:9 v/v ethyl acetate/40-60 petroleum ether), compound 42 (5.58 g, 97\%) as a white, crystalline solid, mp $=135-137{ }^{\circ} \mathrm{C},[\alpha]_{\mathrm{D}}=+7.6\left(\right.$ c 1.0, $\left.\mathrm{CHCl}_{3}\right) .{ }^{1} \mathrm{H}$ NMR $(400$ $\left.\mathrm{MHz}, \mathrm{CDCl}_{3}\right) \delta$ 5.86-5.80 (complex m, 2H), $4.98(\mathrm{~d}, J=6.1 \mathrm{~Hz}, 1 \mathrm{H}), 4.22(\mathrm{dd}, J=$ 7.2 and $3.1 \mathrm{~Hz}, 1 \mathrm{H}), 3.79$ (d, $J=7.2 \mathrm{~Hz}, 1 \mathrm{H}), 2.75$ (m, 1H), 2.22 (m, 1H), 2.07 (m, 1H), 2.04 (s, 3H), 1.37 (m, 2H), 1.32 (s, 3H), 1.27 (s, 3H), 1.14 (s, 3H), 0.95 (s, 3H), 0.82 (s, 3H); ${ }^{13} \mathrm{C}$ NMR (100 MHz, $\left.\mathrm{CDCl}_{3}\right) \delta 170.4,137.3,125.7,109.1,84.1,81.0$, 80.1, 49.7, 44.7, 42.0, 40.8, 40.0, 38.0, 25.6(5), 25.6(2), 25.2, 22.4, 21.6, 19.9; IR $v_{\max }$ 2961, 2934, 1733, 1370, 1234, 1073, $723 \mathrm{~cm}^{-1}$; MS (ESI, +ve) m/z $343\left[(\mathrm{M}+\mathrm{Na})^{+}\right.$, 100\%]; HRMS (M+Na) ${ }^{+}$calcd for $\mathrm{C}_{19} \mathrm{H}_{28} \mathrm{O}_{4} 343.1885$, found 343.1884.

\section{(3S,3aR,4R,7S,7aR,8S,9R)-8,9-Dihydroxy-2,2,4-trimethyl-2,3,3a,4,7,7a-hexahyd-}

ro-1H-4,7-ethanoinden-3-yl Acetate (43). AG-50W-X8 resin (3.32 g of $\mathrm{H}^{+}$form) was added to a magnetically stirred solution of compound 42 in methanol/water (72 $\mathrm{mL}$ of a 5:1 v/v mixture) and the resulting suspension heated under reflux for $67 \mathrm{~h}$ then cooled to $22{ }^{\circ} \mathrm{C}$ before filtered through a sintered glass funnel. The filtrate was concentrated under reduced pressure and the residue thus obtained subjected to flash column chromatography (silica, 3:7 v/v ethyl acetate/40-60 petroleum ether elution). Concentration of the appropriate fractions $\left(R_{\mathrm{f}}=0.1\right.$ in $3: 7 \mathrm{v} / \mathrm{v}$ ethyl acetate/40-60 petroleum ether) gave compound $43(1.80 \mathrm{~g}, 97 \%)$ as a white solid, $\mathrm{mp}=73-74{ }^{\circ} \mathrm{C}$, $[\alpha]_{\mathrm{D}}=-2.3\left(\right.$ c 1.1, $\left.\mathrm{CHCl}_{3}\right) .{ }^{1} \mathrm{H}$ NMR $\left(400 \mathrm{MHz}, \mathrm{CDCl}_{3}\right) \delta$ 5.99-5.91 (complex m, 2H), 4.97 (d, $J=6.1 \mathrm{~Hz}, 1 \mathrm{H}$ ), 3.88 (dd, $J=7.5$ and $2.4 \mathrm{~Hz}, 1 \mathrm{H}$ ), 3.40 (d, $J=7.5 \mathrm{~Hz}$, 1H), 2.73 (m, 1H), 2.52 (broad s, 2H), 2.27 (m, 1H), 2.13 (m, 1H), 2.03 (s, 3H), 1.39 (m, 1H), 1.25 (t, $J=11.6 \mathrm{~Hz}, 1 \mathrm{H}), 1.15$ (s, 3H), 0.93 (s, 3H), 0.80 (s, 3H); ${ }^{13} \mathrm{C}$ NMR $\left(100 \mathrm{MHz}, \mathrm{CDCl}_{3}\right) \delta 170.4,138.8,126.8,81.6,75.4,71.7,50.4,44.1,42.0,41.5,41.3$, 40.8, 25.5, 22.3, 21.6, 19.6; IR $v_{\max }$ 3394, 2965, 2932, 1732, 1374, 1240, $1057 \mathrm{~cm}^{-1}$; 
MS m/z $583\left[(2 \mathrm{M}+\mathrm{Na})^{+}, 5 \%\right], 303\left[(\mathrm{M}+\mathrm{Na})^{+}, 100\right]$; HRMS $(\mathrm{M}+\mathrm{Na})^{+}$calcd for $\mathrm{C}_{16} \mathrm{H}_{24} \mathrm{O}_{4}$ 303.1572, found 303.1577.

(3S,3aR,4R,7S,7aS,9R)-9-Hydroxy-2,2,4-trimethyl-8-oxo-2,3,3a,4,7,7a-hexahydro -1H-4,7-ethanoinden-3-yl Acetate (44). A magnetically stirred mixture of $p$ toluenesulfonic acid monohydrate (448 mg, $2.36 \mathrm{mmol}$ ) and 4-acetamido-TEMPO (502 mg, $2.35 \mathrm{mmol})$ in dichloromethane $(10 \mathrm{~mL})$ was maintained at $0{ }^{\circ} \mathrm{C}$ under a nitrogen atmosphere for $0.5 \mathrm{~h}$ then added, dropwise over $2 \mathrm{~h}$, to a magnetically stirred solution of compound 43 (300 mg, $1.07 \mathrm{mmol})$ in dichloromethane (10 mL) maintained at $0{ }^{\circ} \mathrm{C}$. The ensuing mixture was stirred for a further $2 \mathrm{~h}$ at this temperature then quenched with $\mathrm{NaHCO}_{3}$ ( $50 \mathrm{~mL}$ of a saturated aqueous solution). The resulting suspension was extracted with dichloromethane $(3 \times 50 \mathrm{~mL})$ and the combined organic phases were washed with water $(2 \times 50 \mathrm{~mL})$ and brine $(1 \times 50 \mathrm{~mL})$ before being dried $\left(\mathrm{Na}_{2} \mathrm{SO}_{4}\right)$, filtered, and concentrated under reduced pressure. The residue thus obtained was subjected to flash column chromatography (silica, 3:17 v/v ethyl acetate/40-60 petroleum ether elution) to give, after concentration of the appropriate fractions $\left(R_{\mathrm{f}}=0.2\right.$ in $3: 7 \mathrm{v} / \mathrm{v}$ ethyl acetate/40-60 petroleum ether), compound $44(289 \mathrm{mg}, 97 \%)$ as a white, crystalline solid, $\mathrm{mp}=147-149{ }^{\circ} \mathrm{C},[\alpha]_{\mathrm{D}}=$ +215.0 (c 1.1, $\left.\mathrm{CHCl}_{3}\right) .{ }^{1} \mathrm{H} \mathrm{NMR}\left(400 \mathrm{MHz}, \mathrm{CDCl}_{3}\right) \delta 6.08$ (d, $\left.J=7.9 \mathrm{~Hz}, 1 \mathrm{H}\right), 5.92$ (t, $J=7.9 \mathrm{~Hz}, 1 \mathrm{H}), 5.09$ (d, $J=6.0 \mathrm{~Hz}, 1 \mathrm{H}), 3.34$ (s, 1H), 3.15 (d, $J=6.0 \mathrm{~Hz}, 1 \mathrm{H})$, 2.72-2.49 (complex m, 3H), 2.06 (s, 3H), 1.52 (dd, $J=12.2$ and $7.4 \mathrm{~Hz}, 1 \mathrm{H}), 1.40$ (t, $J$ $=12.2 \mathrm{~Hz}, 1 \mathrm{H}), 1.22(\mathrm{~s}, 3 \mathrm{H}), 1.00(\mathrm{~s}, 3 \mathrm{H}), 0.87$ (s, 3H); ${ }^{13} \mathrm{C} \mathrm{NMR}\left(100 \mathrm{MHz}, \mathrm{CDCl}_{3}\right)$ $\delta 211.0,170.2,142.2,121.9,81.1,75.2,51.8,50.1,44.8,43.9,41.7,39.3,25.6,22.2$, 21.6, 18.1; IR $v_{\max }$ 3451, 3239, 2965, 1730, 1374, 1236, 1081, $1039 \mathrm{~cm}^{-1}$; MS (ESI, +ve) $\mathrm{m} / \mathrm{z} 579\left[(2 \mathrm{M}+\mathrm{Na})^{+}, 10 \%\right], 301\left[(\mathrm{M}+\mathrm{Na})^{+}, 100\right]$; HRMS $(\mathrm{M}+\mathrm{Na})^{+}$calcd for $\mathrm{C}_{16} \mathrm{H}_{22} \mathrm{O}_{4}$ 301.1416, found 301.1411. 
(3S,3aR,4R,7S,7aS,9R)-2,2,4-Trimethyl-8-oxo-2,3,3a,4,7,7a-hexahydro-1H-4,7-eth -anoindene-3,9-diyl Diacetate (45). A magnetically stirred solution of compound 44 (239 mg, $0.86 \mathrm{mmol})$, 4-(dimethylamino)pyridine (11 mg, $0.09 \mathrm{mmol}$ ) and triethylamine (180 uL, $1.29 \mathrm{mmol})$ in dichloromethane $(10 \mathrm{~mL})$ maintained at $0{ }^{\circ} \mathrm{C}$ under a nitrogen atmosphere was treated with acetic anhydride (97 uL, $1.03 \mathrm{mmol})$. The ensuing mixture was kept at $0{ }^{\circ} \mathrm{C}$ for $2 \mathrm{~h}$ then at $22{ }^{\circ} \mathrm{C}$ for $16 \mathrm{~h}$ before being quenched with $\mathrm{NH}_{4} \mathrm{Cl}$ (10 mL of a saturated aqueous solution). The resulting mixture was extracted with dichloromethane $(2 \times 20 \mathrm{~mL})$ and the combined organic phases were washed with water $(2 \times 20 \mathrm{~mL})$ and brine $(1 \times 20 \mathrm{~mL})$ before being dried ( $\left.\mathrm{Na}_{2} \mathrm{SO}_{4}\right)$, filtered and concentrated under reduced pressure. The residue thus obtained was subjected to flash column chromatography (silica, 3:17 v/v ethyl acetate/40-60 petroleum ether elution) and gave, after concentration of the appropriate fractions $\left(R_{\mathrm{f}}\right.$

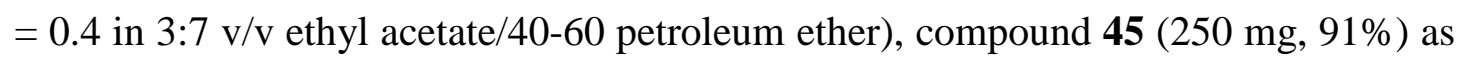
a white, crystalline solid, $\mathrm{mp}=175-177^{\circ} \mathrm{C},[\alpha]_{\mathrm{D}}=+200.0\left(\right.$ c 1.0, $\left.\mathrm{CHCl}_{3}\right) .{ }^{1} \mathrm{H}$ NMR $\left(400 \mathrm{MHz}, \mathrm{CDCl}_{3}\right) \delta 6.09(\mathrm{~d}, J=8.1 \mathrm{~Hz}, 1 \mathrm{H}), 5.97(\mathrm{t}, J=8.1 \mathrm{~Hz}, 1 \mathrm{H}), 5.09(\mathrm{~d}, J=$ $6.0 \mathrm{~Hz}, 1 \mathrm{H}$ ), 4.85 (s, 1H), 3.17 (d, $J=6.0 \mathrm{~Hz}, 1 \mathrm{H}), 2.74$ (m, 1H), 2.64 (dd, $J=10.6$ and $6.0 \mathrm{~Hz}, 1 \mathrm{H}), 2.10$ (s, 3H), 2.06 (s, 3H), 1.53 (dd, $J=12.2$ and $7.3 \mathrm{~Hz}, 1 \mathrm{H}), 1.39$ (t, $J=12.2 \mathrm{~Hz}, 1 \mathrm{H}), 1.05$ (s, 3H), 1.01 (s, 3H), 0.86 (s, 3H); ${ }^{13} \mathrm{C}$ NMR (100 MHz, $\left.\mathrm{CDCl}_{3}\right) \delta$ 205.2, 170.7, 170.1, 141.4, 122.2, 81.1, 74.2, 51.5, 50.7, 44.9, 42.6, 41.7, 39.6, 25.5, 22.2, 21.5, 20.7, 17.9; IR $v_{\max }$ 2979, 2965, 2932, 1721, 1374, 1230, 1053, $1020 \mathrm{~cm}^{-1}$; MS (ESI, +ve) m/z $663\left[(2 \mathrm{M}+\mathrm{Na})^{+}, 20 \%\right], 343\left[(\mathrm{M}+\mathrm{Na})^{+}, 100\right], 321$ $\left[(\mathrm{M}+\mathrm{H})^{+}, 5\right]$; HRMS $(\mathrm{M}+\mathrm{Na})^{+}$calcd for $\mathrm{C}_{18} \mathrm{H}_{24} \mathrm{O}_{5}$ 343.1521, found 343.1505 .

(3S,3aR,4R,7S,7aS,9R)-9-(Methoxymethoxy)-2,2,4-trimethyl-8-oxo-2,3,3a,4,7,7ahexahydro-1H-4,7-ethanoinden-3-yl Acetate (46). A magnetically stirred solution of compound 44 (280 mg, $1.00 \mathrm{mmol}$ ), chloromethyl methyl ether (122 uL, 1.60 
mmol), tetra- $n$-butylammonium iodide $(37 \mathrm{mg}, 0.10 \mathrm{mmol})$ and $N, N$ diisopropylethylamine (348 uL, $2.00 \mathrm{mmol})$ in dichloromethane $(5 \mathrm{~mL})$ maintained under a nitrogen atmosphere was stirred at $22{ }^{\circ} \mathrm{C}$ for $48 \mathrm{~h}$ then treated with $\mathrm{NH}_{4} \mathrm{Cl}(10$ $\mathrm{mL}$ of a saturated aqueous solution) and extracted with dichloromethane $(2 \times 20 \mathrm{~mL})$. The combined organic phases were washed with water $(2 \times 20 \mathrm{~mL})$ and brine $(1 \times 20$ $\mathrm{mL})$ before being dried $\left(\mathrm{Na}_{2} \mathrm{SO}_{4}\right)$, filtered and concentrated under reduced pressure. The residue thus obtained was subjected to flash column chromatography (silica, 1:4 $\mathrm{v} / \mathrm{v}$ ethyl acetate/40-60 petroleum ether elution) and gave, after concentration of the appropriate fractions $\left(R_{\mathrm{f}}=0.3\right.$ in $1: 4 \mathrm{v} / \mathrm{v}$ ethyl acetate/40-60 petroleum ether), compound 46 (251 mg, 77\%) as a pale-yellow oil, $[\alpha]_{\mathrm{D}}=+213.0$ (c 1.0, $\left.\mathrm{CHCl}_{3}\right) .{ }^{1} \mathrm{H}$ NMR (400 MHz, $\left.\mathrm{CDCl}_{3}\right) \delta 6.06(\mathrm{~d}, J=8.0 \mathrm{~Hz}, 1 \mathrm{H}), 5.90$ (t, $\left.J=8.0 \mathrm{~Hz}, 1 \mathrm{H}\right), 5.03$ (d, $J=6.7 \mathrm{~Hz}, 2 \mathrm{H}), 4.59$ (d, $J=6.7 \mathrm{~Hz}, 1 \mathrm{H}), 3.38$ (s, 3H), 3.31 (s, 1H), 3.04 (d, $J=6.3$ Hz, 1H), 2.61 (m, 1H), 2.46 (dd, $J=10.6$ and $6.1 \mathrm{~Hz}, 1 \mathrm{H}), 2.03$ (s, 3H), 1.46 (dd, $J=$ 12.1 and $7.4 \mathrm{~Hz}, 1 \mathrm{H}), 1.35$ (t, $J=12.1 \mathrm{~Hz}, 1 \mathrm{H}), 1.14$ (s, 3H), 0.95 (s, 3H), 0.82 (s, 3H); ${ }^{13} \mathrm{C}$ NMR (100 MHz, $\left.\mathrm{CDCl}_{3}\right) \delta 209.3,170.1,141.0,121.5,97.5,81.1,77.1,56.3$, 51.0, 50.7, 44.8, 43.2, 41.5, 40.2, 25.3, 22.1, 21.5, 18.4; IR $v_{\max } 2966,1731,1372$, 1239, 1150, 1035, $710 \mathrm{~cm}^{-1}$; MS (ESI, +ve) m/z $667\left[(2 \mathrm{M}+\mathrm{Na})^{+}, 5 \%\right], 345\left[(\mathrm{M}+\mathrm{Na})^{+}\right.$, 100]; HRMS (M+Na) ${ }^{+}$calcd for $\mathrm{C}_{18} \mathrm{H}_{26} \mathrm{O}_{5} 345.1678$, found 345.1665 .

(3S,3aR,4R,7S,7aS,9R)-9-((tert-Butyldimethylsilyl)oxy)-2,2,4-trimethyl-8-oxo-2,3, -3a,4,7,7a-hexahydro-1H-4,7-ethanoinden-3-yl Acetate (47). A magnetically solution of compound 44 (400 mg, 1.44 mmol), tert-butyldimethylsilyl chloride (1.73 g, $11.48 \mathrm{mmol}$ ) and imidazole (980 mg, $14.39 \mathrm{mmol}$ ) in 1,2-dichloroethane (20 mL) kept under a nitrogen atmosphere was heated at reflux for $65 \mathrm{~h}$ then cooled, quenched with $\mathrm{NH}_{4} \mathrm{Cl}(10 \mathrm{~mL}$ of a saturated aqueous solution) and extracted with dichloromethane $(2 \times 40 \mathrm{~mL})$. The combined organic phases were washed with water 
$(2 \times 40 \mathrm{~mL})$ and brine $(1 \times 40 \mathrm{~mL})$ before being dried $\left(\mathrm{Na}_{2} \mathrm{SO}_{4}\right)$, filtered and concentrated under reduced pressure. The residue thus obtained was subjected to flash column chromatography (silica, 2:5:100 v/v/v ethyl acetate/dichloromethane/40-60 petroleum ether elution) and gave, after concentration of the appropriate fractions $\left(R_{\mathrm{f}}\right.$ $=0.6$ in 3:7 v/v ethyl acetate/40-60 petroleum ether), compound 47 (465 mg, 82\%) as a white, crystalline solid, $\mathrm{mp}=71-72{ }^{\circ} \mathrm{C},[\alpha]_{\mathrm{D}}=+121.0\left(\right.$ c 1.0, $\left.\mathrm{CHCl}_{3}\right) .{ }^{1} \mathrm{H}$ NMR $(400$ $\mathrm{MHz}_{\mathrm{CDCl}}$ ) $\delta 6.03(\mathrm{~d}, J=8.0 \mathrm{~Hz}, 1 \mathrm{H}), 5.87(\mathrm{t}, J=8.0 \mathrm{~Hz}, 1 \mathrm{H}), 5.06$ (d, $J=6.0 \mathrm{~Hz}$, 1H), 3.27 (s, 1H), 3.02 (dd, $J=6.0$ and $1.8 \mathrm{~Hz}, 1 \mathrm{H}$ ), 2.62 (m, 1H), 2.42 (dd, $J=10.6$ and 6.0 Hz, 1H), 2.06 (s, 3H), 1.51-1.37 (complex m, 2H), 1.12 (s, 3H), 0.99 (s, 3H), 0.86 (s, 12H), 0.10 (s, 3H), 0.09 (s, 3H); ${ }^{13} \mathrm{C}$ NMR (100 MHz, $\left.\mathrm{CDCl}_{3}\right) \delta$ 209.5, 170.3, 141.7, 120.9, 81.4, 76.0, 51.1, 50.5, 44.9, 44.4, 41.7, 40.3, 26.0, 25.5, 22.3, 21.6, 19.0, 18.6, -4.0, -5.0; IR $v_{\max } 2959,2930,2856,1733,1237,1129,1112,1093,858,837$, 778, $709 \mathrm{~cm}^{-1}$; MS m/z $415\left[(\mathrm{M}+\mathrm{Na})^{+}, 100 \%\right]$; HRMS $(\mathrm{M}+\mathrm{Na})^{+}$calcd for $\mathrm{C}_{22} \mathrm{H}_{36} \mathrm{O}_{4} \mathrm{Si}$ 415.2281, found 415.2275 .

(3S,3aR,4R,7S,7aS,9R)-3-Acetoxy-2,2,4-trimethyl-8-oxo-2,3,3a,4,7,7a-hexahydro-

1H-4,7-ethanoinden-9-yl Benzoate (48). A magnetically stirred solution of compound 44 (2.90 g, $10.42 \mathrm{mmol})$, 4-(dimethylamino)pyridine (127 mg, $1.04 \mathrm{mmol}$ ) and triethylamine $(2.9 \mathrm{~mL}, 21.00 \mathrm{mmol})$ in dichloromethane $(50 \mathrm{~mL})$ maintained under a nitrogen atmosphere at $0{ }^{\circ} \mathrm{C}$ was treated with benzoyl chloride $(1.50 \mathrm{~mL}$, $12.50 \mathrm{mmol})$. The ensuing mixture was allowed to warm to and then stirred at $22{ }^{\circ} \mathrm{C}$ for $16 \mathrm{~h}$ before being quenched with $\mathrm{NH}_{4} \mathrm{Cl}$ (50 $\mathrm{mL}$ of a saturated aqueous solution) then extracted with dichloromethane $(2 \times 100 \mathrm{~mL})$. The combined organic phases were washed with water $(2 \times 100 \mathrm{~mL})$ then brine $(1 \times 100 \mathrm{~mL})$ before being dried ( $\left.\mathrm{Na}_{2} \mathrm{SO}_{4}\right)$, filtered and concentrated under reduced pressure. The residue thus obtained was subjected to flash column chromatography (silica, 2:5:100 v/v/v ethyl 
acetate/dichloromethane/40-60 petroleum ether elution) and gave, after concentration of the appropriate fractions $\left[R_{\mathrm{f}}=0.2(5)\right.$ in $1: 19 \mathrm{v} / \mathrm{v}$ ethyl acetate $/ 40-60$ petroleum ether], compound 48 (3.69 g, 93\%) as a white, crystalline solid, mp $=134-136{ }^{\circ} \mathrm{C}$, $[\alpha]_{\mathrm{D}}=+196.0\left(\right.$ ( $\left.1.1, \mathrm{CHCl}_{3}\right) .{ }^{1} \mathrm{H} \mathrm{NMR}\left(400 \mathrm{MHz}, \mathrm{CDCl}_{3}\right) \delta 7.99(\mathrm{~d}, J=7.3 \mathrm{~Hz}, 2 \mathrm{H})$, 7.54 (t, $J=7.3 \mathrm{~Hz}, 1 \mathrm{H}), 7.41$ (t, $J=7.3 \mathrm{~Hz}, 2 \mathrm{H}), 6.20$ (d, $J=8.1 \mathrm{~Hz}, 1 \mathrm{H}), 6.04$ (t, $J=$ 8.1 Hz, 1H), 5.12-5.10 (complex m, 2H), 3.23 (dd, $J=6.6$ and $1.6 \mathrm{~Hz}, 1 \mathrm{H}), 2.80$ (m, 1H), 2.72 (dd, $J=10.6$ and $6.6 \mathrm{~Hz}, 1 \mathrm{H}$ ), 2.07 (s, 3H), 1.55 (dd, $J=12.1$ and $7.3 \mathrm{~Hz}$, 1H), 1.43 (m, 1H), 1.11 (s, 3H), 1.02 (s, 3H), 0.87 (s, 3H); ${ }^{13} \mathrm{C}$ NMR (100 MHz, $\left.\mathrm{CDCl}_{3}\right) \delta 205.0,170.1,166.2,141.3,133.4,130.0,129.5,128.5,122.4,81.1,74.4$, 51.3, 50.7, 44.9, 42.9, 41.7, 39.7, 25.4, 22.2, 21.5, 18.0; IR $v_{\max } 2967,1722,1268$, 1238, 1108, 729, $706 \mathrm{~cm}^{-1}$; MS (ESI, +ve) m/z $787\left[(2 \mathrm{M}+\mathrm{Na})^{+}, 100 \%\right], 405$ $\left[(\mathrm{M}+\mathrm{Na})^{+}, 85\right]$; HRMS $(\mathrm{M}+\mathrm{Na})^{+}$calcd for $\mathrm{C}_{23} \mathrm{H}_{26} \mathrm{O}_{5}$ 405.1678, found 405.1682.

(1S,3aS,4S,7R,7aR)-2,2,7-Trimethyl-9-oxo-2,3,3a,4,7,7a-hexahydro-1H-4,7-ethan

-oinden-1-yl Acetate (49). A magnetically stirred solution of compound 48 (3.30 g, $8.63 \mathrm{mmol}$ ) in deoxygenated THF/methanol (30 $\mathrm{mL}$ of a 9:1 v/v mixture) maintained at $-78{ }^{\circ} \mathrm{C}$ under an argon atmosphere was treated, dropwise over $1 \mathrm{~h}$, with samarium(II) iodide (ca. $250 \mathrm{~mL}$ of a $0.086 \mathrm{M}$ solution in THF) at which point a dark-blue color persisted for $0.25 \mathrm{~h}$. The resulting mixture was then quenched with $\mathrm{NH}_{4} \mathrm{Cl}$ (100 mL of a saturated aqueous solution) and the ensuing suspension extracted with diethyl ether $(2 \times 100 \mathrm{~mL})$. The combined organic phases were washed with $\mathrm{HCl}$ $(2 \times 50 \mathrm{~mL}$ of a $1 \mathrm{M}$ aqueous solution) and brine $(1 \times 100 \mathrm{~mL})$ before being dried ( $\left.\mathrm{Na}_{2} \mathrm{SO}_{4}\right)$, filtered and concentrated under reduced pressure. The residue thus obtained was subjected to flash column chromatography (silica, 1:9 v/v diethyl ether/n-hexane elution) and gave, after concentration of the appropriate fractions $\left[R_{\mathrm{f}}=0.4(5)\right.$ in $1: 9$ v/v diethyl ether/n-hexane)], compound $49(1.91 \mathrm{~g}, 85 \%)$ as a clear, colorless oil, $[\alpha]_{\mathrm{D}}$ 
$=+161.0\left({ }^{c} 1.0, \mathrm{CHCl}_{3}\right) .{ }^{1} \mathrm{H} \mathrm{NMR}\left(400 \mathrm{MHz}, \mathrm{CDCl}_{3}\right) \delta 6.10(\mathrm{~d}, J=8.1 \mathrm{~Hz}, 1 \mathrm{H}), 5.86$ (t, $J=8.1 \mathrm{~Hz}, 1 \mathrm{H}), 4.93$ (d, $J=6.0 \mathrm{~Hz}, 1 \mathrm{H}), 2.96$ (dd, $J=6.0$ and $1.8 \mathrm{~Hz}, 1 \mathrm{H}$ ), 2.60 (m, 1H), 2.46 (m, 1H), 1.98 (s, 3H), 1.79 (s, 2H), 1.38 (m, 1H), 1.26 (t, J = $11.5 \mathrm{~Hz}$, 1H), 1.05 (s, 3H), 0.90 (s, 3H), 0.76 (s, 3H); ${ }^{13} \mathrm{C}$ NMR (100 MHz, $\left.\mathrm{CDCl}_{3}\right) \delta$ 212.3, 170.0, 142.9, 123.0, 80.4, 53.6, 52.1, 47.7, 44.8, 42.0, 41.2, 39.2, 25.4, 22.2, 22.0, 21.4; IR $v_{\max }$ 2965, 1726, 1373, 1239, 1069, $707 \mathrm{~cm}^{-1}$; MS (ESI, +ve) $\mathrm{m} / \mathrm{z} 547$ $\left[(2 \mathrm{M}+\mathrm{Na})^{+}, 10 \%\right], 285\left[(\mathrm{M}+\mathrm{Na})^{+}, 100\right], 263\left[(\mathrm{M}+\mathrm{H})^{+}, 1\right]$; HRMS (ESI, +ve) $(\mathrm{M}+\mathrm{H})^{+}$ calcd for $\mathrm{C}_{16} \mathrm{H}_{22} \mathrm{O}_{3} 263.1647$, found 263.1649.

$(1 R, 2 \mathrm{a} R, 4 \mathrm{a} R, 7 S, 7 \mathrm{a} R, 7 \mathrm{~b} S)-1-(M e t h o x y m e t h o x y)-6,6,7 \mathrm{~b}-t r i m e t h y l-2-0 x o-2,2 \mathrm{a}, 4 \mathrm{a}, 5$, -6,7,7a,7b-octahydro-1H-cyclobuta[e]inden-7-yl Acetate (50). A magnetically stirred solution of compound 45 (100 $\mathrm{mg}, 0.31 \mathrm{mmol})$ in dry, deoxygenated dichloromethane (30 mL) maintained under a nitrogen atmosphere was irradiated with a Hanovia 450W medium pressure mercury-vapor lamp for $16 \mathrm{~h}$. The reaction mixture was then cooled and concentrated under reduced pressure and the residue thus obtained subjected to flash column chromatography (silica, 1:19 v/v ethyl acetate/n-hexane elution) to afford two fractions, $\mathrm{A}$ and B.

Concentration of fraction A $\left[R_{\mathrm{f}}=0.3(5)\right.$ in $1: 19 \mathrm{v} / \mathrm{v}$ ethyl acetate $/ n$-hexane] gave compound 50 (43 mg, $43 \%$ or $99 \%$ brsm) as a clear, colorless oil, $[\alpha]_{D}=-157.0$ (c 0.7, $\left.\mathrm{CHCl}_{3}\right) .{ }^{1} \mathrm{H}$ NMR (400 MHz, $\left.\mathrm{CDCl}_{3}\right) \delta 5.76(\mathrm{~m}, 1 \mathrm{H}), 5.73(\mathrm{~m}, 1 \mathrm{H}), 5.54(\mathrm{~m}$, 1H), 4.97 (d, $J=4.2 \mathrm{~Hz}, 1 \mathrm{H}), 3.02(\mathrm{~m}, 1 \mathrm{H}), 2.96(\mathrm{~m}, 1 \mathrm{H}), 2.87$ (dd, $J=9.3$ and 4.2 Hz, 1H), 2.14 (s, 3H), 2.06 (m, 1H), 1.98 (s, 3H), 1.51 (dd, $J=13.3$ and $5.0 \mathrm{~Hz}, 1 \mathrm{H}$ ), 1.09 (s, 3H), 1.03 (s, 3H), 0.92 (s, 3H); ${ }^{13} \mathrm{C}$ NMR (100 MHz, CDCl $) \delta 201.3,170.6$, 169.9, 134.3, 117.2, 83.4, 83.2, 59.0, 45.4, 43.5, 42.8, 34.8, 34.7, 28.8, 24.3, 21.3, 20.5, 18.4; IR $v_{\max }$ 2933, 2873, 1792, 1735, 1373, 1224, $1087 \mathrm{~cm}^{-1}$; MS (ESI, +ve) 
m/z $375\left[(\mathrm{M}+\mathrm{MeOH}+\mathrm{Na})^{+}, 20 \%\right], 343\left[(\mathrm{M}+\mathrm{Na})^{+}, 100\right] ; \mathrm{HRMS}(\mathrm{M}+\mathrm{Na})^{+}$calcd for $\mathrm{C}_{18} \mathrm{H}_{24} \mathrm{O}_{5} 343.1521$, found 343.1520.

Concentration of the fraction $\mathrm{B}\left[R_{\mathrm{f}}=0.2(5)\right.$ in $1: 19 \mathrm{v} / \mathrm{v}$ ethyl acetate/n-hexane] gave compound 45 (56 mg, 56\% recovery) as a colorless, crystalline solid that was identical, in all respects, with an authentic sample.

$(1 R, 2 \mathrm{a} R, 4 \mathrm{a} R, 7 S, 7 \mathrm{a} R, 7 \mathrm{bS})-1-(M e t h o x y m e t h o x y)-6,6,7 \mathrm{~b}-t r i m e t h y l-2-0 x 0-2,2 \mathrm{a}, 4 \mathrm{a}, 5$, -6,7,7a,7b-octahydro-1H-cyclobuta[e]inden-7-yl Acetate (51). A magnetically stirred solution of compound 46 (100 mg, $0.31 \mathrm{mmol})$ in dry, deoxygenated dichloromethane $(30 \mathrm{~mL})$ maintained under a nitrogen atmosphere was irradiated with a Hanovia 450W medium pressure mercury-vapor lamp for $16 \mathrm{~h}$. The cooled reaction mixture was concentrated under reduced pressure and the residue thus obtained subjected to flash column chromatography (silica, 1:9 v/v ethyl acetate/n-hexane elution) and thus affording two fractions, A and B.

Concentration of the fraction A $\left[R_{\mathrm{f}}=0.3(5)\right.$ in $1: 9 \mathrm{v} / \mathrm{v}$ ethyl acetate/n-hexane] gave compound 51 (27 mg, $27 \%$ or $98 \%$ brsm) as a clear, colorless oil, $[\alpha]_{D}=-324.0$ (c 0.2, $\left.\mathrm{CHCl}_{3}\right) .{ }^{1} \mathrm{H}$ NMR (400 MHz, $\left.\mathrm{CDCl}_{3}\right) \delta 5.70(\mathrm{~m}, 1 \mathrm{H}), 5.55$ (m, 1H), 5.00 (d, $J$ $=4.1 \mathrm{~Hz}, 1 \mathrm{H}), 4.67(\mathrm{~m}, 3 \mathrm{H}), 3.42(\mathrm{~s}, 3 \mathrm{H}), 2.90(\mathrm{~m}, 1 \mathrm{H}), 2.85(\mathrm{~m}, 1 \mathrm{H}), 2.76(\mathrm{dd}, J=$ 9.4 and $4.1 \mathrm{~Hz}, 1 \mathrm{H}), 2.05$ (m, 1H), 1.98 (s, 3H), 1.51 (dd, $J=13.2$ and $5.2 \mathrm{~Hz}, 1 \mathrm{H}$ ), 1.11 (s, 3H), 1.06 (s, 3H), 0.93 (s, 3H); $\left.{ }^{13} \mathrm{C} \mathrm{NMR} \mathrm{(100} \mathrm{MHz,} \mathrm{CDCl}_{3}\right) \delta 204.5,170.7$, 133.4, 118.0, 97.3, 89.4, 83.2, 58.5, 56.1, 45.4, 43.5, 43.2, 34.7, 34.4, 28.7, 24.2, 21.4, 18.5; IR $v_{\max } 2962,2933,1787,1733,1373,1240,1110,1067,1012 \mathrm{~cm}^{-1}$; MS (ESI, +ve) $m / z 345\left[(\mathrm{M}+\mathrm{Na})^{+}, 100 \%\right]$; HRMS $(\mathrm{M}+\mathrm{Na})^{+}$calcd for $\mathrm{C}_{18} \mathrm{H}_{26} \mathrm{O}_{5} 345.1678$, found 345.1672. 
Concentration of the fraction B $\left[R_{\mathrm{f}}=0.2(5)\right.$ in $1: 9 \mathrm{v} / \mathrm{v}$ ethyl acetate $/ n$-hexane] gave compound 46 (71 mg, 71\% recovery) as a clear, colorless oil that was identical, in all respects, with an authentic sample.

(3S,3aR,4R,7S,7aS,9R)-9-((tert-Butyldimethylsilyl)oxy)-2,2,4-trimethyl-8-oxo-2,3, -3a,4,7,7a-hexahydro-1H-4,7-ethanoinden-3-yl Acetate (52). A magnetically stirred solution of compound 47 (100 mg, $0.25 \mathrm{mmol}$ ) in dry, deoxygenated dichloromethane (30 mL) maintained under a nitrogen atmosphere was irradiated with a Hanovia 450W medium pressure mercury-vapor lamp for $16 \mathrm{~h}$. The cooled reaction mixture was concentrated under reduced pressure and the residue thus obtained subjected to flash column chromatography (silica, 1:19 v/v ethyl acetate/n-hexane elution) and thereby affording two fractions, A and B.

Concentration of the fraction A $\left(R_{\mathrm{f}}=0.4\right.$ in $1: 19 \mathrm{v} / \mathrm{v}$ ethyl acetate $/ n$-hexane $)$ gave compound 52 (18 mg, $18 \%$ or $99 \%$ brsm) as a clear, colorless oil, $[\alpha]_{\mathrm{D}}=-189.0$ (c 2.3, $\left.\mathrm{CHCl}_{3}\right) .{ }^{1} \mathrm{H}$ NMR (400 MHz, $\left.\mathrm{CDCl}_{3}\right) \delta 5.64$ (m, 1H), 5.55 (m, 1H), 4.98 (d, J $=4.1 \mathrm{~Hz}, 1 \mathrm{H}), 4.71(\mathrm{~d}, J=2.8 \mathrm{~Hz}, 1 \mathrm{H}), 2.86-2.78$ (complex m, 2H), 2.65 (dd, $J=9.3$ and $4.1 \mathrm{~Hz}, 1 \mathrm{H}), 2.03$ (m, 1H), 1.97 (s, 3H), 1.50 (dd, $J=13.2$ and $5.1 \mathrm{~Hz}, 1 \mathrm{H}), 1.10$ (s, 3H), 0.97 (s, 3H), 0.92 (s, 3H), 0.90 (s, 9H), 0.10 (s, 3H), 0.07 (s, 3H); ${ }^{13} \mathrm{C}$ NMR $\left(100 \mathrm{MHz}, \mathrm{CDCl}_{3}\right) \delta 206.1,170.6,132.8,118.7,85.0,83.3,58.0,45.5,43.5,43.2$, $34.8,34.5,28.8,25.8,24.2,21.3,18.4,18.2,-4.6,-4.9$; IR $v_{\max } 2958,2931,2859$, 1788, 1736, 1239, 1108, 883, 838, $780 \mathrm{~cm}^{-1}$; MS (ESI, +ve) $\mathrm{m} / \mathrm{z} 415\left[(\mathrm{M}+\mathrm{Na})^{+}\right.$, 100\%], $393\left[(\mathrm{M}+\mathrm{H})^{+}, 5\right]$; HRMS $(\mathrm{M}+\mathrm{Na})^{+}$calcd for $\mathrm{C}_{22} \mathrm{H}_{36} \mathrm{O}_{4} \mathrm{Si} 415.2281$, found 415.2284.

Concentration of the fraction B $\left(R_{\mathrm{f}}=0.3\right.$ in $1: 19 \mathrm{v} / \mathrm{v}$ ethyl acetate/ $n$-hexane) gave compound 47 (81 mg, 81\% recovery) as a colorless, crystalline solid that was identical, in all respects, with an authentic sample. 
$(1 R, 2 \mathrm{a} R, 4 \mathrm{a} R, 7 S, 7 \mathrm{a} R, 7 \mathrm{~b} S)-7-A c e t o x y-6,6,7 \mathrm{~b}-t$ rimethyl-2-oxo-2,2a,4a,5,6,7,7a,7b-

octahydro-1H-cyclobuta[e]inden-1-yl Benzoate (53). A magnetically stirred solution of compound 48 (100 mg, $0.26 \mathrm{mmol}$ ) in dry, deoxygenated dichloromethane (30 mL) maintained under a nitrogen atmosphere was irradiated with a Hanovia 450W medium pressure mercury-vapor lamp for $16 \mathrm{~h}$. The reaction mixture was then cooled and concentrated under reduced pressure and the residue so obtained subjected to flash column chromatography (silica, 1:19 v/v ethyl acetate/n-hexane elution) and thereby affording two fractions, A and B.

Concentration of the fraction $\mathrm{A}\left[R_{\mathrm{f}}=0.3(5)\right.$ in $1: 19 \mathrm{v} / \mathrm{v}$ ethyl acetate $/ n$-hexane] gave compound 53 (17 mg, 17\% or $100 \%$ brsm) as a colorless, crystalline solid, $\mathrm{mp}=$ $180-181{ }^{\circ} \mathrm{C},[\alpha]_{\mathrm{D}}=-672.0\left(c\right.$ 1.5, $\left.\mathrm{CHCl}_{3}\right) .{ }^{1} \mathrm{H}$ NMR $\left(400 \mathrm{MHz}, \mathrm{CDCl}_{3}\right) \delta 8.06(\mathrm{~m}$, 2H), 7.59 (t, $J=7.4 \mathrm{~Hz}, 1 \mathrm{H}), 7.45$ (t, $J=7.4 \mathrm{~Hz}, 2 \mathrm{H}), 5.97$ (d, $J=2.8 \mathrm{~Hz}, 1 \mathrm{H}), 5.80$ (m, 1H), 5.59 (m, 1H), 4.98 (d, J = $4.2 \mathrm{~Hz}, 1 \mathrm{H}), 3.10$ (m, 1H), 3.04 (m, 1H), 2.97 (dd, $J=9.3$ and $4.2 \mathrm{~Hz}, 1 \mathrm{H}), 2.08$ (dd, $J=13.3$ and $9.8 \mathrm{~Hz}, 1 \mathrm{H}), 1.99$ (s, 3H), 1.53 (dd, $J=$ 13.3 and $5.0 \mathrm{~Hz}, 1 \mathrm{H}), 1.12$ (s, 3H), 1.10 (s, 3H), 0.92 (s, 3H); ${ }^{13} \mathrm{C}$ NMR (100 MHz, $\left.\mathrm{CDCl}_{3}\right) \delta 201.1,170.6,165.3,134.3,133.8,130.1,128.8,128.7,117.3,83.6,83.2$, 59.2, 45.4, 43.5, 42.9, 35.1, 34.8, 28.8, 24.3, 21.3, 18.5; IR $v_{\max } 2963$, 2934, 2872, 1790, 1724, 1372, 1237, 1104, $709 \mathrm{~cm}^{-1}$; MS (ESI, +ve) m/z 787 [(2M+Na), $\left.10 \%\right]$, $437\left[(\mathrm{M}+\mathrm{MeOH}+\mathrm{Na})^{+}, 50\right], 405\left[(\mathrm{M}+\mathrm{Na})^{+}, 100\right] ; \mathrm{HRMS}(\mathrm{M}+\mathrm{Na})^{+}$calcd for $\mathrm{C}_{23} \mathrm{H}_{26} \mathrm{O}_{5}$ 405.1678, found 405.1670.

Concentration of the fraction $\mathrm{B}\left[R_{\mathrm{f}}=0.2(5)\right.$ in $1: 19 \mathrm{v} / \mathrm{v}$ ethyl acetate $/ n$-hexane $]$ gave compound $\mathbf{4 8}$ (83 $\mathrm{mg}, 83 \%$ recovery) as a colorless, crystalline solid that was identical, in all respects, with an authentic sample.

(2aR,4aR,7S,7aR,7bS)-6,6,7b-Trimethyl-2-oxo-2,2a,4a,5,6,7,7a,7b-octahydro-1Hcyclobuta[e]inden-7-yl Acetate (54). A magnetically stirred solution of compound 
49 (100 mg, $0.38 \mathrm{mmol})$ in dry, deoxygenated dichloromethane (30 mL) maintained under a nitrogen atmosphere was irradiated with a Hanovia 450W medium pressure mercury-vapor lamp for $16 \mathrm{~h}$. The reaction mixture was then cooled and concentrated under reduced pressure and the ensuing residue subjected to flash column chromatography (silica, 1:19 v/v ethyl acetate/n-hexane elution) and thus affording two fractions, A and B.

Concentration of the fraction $\mathrm{A}\left[R_{\mathrm{f}}=0.3(5)\right.$ in $1: 19 \mathrm{v} / \mathrm{v}$ ethyl acetate $/ n$-hexane] gave compound 54 (23 mg, 23\% or $92 \%$ brsm) as a clear, colorless oil, $[\alpha]_{\mathrm{D}}=-254.0$ (c 3.4, $\left.\mathrm{CHCl}_{3}\right) .{ }^{1} \mathrm{H}$ NMR (400 MHz, $\left.\mathrm{CDCl}_{3}\right) \delta 5.73(\mathrm{~m}, 1 \mathrm{H}), 5.48(\mathrm{~m}, 1 \mathrm{H}), 4.97$ (d, $J$ $=4.2 \mathrm{~Hz}, 1 \mathrm{H}$ ), 3.12 (dd, $J=16.2$ and $2.1 \mathrm{~Hz}, 1 \mathrm{H}), 3.05$ (m, 1H), 2.84 (m, 1H), 2.59 (dd, $J=9.2$ and $4.3 \mathrm{~Hz}, 1 \mathrm{H}$ ), 2.51 (dd, $J=16.2$ and $6.0 \mathrm{~Hz}, 1 \mathrm{H}$ ), 2.01 (dd, $J=13.2$ and $9.7 \mathrm{~Hz}, 1 \mathrm{H}), 1.95$ (s, 3H), 1.50 (dd, $J=13.2$ and $4.8 \mathrm{~Hz}, 1 \mathrm{H}), 1.19$ (s, 3H), 1.08 (s, 3H), 0.90 (s, 3H); ${ }^{13} \mathrm{C}$ NMR (100 MHz, $\left.\mathrm{CDCl}_{3}\right) \delta$ 206.4, 170.7, 133.7, 118.2, 83.5, 64.1, 56.7, 45.7, 44.3, 43.4, 34.9, 29.0, 28.1, 25.5, 24.3, 21.3; IR $v_{\max } 3020,2966$, 2871, 1778, 1731, 1373, 1239, 1020, $711 \mathrm{~cm}^{-1}$; MS (ESI, +ve) m/z $285\left[(\mathrm{M}+\mathrm{Na})^{+}\right.$, 100\%]; HRMS (M+Na) ${ }^{+}$calcd for $\mathrm{C}_{16} \mathrm{H}_{22} \mathrm{O}_{3}$ 285.1467, found 285.1472.

Concentration of the fraction B $\left(R_{\mathrm{f}}=0.3\right.$ in $1: 19 \mathrm{v} / \mathrm{v}$ ethyl acetate $/ n$-hexane $)$ gave compound 49 (69 mg, 69\% recovery) as a colorless, crystalline solid that was identical, in all respects, with an authentic sample.

$(1 S, 1 \mathrm{a} R, 3 \mathrm{a} R, 6 S, 6 \mathrm{a} R, 6 \mathrm{~b} R)-5,5,6 \mathrm{~b}-\mathrm{Trimethyl}-1,1 \mathrm{a}, 3 \mathrm{a}, 4,5,6,6 \mathrm{a}, 6 \mathrm{~b}-0 \mathrm{ctahydrocyclopr}$ -opa[e]indene-1,6-diyl Diacetate (55). A magnetically stirred solution of compound 50 (40 mg, $0.12 \mathrm{mmol})$ in dry, deoxygenated dichloromethane $(10 \mathrm{~mL})$ maintained under a nitrogen atmosphere was irradiated with a Hanovia 450W medium pressure mercury-vapor lamp for $0.5 \mathrm{~h}$. The reaction mixture was then cooled and concentrated under reduced pressure and the residue thus obtained subjected to flash column 
chromatography (silica, 1:9 v/v ethyl acetate/n-hexane elution). Concentration of the appropriate fractions $\left[R_{\mathrm{f}}=0.6(5)\right.$ in $1: 9 \mathrm{v} / \mathrm{v}$ ethyl acetate/ $n$-hexane $]$ gave compound 55 (39 mg, 98\%) as a clear, colorless oil, $[\alpha]_{\mathrm{D}}=+167.0$ (c 0.5, $\left.\mathrm{CHCl}_{3}\right) .{ }^{1} \mathrm{H}$ NMR (400 $\left.\mathrm{MHz}, \mathrm{CDCl}_{3}\right) \delta 5.90(\mathrm{~m}, 1 \mathrm{H}), 5.22$ (dd, $J=10.0$ and $\left.1.9 \mathrm{~Hz}, 1 \mathrm{H}\right), 5.04$ (d, $J=3.9 \mathrm{~Hz}$, 1H), 3.77 (d, $J=2.3 \mathrm{~Hz}, 1 \mathrm{H}), 2.82$ (dd, $J=10.1$ and $3.9 \mathrm{~Hz}, 1 \mathrm{H}), 2.64$ (m, 1H), 2.05 (s, 3H), 2.00 (s, 3H), 1.90 (dd, $J=13.1$ and $9.6 \mathrm{~Hz}, 1 \mathrm{H}$ ), 1.33 (dd, $J=13.1$ and 5.2 Hz, 1H), 1.22 (dd, $J=11.1$ and $2.3 \mathrm{~Hz}, 1 \mathrm{H}), 1.06$ (s, 6H), 0.90 (s, 3H); ${ }^{13} \mathrm{C}$ NMR (100 $\left.\mathrm{MHz}, \mathrm{CDCl}_{3}\right) \delta 171.8,171.1,129.9,123.1,84.0,63.4,46.6,42.8,40.8,35.4,28.6$, 24.3, 24.2, 22.1, 21.3, 20.9, 15.9; IR $v_{\max }$ 3026, 2966, 2933, 2872, 1733, 1371, 1227 $\mathrm{cm}^{-1}$; MS (ESI, +ve) m/z 347 [(M+MeOH+Na) $\left.{ }^{+}, 100 \%\right], 331$ [(M+K) $\left.)^{+}, 20\right], 329$ (50), $315\left[(\mathrm{M}+\mathrm{Na})^{+}\right.$, 60]; HRMS $(\mathrm{M}+\mathrm{Na})^{+}$calcd for $\mathrm{C}_{17} \mathrm{H}_{24} \mathrm{O}_{4}$ 315.1572, found 315.1570.

$(1 S, 1 \mathrm{a} R, 3 \mathrm{a} R, 6 S, 6 \mathrm{a} R, 6 \mathrm{~b} S)-1-((t e r t-B u t y l d i m e t h y l s i l y l) 0 x y)-5,5,6 \mathrm{~b}-$ trimethyl-1,1a,3a,4,5,6,6a,6b-octahydrocyclopropa[e]inden-6-yl Acetate (56). A magnetically stirred solution of compound 47 (100 mg, $0.25 \mathrm{mmol})$ in dry, deoxygenated dichloromethane (30 mL) maintained under a nitrogen atmosphere was irradiated with a Hanovia 450W medium pressure mercury-vapor lamp for $1.67 \mathrm{~h}$. The reaction mixture was then cooled, concentrated under reduced pressure and the residue thus obtained subjected to flash column chromatography (silica, 1:19 v/v ethyl acetate/4060 petroleum ether) to afford two fractions, A and B.

Concentration of the fraction A $\left(R_{\mathrm{f}}=0.6\right.$ in $1: 4 \mathrm{v} / \mathrm{v}$ ethyl acetate/40-60 petroleum ether) gave compound 56 (74 $\mathrm{mg}, 80 \%$ or $87 \%$ brsm) as a clear, colorless oil, $[\alpha]_{\mathrm{D}}=-65.5\left(\right.$ c 1.0, $\left.\mathrm{CHCl}_{3}\right) .{ }^{1} \mathrm{H}$ NMR $\left[400 \mathrm{MHz},\left(\mathrm{CD}_{3}\right)_{2} \mathrm{CO}\right] \delta 5.92(\mathrm{~m}, 1 \mathrm{H}), 5.11$ (dd, $J=10.0$ and $2.1 \mathrm{~Hz}, 1 \mathrm{H}$ ), 5.01 (d, $J=4.1 \mathrm{~Hz}, 1 \mathrm{H}$ ), 3.14 (d, $J=2.2 \mathrm{~Hz}, 1 \mathrm{H}$ ), 2.78 (m, 1H), 2.63 (m, 1H), 1.95 (s, 3H), 1.89 (m, 1H), 1.30 (dd, $J=13.0$ and $5.2 \mathrm{~Hz}, 1 \mathrm{H})$, 1.09 (s, 3H), 1.06 (s, 3H), 0.97 (dd, $J=5.5$ and $2.4 \mathrm{~Hz}, 1 \mathrm{H}), 0.90$ (s, 9H), 0.87 (s, 
3H), 0.10(1) (s, 3H), 0.09(8) (s, 3H); ${ }^{13} \mathrm{C}$ NMR [100 MHz, $\left.\left(\mathrm{CD}_{3}\right)_{2} \mathrm{CO}\right] \delta$ 170.7, 128.9, 125.2, 84.4, 63.9, 47.3, 43.3, 41.9, 36.2, 28.9, 27.7, 26.2, 24.7, 23.1, 21.1, 18.6, 15.9, -4.9 (one signal obscured or overlapping); IR $v_{\max } 3021,2958,2930,2859,1734$, 1240, 1153, 859, 835, $776 \mathrm{~cm}^{-1}$; MS (ESI, +ve) m/z 387 [(M+Na) $\left.)^{+}, 100 \%\right]$; HRMS $(\mathrm{M}+\mathrm{Na})^{+}$calcd for $\mathrm{C}_{21} \mathrm{H}_{36} \mathrm{O}_{3} \mathrm{Si} 387.2331$, found 387.2331.

Concentration of the fraction B $\left(R_{\mathrm{f}}=0.5\right.$ in $1: 4 \mathrm{v} / \mathrm{v}$ ethyl acetate $/ 40-60$ petroleum ether) gave compound 47 (8 $\mathrm{mg}$, 8\% recovery) as a white, crystalline solid that was identical, in all respects, with an authentic sample.

(3S,3aR,4R,7S,7aS,9R)-2,2,4-Trimethyl-9-((methylsulfonyl)oxy)-8-oxo-2,3,3a,4,7,7a-hexahydro-1H-4,7-ethanoinden-3-yl Acetate (57). A magnetically stirred solution of compound 44 (280 mg, $1.01 \mathrm{mmol}$ ), methanesulfonyl chloride (94 $1.21 \mathrm{mmol})$ and triethylamine (212

maintained under a nitrogen atmosphere was stirred at $22{ }^{\circ} \mathrm{C}$ for $48 \mathrm{~h}$ then quenched with $\mathrm{NH}_{4} \mathrm{Cl}$ (10 $\mathrm{mL}$ of a saturated aqueous solution) before being extracted with dichloromethane $(2 \times 50 \mathrm{~mL})$. The combined organic fractions were washed with water $(2 \times 50 \mathrm{~mL})$ and brine $(1 \times 50 \mathrm{~mL})$ then dried $\left(\mathrm{MgSO}_{4}\right)$, filtered, and concentrated under reduced pressure. The residue thus obtained was subjected to flash column chromatography (silica, 1:9 v/v ethyl acetate/40-60 petroleum ether elution) to give, after concentration of the appropriate fractions $\left(R_{\mathrm{f}}=0.3\right.$ in $1: 9 \mathrm{v} / \mathrm{v}$ ethyl acetate/40-60 petroleum ether), compound 57 (198 mg, 56\%) as a light-yellow oil, $[\alpha]_{\mathrm{D}}=+114.0\left(c\right.$ 4.3, $\left.\mathrm{CHCl}_{3}\right) .{ }^{1} \mathrm{H} \mathrm{NMR}\left(400 \mathrm{MHz}, \mathrm{CDCl}_{3}\right) \delta 6.08(\mathrm{~d}, J=8.1 \mathrm{~Hz}, 1 \mathrm{H})$, 5.95 (t, $J=8.1 \mathrm{~Hz}, 1 \mathrm{H}), 5.08$ (d, $J=5.9 \mathrm{~Hz}, 1 \mathrm{H}), 4.31$ (s, 1H), 3.19 (m, 1H), 3.17 (s, 3H), 2.69 (m, 1H), 2.58 (dd, $J=10.6$ and $6.0 \mathrm{~Hz}, 1 \mathrm{H}$ ), 2.05 (s, 3H), 1.53 (m, 1H), 1.39 (t, $J=12.0 \mathrm{~Hz}, 1 \mathrm{H}), 1.20$ (s, 3H), 0.98 (s, 3H), 0.85 (s, 3H); ${ }^{13} \mathrm{C}$ NMR (100 $\left.\mathrm{MHz}, \mathrm{CDCl}_{3}\right) \delta 204.1,170.0,140.9,121.9,81.3,81.0,50.7,50.2,44.9,42.7,41.6$, 
39.5(1), 39.4(8), 25.3, 22.1, 21.5, 18.1; IR $v_{\max } 2968,1730,1358,1238,1173,965$, $841 \mathrm{~cm}^{-1}$; MS (ESI, +ve) m/z $735\left[(2 \mathrm{M}+\mathrm{Na})^{+}, 15 \%\right], 379\left[(\mathrm{M}+\mathrm{Na})^{+}, 100\right]$; HRMS $(\mathrm{M}+\mathrm{Na})^{+}$calcd for $\mathrm{C}_{17} \mathrm{H}_{24} \mathrm{O}_{6} \mathrm{~S} 379.1191$, found 379.1193.

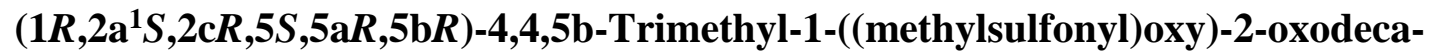
hydro-1H-cyclopenta[a]cyclopropa[cd]pentalen-5-yl Acetate (58). A magnetically stirred solution of compound 57 (100 mg, $0.28 \mathrm{mmol})$ in dry, deoxygenated dichloromethane (30 mL) maintained under a nitrogen atmosphere was irradiated with a Hanovia 450W medium pressure mercury-vapor lamp for $12 \mathrm{~h}$. The reaction mixture was then cooled and concentrated under reduced pressure and the residue thus obtained subjected to flash column chromatography (silica, 1:9 v/v ethyl acetate/n-hexane elution) to afford two fractions, $\mathrm{A}$ and $\mathrm{B}$.

Concentration of the fraction A $\left[R_{\mathrm{f}}=0.4(5)\right.$ in $1: 9 \mathrm{v} / \mathrm{v}$ ethyl acetate/n-hexane] gave compound 58 (48 mg, $48 \%$ or $98 \%$ brsm) as a clear, colorless oil, $[\alpha]_{\mathrm{D}}=+161.0$ (c 1.0, $\left.\mathrm{CHCl}_{3}\right) .{ }^{1} \mathrm{H} \mathrm{NMR}\left(400 \mathrm{MHz}, \mathrm{CDCl}_{3}\right) \delta 5.54$ (s, $\left.1 \mathrm{H}\right), 5.09$ (d, $\left.J=4.8 \mathrm{~Hz}, 1 \mathrm{H}\right)$, 3.05 (s, 3H), 2.85-2.72 (complex m, 2H), 2.50 (d, $J=4.8 \mathrm{~Hz}, 1 \mathrm{H}$ ), 2.23 (s, 3H), 2.18 (s, 1H), 1.71 (d, $J=4.8 \mathrm{~Hz}, 1 \mathrm{H}), 1.59$ (dd, $J=8.7$ and $2.3 \mathrm{~Hz}, 2 \mathrm{H}), 1.07$ (s, 3H), 0.97(2) (s, 3H), 0.96(7) (s, 3H); ${ }^{13} \mathrm{C}$ NMR (100 MHz, $\left.\mathrm{CDCl}_{3}\right) \delta$ 211.1, 170.2, 80.9, 78.8, 50.4, 45.2, 42.5, 42.4, 41.1, 39.3, 37.6, 36.1, 32.9, 25.1, 22.7, 22.5, 21.2; IR $v_{\max }$ 2966, 2936, 2875, 1729, 1356, 1236, 1172, 917, $842 \mathrm{~cm}^{-1}$; MS m/z $735\left[(2 \mathrm{M}+\mathrm{Na})^{+}\right.$, 5\%], $379\left[(\mathrm{M}+\mathrm{Na})^{+}, 100\right]$; HRMS $(\mathrm{M}+\mathrm{Na})^{+}$calcd for $\mathrm{C}_{17} \mathrm{H}_{24} \mathrm{O}_{6} \mathrm{~S} 379.1191$, found 379.1189.

Concentration of the fraction $\mathrm{B}\left(R_{\mathrm{f}}=0.3\right.$ in $1: 9 \mathrm{v} / \mathrm{v}$ ethyl acetate $/ n$-hexane) gave compound 57 (50 mg, 50\% recovery) as a clear, colorless oil that was identical, in all respects, with an authentic sample.

\section{X-ray Crystallographic Studies}




\section{Crystallographic Data.}

Compound 8. $\mathrm{C}_{9} \mathrm{H}_{12} \mathrm{O}_{3}, M=168.19, T=150 \mathrm{~K}$, orthorhombic, space group $P 2{ }_{1} 2_{1} 2_{1}, Z$ $=4, a=7.3238(1) \AA, b=7.5553(1) \AA, c=14.5389(2) \AA ; V=804.49(2) \AA^{3}, D_{x}=$ $1.389 \mathrm{~g} \mathrm{~cm}^{-3}, 1585$ unique data $\left(2 \theta_{\max }=144.6^{\circ}\right), R=0.029$ [for 1569 reflections with $I>2.0 \sigma(I)] ; R w=0.057$ (all data), $S=1.00$.

Compound 9. $\mathrm{C}_{15} \mathrm{H}_{26} \mathrm{O}_{3} \mathrm{Si}, M=282.46, T=150 \mathrm{~K}$, monoclinic, space group $C 2, Z=$ 4, $a=18.9377(5) \AA, b=6.2442(1) \AA, c=14.6275(5) \AA ; \beta=105.950(3)^{\circ} ; V=$ 1663.12(8) $\AA^{3}, D_{x}=1.128 \mathrm{~g} \mathrm{~cm}^{-3}, 3042$ unique data $\left(2 \theta_{\max }=144.8^{\circ}\right), R=0.044$ [for 2889 reflections with $I>2.0 \sigma(I)]$; $R w=0.080$ (all data), $S=0.99$.

Compound 10. $\mathrm{C}_{9} \mathrm{H}_{12} \mathrm{O}_{3}, M=168.19, T=150 \mathrm{~K}$, orthorhombic, space group $P 2{ }_{1} 2_{1}{ }_{1}$, $Z=8, a=8.23350(7) \AA, b=8.68949(8) \AA, c=23.6396(2) \AA ; V=1691.29(3) \AA^{3}, D_{x}$ $=1.321 \mathrm{~g} \mathrm{~cm}^{-3}, 3334$ unique data $\left(2 \theta_{\max }=144.6^{\circ}\right), R=0.051$ [for 3257 reflections with $I>2.0 \sigma(I)] ; R w=0.112$ (all data), $S=1.01$.

Compound 13. $\mathrm{C}_{14} \mathrm{H}_{26} \mathrm{O}_{2} \mathrm{Si}, M=254.45, T=150 \mathrm{~K}$, orthorhombic, space group $P 2{ }_{1}{ }_{1} 2_{1}, Z=8, a=18.3637(1) \AA, b=14.1093(1) \AA, c=12.0122(1) \AA ; V=$ 3112.35(4) $\AA^{3}, D_{x}=1.086 \mathrm{~g} \mathrm{~cm}^{-3}, 6159$ unique data $\left(2 \theta_{\max }=145.0^{\circ}\right), R=0.029$ [for 6101 reflections with $I>2.0 \sigma(I)] ; R w=0.067$ (all data), $S=1.01$.

Compound 23. $\mathrm{C}_{13} \mathrm{H}_{16} \mathrm{O}_{3}, M=220.27, T=150 \mathrm{~K}$, orthorhombic, space group $P 2{ }_{1} 2_{1} 2_{1}$, $Z=4, a=7.27015(5) \AA, b=11.05109(8) \AA, c=13.78627(9) \AA ; V=1107.63(1) \AA^{3}$, $D_{x}=1.321 \mathrm{~g} \mathrm{~cm}^{-3}, 2186$ unique data $\left(2 \theta_{\max }=144.8^{\circ}\right), R=0.019$ [for 2152 reflections with $I>2.0 \sigma(I)] ; R w=0.057$ (all data), $S=1.01$.

Compound 27. $\mathrm{C}_{13} \mathrm{H}_{16} \mathrm{O}_{3}, M=220.27, T=200 \mathrm{~K}$, monoclinic, space group $P 2{ }_{1}, Z=2$, $a=7.2003(2) \AA, b=8.4682(2) \AA, c=9.4258(3) \AA ; \beta=91.1279(17)^{\circ} ; V=574.61(3)$ 
$\AA^{3}, D_{x}=1.273 \mathrm{~g} \mathrm{~cm}^{-3}, 1778$ unique data $\left(2 \theta_{\max }=60.0^{\circ}\right), R=0.070$ [for 1655 reflections with $I>2.0 \sigma(I)] ; R w=0.156$ (all data), $S=1.03$.

Compound 30. $\mathrm{C}_{11} \mathrm{H}_{12} \mathrm{O}_{3}, M=192.21, T=150 \mathrm{~K}$, monoclinic, space group $P 2_{1}, Z=2$, $a=8.4949(4) \AA, b=5.9702(2) \AA, c=9.3840(4) \AA ; \beta=95.383(4)^{\circ} ; V=473.82(3) \AA^{3}$, $D_{x}=1.347 \mathrm{~g} \mathrm{~cm}^{-3}, 1032$ unique data $\left(2 \theta_{\max }=144.8^{\circ}\right), R=0.040$ [for 998 reflections with $I>2.0 \sigma(I)] ; R w=0.130$ (all data), $S=1.03$.

Compound 32. $\mathrm{C}_{10} \mathrm{H}_{12} \mathrm{O}_{2}, M=164.20, T=150 \mathrm{~K}$, orthorhombic, space group $P 2{ }_{1} 2_{1} 2_{1}$, $Z=4, a=7.8054(1) \AA, b=9.7920(1) \AA, c=11.0580(1) \AA ; V=845.17(2) \AA^{3}, D_{x}=$ $1.290 \mathrm{~g} \mathrm{~cm}^{-3}$, 1677 unique data $\left(2 \theta_{\max }=144.6^{\circ}\right), R=0.024$ [for 1645 reflections with $I>2.0 \sigma(I)] ; R w=0.057$ (all data), $S=1.01$.

Compound 34. $\mathrm{C}_{11} \mathrm{H}_{12} \mathrm{O}_{3}, M=192.21, T=150 \mathrm{~K}$, monoclinic, space group $P 2{ }_{1}, Z=2$, $a=6.9303(2) \AA, b=7.7696(2) \AA, c=8.6272(2) \AA ; \beta=92.403(2)^{\circ} ; V=464.13(2) \AA^{3}$, $D_{x}=1.375 \mathrm{~g} \mathrm{~cm}^{-3}, 993$ unique data $\left(2 \theta_{\max }=145.2^{\circ}\right), R=0.032$ [for 980 reflections with $I>2.0 \sigma(I)] ; R w=0.117$ (all data), $S=1.04$.

Compound 35. $\mathrm{C}_{13} \mathrm{H}_{16} \mathrm{O}_{3}, M=220.27, T=150 \mathrm{~K}$, orthorhombic, space group $P 2{ }_{1} 2_{1} 2_{1}$, $Z=4, a=7.3877(1) \AA, b=7.8178(1) \AA, c=19.8494(2) \AA ; V=1146.41(2) \AA^{3}, D_{x}=$ $1.276 \mathrm{~g} \mathrm{~cm}^{-3}$, 2267 unique data $\left(2 \theta_{\max }=144.8^{\circ}\right), R=0.045$ [for 2238 reflections with $I>2.0 \sigma(I)] ; R w=0.056$ (all data), $S=1.00$.

Compound 36. $\mathrm{C}_{11} \mathrm{H}_{12} \mathrm{O}_{3}, M=192.21, T=150 \mathrm{~K}$, orthorhombic, space group $P 2{ }_{1} 2_{1} 2_{1}$, $Z=4, a=7.4666(2) \AA, b=12.0831(5) \AA, c=10.8645(4) \AA ; V=980.19(6) \AA^{3}, D_{x}=$ $1.302 \mathrm{~g} \mathrm{~cm}^{-3}, 1491$ unique data $\left(2 \theta_{\max }=58.4^{\circ}\right), R=0.042$ [for 1303 reflections with $I$ $>2.0 \sigma(I)] ; R w=0.108$ (all data), $S=1.00$.

Compound 38. $\mathrm{C}_{10} \mathrm{H}_{12} \mathrm{O}_{2}, M=164.20, T=150 \mathrm{~K}$, hexagonal, space group $P 65, Z=6$, $a=14.7787(4) \AA, c=7.6213(2) \AA ; V=1441.56(7) \AA^{3}, D_{x}=1.135 \mathrm{~g} \mathrm{~cm}^{-3}, 1379$ 
unique data $\left(2 \theta_{\max }=58.8^{\circ}\right), R=0.025$ [for 1283 reflections with $\left.I>2.0 \sigma(I)\right] ; R w=$ 0.111 (all data), $S=1.03$.

Compound 41. $\mathrm{C}_{17} \mathrm{H}_{26} \mathrm{O}_{3}, M=278.39, T=150 \mathrm{~K}$, orthorhombic, space group $P 2{ }_{1} 2_{1} 2_{1}$, $Z=4, a=6.26451(4) \AA, b=12.09608(9) \AA, c=20.26568(14) \AA ; V=1535.65(2) \AA^{3}$, $D_{x}=1.204 \mathrm{~g} \mathrm{~cm}^{-3}, 3025$ unique data $\left(2 \theta_{\max }=144.6^{\circ}\right), R=0.032$ [for 2974 reflections with $I>2.0 \sigma(I)] ; R w=0.062$ (all data), $S=1.00$.

Compound 44. $\mathrm{C}_{16} \mathrm{H}_{22} \mathrm{O}_{4}, M=278.35, T=150 \mathrm{~K}$, orthorhombic, space group $P 2{ }_{1} 2_{1} 2_{1}$, $Z=4, a=6.4307(1) \AA, b=8.7685(1) \AA, c=26.1929(2) \AA ; V=1476.95(3) \AA^{3}, D_{x}=$ $1.252 \mathrm{~g} \mathrm{~cm}^{-3}, 2913$ unique data $\left(2 \theta_{\max }=144.8^{\circ}\right), R=0.030$ [for 2859 reflections with $I>2.0 \sigma(I)] ; R w=0.063$ (all data), $S=1.00$.

Compound 45. $\mathrm{C}_{18} \mathrm{H}_{24} \mathrm{O}_{5}, M=320.39, T=150 \mathrm{~K}$, monoclinic, space group $P 2_{1}, Z=2$, $a=7.4300(3) \AA, b=9.7667(3) \AA, c=12.1277(3) \AA ; \beta=92.279(3)^{\circ} ; V=879.37(5)$ $\AA^{3}, D_{x}=1.210 \mathrm{~g} \mathrm{~cm}^{-3}, 2377$ unique data $\left(2 \theta_{\max }=58.6^{\circ}\right), R=0.030$ [for 2242 reflections with $I>2.0 \sigma(I)] ; R w=0.093$ (all data), $S=1.02$.

Compound 47. $\mathrm{C}_{22} \mathrm{H}_{36} \mathrm{O}_{4} \mathrm{Si}, M=392.61, T=150 \mathrm{~K}$, orthorhombic, space group $P 2{ }_{1} 2_{1} 2, \quad Z=4, a=25.6494(7) \AA, b=14.1949(4) \AA, c=6.2343(2) \AA ; V=$ 2269.85(11) $\AA^{3}, D_{x}=1.149 \mathrm{~g} \mathrm{~cm}^{-3}, 5914$ unique data $\left(2 \theta_{\max }=58.8^{\circ}\right), R=0.045$ [for 5443 reflections with $I>2.0 \sigma(I)] ; R w=0.102$ (all data), $S=1.04$.

Compound 48. $\mathrm{C}_{23} \mathrm{H}_{26} \mathrm{O}_{5}, M=382.46, T=150 \mathrm{~K}$, orthorhombic, space group $P 2{ }_{1}{ }_{1}{ }_{2}$, $Z=4, a=19.9195(6) \AA, b=11.6626(3) \AA, c=8.7386(2) \AA ; V=2030.09(9) \AA^{3}, D_{x}=$ $1.251 \mathrm{~g} \mathrm{~cm}^{-3}, 2980$ unique data $\left(2 \theta_{\max }=58.6^{\circ}\right), R=0.037$ [for 2759 reflections with $I$ $>2.0 \sigma(I)] ; R w=0.101$ (all data), $S=1.03$.

Compound 53. $\mathrm{C}_{23} \mathrm{H}_{26} \mathrm{O}_{5}, M=382.46, T=150 \mathrm{~K}$, orthorhombic, space group $P 2{ }_{1} 2_{1} 2_{1}$, $Z=4, a=8.9344(2) \AA, b=15.4099(3) \AA, c=14.9268(3) \AA ; V=2055.09(7) \AA^{3}, D_{x}=$ 
$1.236 \mathrm{~g} \mathrm{~cm}^{-3}$, 3065 unique data $\left(2 \theta_{\max }=59.0^{\circ}\right), R=0.036$ [for 2868 reflections with $I$ $>2.0 \sigma(I)] ; R w=0.112$ (all data), $S=1.04$.

Structure Determination. Images for compound 27, 36, 38, 45, 47, 48 and 53 were measured on a diffractometer (Mo K $\alpha$, graphite monochromator, $\lambda=0.71073 \AA$ ) fitted with an area detector and the data extracted using the DENZO/Scalepack package. ${ }^{15}$ Images for compounds $8,9,10,13,23,30,32,34,35,41$ and 44 were measured on a diffractometer ( $\mathrm{Cu} \mathrm{K} \alpha$, mirror monochromator, $\lambda=1.54184 \AA$ ) fitted with an area detector and the data extracted using the CrysAlis package. ${ }^{16}$ The structure solutions for all six compounds were solved by direct methods (SIR92) ${ }^{17}$ then refined using the CRYSTALS program package. ${ }^{18}$ Atomic coordinates, bond lengths and angles, and displacement parameters have been deposited at the Cambridge Crystallographic Data Centre (CCDC nos. 1545223, 154224, 1545225, 1545226, 1545227, 1545228, 1545229, 1545230, 1545231, 1545232, 1545233, 1545234, 1545235, 1545236, 1545237, 1545238, 1545239 and 1545240). These data can be obtained free-of-charge via www.ccdc.cam.ac.uk/data_request/cif, by emailing data_request@ccdc.cam.ac.uk, or by contacting The Cambridge Crystallographic Data Centre, 12 Union Road, Cambridge CB2 1EZ, UK; fax: +44 1223336033.

\section{ASSOCIATED CONTENT}

\section{Supporting Information}

Cifs and Anisotropic Displacement Ellipsoid Plots from the Single-crystal X-ray Analyses of Compounds 8, 9, 10, 13, 23, 27, 30, 32, 34, 35, 36, 38, 41, 44, 45, 47, 48 and 53. ${ }^{1} \mathrm{H}$ and ${ }^{13} \mathrm{C}$ NMR spectra of compounds 8-39 and 42-58. This material is available free-of-charge via the Internet at http://pubs.acs.org. 


\section{AUTHOR INFORMATION}

\section{Corresponding Author}

*Tel: +61-2-6125-8202. Fax: +61-2-6125-8114. E-mail: Martin.Banwell@anu.edu.au

\section{Notes}

The authors declare no competing financial interest.

\section{ACKNOWLEDGMENTS}

We thank the Australian Research Council and the Institute of Advanced Studies for financial support. QY is the grateful recipient of a CSC PhD Scholarship provided by the Government of the People’s Republic of China.

\section{REFERENCES AND NOTES}

1. For a relevant and recent review see Banwell, M. G.; Bon, D. J.-Y. D. Applications of the Di- $\pi$-Methane and Related Rearrangement Reactions in Chemical Synthesis. In Molecular Rearrangements in Organic Synthesis: Rojas, C. M., Ed.; Wiley, Hobokjen, NJ, 2015; Chapter 9, pp 261-288.

2. Luo, S.-Y.; Jang, Y.-J.; Liu, J.-Y.; Chu, C.-S.; Liao, C.-C.; Hung, S.-C. Angew. Chem. Int. Ed. 2008, 47, 8082 and references cited therein.

3. For reviews on cis-1,2-dihydrocatechols see: (a) Johnson, R. A. Org. React. 2004, 63, 117; (b) Hudlicky, T.; Reed, J. W. Synlett. 2009, 685; (c) Lewis, S. E. Chem. Commun. 2014, 50, 2821; (d) Banwell, M. G.; Bolte, B.; Buckler, J. N.; Chang, E. L.; Lan, P.; Taher, E. S.; White, L. V.; Willis, A. C. J. \& Proc. Royal Soc. New South Wales, 2016, 149, 34.

4. (a) Banwell, M. G.; Edwards, A. J.; Harfoot, G. J.; Jolliffe, K. A. Tetrahedron, 2004, 60, 535; (b) Banwell, M. G.; Austin, K. A. B.; Willis, A. C. Tetrahedron, 
2007, 63, 6388; (c) Reekie, T. A.; Austin, K. A. B.; Banwell, M. G.; Willis, A. C. Aust. J. Chem. 2008, 61, 94; (d) Bon, D. J.-Y. D.; Banwell, M. G.; Willis, A. C. Tetrahedron, 2010, 66, 7807; (e) Bon, D. J.-Y. D.; Banwell, M. G.; Cade, I. A.; Willis, A. C. Tetrahedron, 2011, 67, 8348; (f) Bon, D. J.-Y. D.; Banwell, M. G.; Ward, J. S.; Willis, A. C. Tetrahedron, 2013, 69, 1363.

5. (a) Schwartz, B. D.; Matoušová, E.; White, R.; Banwell, M. G.; Willis, A. C. Org. Lett. 2013, 15, 4580; (b) Chang, E. L.; Bolte, B.; Lan, P.; Willis, A. C.; Banwell, M. G. J. Org. Chem. 2016, 81, 2078.

6. Lan, P.; Banwell, M. G.; Willis, A. C. Org. Lett. 2015, 17, 166.

7. Yan, Q.; Banwell, M. G.; Coote, M. L.; Lee, R.; Willis, A. C. Chem. Asian J. 2017, DOI: 10.1002/asia.201700456.

8. Banwell, M. G.; Chun, C.; Edwards, A. J.; Vögtle, M. M. Aust. J. Chem. 2003, $56,861$.

9. Ireland, R. E.; Liu, L. J. Org. Chem. 1993, 58, 2899.

10. This protocol is based on one first described by Ma and Bobbitt: Ma, Z.; Bobbitt, J. M. J. Org. Chem. 1991, 56, 6110. For examples of related oxidations see Refs. 4b-d.

11. Ravelli, D.; Fagnoni, M. Photochemistry of Phosphate and Sulfonate Esters In CRC Handbook of Organic Photochemistry ( $3^{\text {rd }}$ Edition): Griesbeck, A.; Oelgemoller, M.; Ghetti, F., Ed.; CRC Press, Boca Raton, FL, 2012; Chapter 17, pp 393-417.

12. Still, W. C.; Kahn, M.; Mitra, A. J. Org. Chem. 1978, 43, 2923.

13. Pangborn, A. B.; Giardello, M. A.; Grubbs, R. H.; Rosen, R. K.; Timmers, F. J. Organometallics 1996, 15, 1518.

14. Foley, D. A.; O’Leary, P.; Buckley, N. R.; Lawrence, S. E.; Maguire, A. R. 
Tetrahedron, 2013, 69, 1778.

15. DENZO-SMN. Otwinowski, Z.; Minor, W. Processing of X-ray diffraction data collected in oscillation mode. In Methods in Enzymology, Volume 276: Macromolecular Crystallography, Part A; C. W. Carter Jr. and R. M. Sweet, Eds.; Academic Press: New York, 1997; pp. 307-326.

16. CrysAlis PRO Version 1.171.37.35h (release 09-02-2015 CrysAlis171.NET) (compiled Feb 9 2015,16:26:32) Agilent Technologies: Oxfordshire, UK.

17. SIR92. Altomare, A.; Cascarano, G.; Giacovazzo, C.; Guagliardi, A.; Burla, M. C.; Polidori, G.; Camalli, M. J. Appl. Crystallogr. 1994, 27, 435.

18. Betteridge, P. W.; Carruthers, J. R.; Cooper, R. I.; Prout, K.; Watkin, D. J. J. Appl. Crystallogr. 2003, 36, 1487. 